\title{
Reconciling Event-Labeled Gene Trees with MUL-trees and Species Networks
}

\author{
Marc Hellmuth ${ }^{1,2}$, Katharina T. Huber ${ }^{3}$, and Vincent Moulton ${ }^{3}$ \\ ${ }^{1}$ Institute of Mathematics and Computer Science, University of Greifswald, Walther-Rathenau-Strasse 47, \\ D-17487 Greifswald, Germany, Email: mhel lmuth@mailbox.org \\ ${ }^{2}$ Saarland University, Center for Bioinformatics, Building E 2.1, P.O. Box 151150, D-66041 Saarbrücken, \\ Germany \\ ${ }^{3}$ School of Computing Sciences, University of East Anglia, Norwich, UK, Email: K. Huber@uea .ac.uk and \\ v.moulton@uea.ac.uk
}

\begin{abstract}
Phylogenomics commonly aims to construct evolutionary trees from genomic sequence information. One way to approach this problem is to first estimate event-labeled gene trees (i.e., rooted trees whose non-leaf vertices are labeled by speciation or gene duplication events), and to then look for a species tree which can be reconciled with this tree through a reconciliation map between the trees. In practice, however, it can happen that there is no such map from a given event-labeled tree to any species tree. An important situation where this might arise is where the species evolution is better represented by a network instead of a tree. In this paper, we therefore consider the problem of reconciling event-labeled trees with species networks. In particular, we prove that any event-labeled gene tree can be reconciled with some network and that, under certain mild assumptions on the gene tree, the network can even be assumed to be multi-arc free. To prove this result, we show that we can always reconcile the gene tree with some multi-labeled (MUL-)tree, which can then be "folded up" to produce the desired reconciliation and network. In addition, we study the interplay between reconciliation maps from event-labeled gene trees to MUL-trees and networks. Our results could be useful for understanding how genomes have evolved after undergoing complex evolutionary events such as polyploidy.
\end{abstract}

Keywords: tree reconciliation; network reconciliation; gene evolution; species evolution; phylogenetic network; MUL tree; triples

\section{Introduction}

Phylogenomics aims to find plausible hypotheses about the evolutionary history of species based on genomic sequence information. Such hypotheses often take the form of an evolutionary tree whose leaves are labeled by the species in question, or a species tree. There are various ways to construct species trees from genomics data, many of which involve estimating the evolutionary history of the underlying genes, and then using the resulting gene trees to construct a species tree [45].

A recent example of such an approach relies on using event-labeled gene trees [25, 26, 28]. These are trees in which the leaves correspond to the genes, inner vertices to ancestral genes, and the label of an inner vertex corresponds to the divergence event that led to the offspring. Such events include speciation and duplication events which correspond to genes that are orthologous 
or paralogous $[16,17]$, respectively, that is, they diverged after a speciation event or from one another within a species after a duplication event. To estimate event-labeled gene trees, sequence similarities and synteny information is first used to determine which genes are orthologous [1$3,5,38,39,49,54,57]$, and the trees are then estimated from the resulting orthology relations $[8,10,11,24,34,36]$ using an underlying "cograph"-structure $[24,27]$.

Once an event-labeled gene tree has been estimated, the task then becomes finding a species tree which accommodates the gene history. This is essentially done by looking for a mapping (a so-called reconciliation) from the gene tree into some species tree which respects ancestral relationships. Note that the problem of reconciling gene trees with species trees has been studied for some time (mainly for non-event-labeled gene trees) [9, 12-15, 19, 20, 33, 37, 41, 42, 44, 46, $51-53,56,58]$. However, one issue with this overall process for constructing species trees is that it may not be possible to reconcile an event-labeled gene tree with any species tree [26, 28]. This may be because of inaccuracies in estimating the trees, but a more fundamental problem can arise due to the fact that the species tree is not an appropriate way to represent the evolution of the species in question.

More specifically, it is well-known that species can come together with each other to form new ones through processes such as hybridization or recombination [18] (a process sometimes called reticulate evolution). In this case it can be more appropriate to represent species evolution using a species network instead of a species tree [4]. Interestingly, as we shall see, it may be possible to reconcile an event-labeled gene tree with a species network even though it is not possible to reconcile it with any species tree. The aim of this paper is to better understand why this is the case, and to develop new theory and techniques for reconciling event-labeled gene trees with species networks. Note that some work has appeared on the problem of reconciling non event-labeled gene trees with given species networks $[48,55]$. However, to our best knowledge, the problem of reconciling event-labeled gene trees with unknown species networks has not yet been considered.

The rest of this paper is organized as follows. We start with basic definitions concerning phylogenetic trees and networks in Section 2. In Section 3, we then give a new definition of a reconciliation map between arbitrary, possibly non-binary, event-labeled gene trees and species networks, called TreeNet-reconciliation maps. We show that this definition is a natural generalization of the reconciliation maps defined in [55], between binary gene trees and binary species networks. In Section 4, we also prove that TreeNet-reconciliation maps are equivalent to reconciliation maps between trees $[12,26,28]$ in case the considered species network is a tree. Continuing with this theme, we also examine the problem of determining when there exists a TreeNet-reconciliation from an event-labeled tree to some species tree in Section 5.

In Section 7 we show that for every event-labeled gene tree $T$, there always exists a TreeNetreconciliation map from $T$ to some species network $N$. To prove this result we employ the concept of so-called MUL-trees [6, 7, 23, 29, 31, 40, 47]. Our proof essentially relies on first defining a reconciliation map between an event-labeled tree and a multiple-labeled tree, or $M U L$-tree. Once we have a reconciliation between $T$ and $M$, we then apply a so-called folding map [30] to $M$ to produce a species network $N$ (as explained in Section 6), which induces the desired TreeNetreconciliation map between $T$ and $N$. This whole process allows us to determine the TreeNetreconciliation map and network for the gene tree in polynomial-time in the number of genes.

The use of folding maps can result in species networks which contain multi-arcs, which may be somewhat unrealistic for applications. In Section 8 we therefore strengthen the main result in Section 7, showing that given an event-labeled gene tree that satisfies a simple biological constraint on its speciation vertices (cf. Def. 8), there always exists a TreeNet-reconciliation map from $T$ to some multi-arc free species network. Finally, in Section 9, we study the interplay between TreeNet-reconciliation maps from event-labeled gene trees to MUL-trees and networks. This topic has recently become of interest in the phylogenomics literature, where it has been applied to understand polyploid evolution [21]. In particular, in this work the authors use the interplay between MUL-trees and phylogenetic networks to try to distinguish between different types of 
ploidy events. We conclude with a discussion of some open problems in Section 9.

\section{Preliminaries}

\section{Phylogenetic Networks and Trees}

In what follows, the set $X$ always denotes a finite set of size at least two. Moreover, $\mathbb{G}$ and $\mathbb{S}$ will denote a set of genes and species, respectively.

In general, in this paper a directed graph or digraph, for short, may contain multi-arcs, that is, two or more arcs that connect two vertices. We consider rooted, not necessarily binary phylogenetic trees and networks, called trees or networks for short (see e.g. [50] for an overview of phylogenetic trees and networks). To be more precise:

Definition 1. A network $N=(V, E)$ on $X$ is a directed acyclic graph $(D A G)$ with leaf set $L(N)=X$, multi-arcs allowed that satisfies the following properties

(N1) There is a single root $\rho_{N}$ with indegree 0 and outdegree 1 such that its unique child has indegree 1 and outdegree at least 2;

(N2) $x \in X$ if and only if $x$ is an outdegree-0 and indegree-1 vertex.

(N3) Each vertex $v \in V^{0}:=V \backslash X$ with $v \neq \rho_{N}$ has either

indegree 1 and outdegree greater than 1 (tree vertex) or

indegree greater than 1 and outdegree 1 (hybrid vertex)

If $X$ is a set of genes $\mathbb{G}$ (resp. species $\mathbb{S}$ ), then $N$ is called a gene (resp. species) network. A network that has no multi-arcs is called multi-arc free and a multi-arc free network that has no hybrid vertices is called a (phylogenetic) tree.

We call a tree reduced if it is obtained from a phylogenetic tree $T$ by removing the root $\rho_{T}$ and its unique incident arc from $T$. Hence, the root of a reduced tree always has degree greater or equal to 2 .

Note, Property (N1) differs slightly from the usual notion as used e.g. in [48, 55], where $\rho_{N}$ has indegree 0 and outdegree 2 . We need this extra condition since our notion of a reconciliation map between a gene tree and a species network allows for the possibility that an event occurred before the first speciation event in the network.

Now, suppose that $N=(V, E)$ is a phylogenetic network with leaf set $X$. All vertices within $V \backslash X$ are called inner vertices. Given an $\operatorname{arc} e=(x, y)$ in $N, y$ is the head of $e$, denoted by $h_{N}(e)$, and $x$ is the tail of $e$, denoted by $t_{N}(e)$. In this case, we also say that $x$ is the parent $\operatorname{par}(y)$ of $y$. In addition, for any DAG $G=(V, E)$ and any vertex $v \in V$ that has a unique incoming arc, we denote this arc by $e^{\nu}$.

A directed path from a vertex $x_{1}$ to another vertex $x_{l}$ in $N$ is a non-empty sequence $P=$ $\left(x_{1}, \ldots, x_{l}\right)$ of pairwise disjoint vertices such that $\left(x_{i}, x_{i+1}\right) \in E, 1 \leq i \leq l-1$. We often denote the directed path $P=\left(x_{1}, \ldots, x_{l}\right)$ by $P\left(x_{1}, x_{l}\right)$ and also write $P^{\prime}=P v$ (resp. $P^{\prime}=P e$ with $\left.e=\left(x_{l}, v\right)\right)$ for the directed path $P^{\prime}=\left(x_{1}, \ldots, x_{l}, v\right)$ that is obtained from the directed path $P\left(x_{1}, x_{l}\right)$ by adding the vertex $v$. Moreover, for $x \in V$ and $e \in E$, we define the directed path from $x$ to $e$ in $N$ as the directed path $P$ from $x$ to the head $h_{N}(e)$, if $P$ exists. Thus, the directed path from $x$ to the arc $e=(x, y)$ coincides with $e$.

Let $u, v \in V$. Then, a vertex $v \in V$ is called a descendant of $u$ (in symbols, $v \preceq_{N} u$ ), if there is a directed path (possibly reduced to a single vertex) in $N$ from $u$ to $v$. In this case, we also call $u$ an ancestor of $v$, denoted by $u \succeq_{N} v$. If $u \preceq_{N} v$ or $v \preceq_{N} u$ then $u$ and $v$ are comparable and otherwise, incomparable. Moreover, if $v \preceq_{N} u$ and $u \neq v$, then $v$ is below $u$ and $u$ above $v$. Note, since $N$ is a DAG, an arc $e=(u, v)$ always implies that $u \succ_{N} v$. For a vertex $x \in V$, we write $L_{N}(x):=\left\{y \in X \mid y \preceq_{N} x\right\}$ for the set of leaves in $X$ that are below or equal to $x$. 
For our discussion below we need to extend the definition of $\preceq_{N}$ to $V \cup E$. More precisely, for the arc $e=(u, v) \in E$ we put $x \prec_{N} e$ if $x \preceq_{N} v$ and $e \prec_{N} x$ if $u \preceq_{N} x$. In this case, the vertex $x$ and the arc $e$ are comparable, and incomparable otherwise. If $e=(u, v)$ and $f=(a, b)$ are $\operatorname{arcs}$ in $N$, then we define $e \preceq_{N} f$ to hold if $v \prec u \preceq b \prec a$ or $e=f$. In this case, the arcs $e$ and $f$ are also comparable, and incomparable otherwise.

We say that a network $N^{\prime}$ is a subdivision of $N$, if $N^{\prime}$ can be obtained from $N$ by replacing arcs $(u, v)$ of $N$ by directed paths from $u$ to $v$. Hence, a network $N$ is also a subdivision of itself. Let $W \subseteq V$. The subgraph of $N$ with vertex set $W$ that contains all $\operatorname{arcs}(x, y) \in E$ for which $x, y \in W$ is called induced subgraph of $N$ and is denoted by $N[W]$.

\section{Common Ancestors}

For a non-empty subset of leaves $A \subseteq X$ of a phylogenetic tree $T=(V, E)$ on $X$, we define $\operatorname{lca}_{T}(A)$, the least common ancestor of $A$, to be the unique $\preceq_{T}$-minimal vertex of $T$ that is an ancestor of every vertex in $A$. In case $A=\{x, y\}$, we put lca $T(x, y):=\operatorname{lca}_{T}(\{x, y\})$ and if $A=\{x, y, z\}$, we put $\operatorname{lca}_{T}(x, y, z):=\operatorname{lca}_{T}(\{x, y, z\})$. If $e, f \in E$ and $x \in V$, then we define $\operatorname{lca}_{T}(x, e):=\operatorname{lca}_{T}\left(x, t_{T}(e)\right)$ and $\operatorname{lca}_{T}(e, f):=\operatorname{lca}_{T}\left(t_{T}(e), t_{T}(f)\right)$.

Given a tree vertex $z$ in a network $N=(V, E)$, two (not necessarily disjoint) directed paths in $N$ that start from $z$ are said to be separated (by $z$ ) if each path contains a different child of $z$. Given (not necessarily distinct) $x, y \in V$, we denote by $Q_{N}(x, y)$ the set of vertices $z$ of $N$ such that there exists a directed path $P(z, x)$ and a directed path $P(z, y)$ such that $P(z, x)$ and $P(z, y)$ are separated by $z$. Note, $Q_{N}(x, y)=Q_{N}(y, x)$. If $z \in Q_{N}(x, y)$, then we also say that $x$ and $y$ are separated by $z$. By way of example, we have $Q_{N}(x, x)=\{a, w\}$ and $Q_{N}(x, y)=\{b, w\}$ for the network $N$ in Fig. 4 (right). We generalize the latter also to arcs and put $Q_{N}(x, e):=Q_{N}\left(x, h_{N}(e)\right)$ and $Q_{N}(e, f):=Q_{N}\left(h_{N}(f), h_{N}(e)\right)$ for all arcs $e, f \in E$.

The definition of $Q_{N}(x, y)$ was presented in [55], to generalize the concept of least common ancestors in binary trees to binary networks. We now generalize this definition to cope with networks that are not necessarily binary. More specifically, given a collection $x_{1} \ldots, x_{k}$ of not necessarily distinct vertices in $N$, we let

$$
Q_{N}^{2}\left(x_{1}, \ldots, x_{k}\right):=\bigcup_{1 \leq i \leq j \leq k} Q_{N}\left(x_{i}, x_{j}\right)
$$

be the set of vertices that separate any $x_{i}$ and $x_{j}, 1 \leq i \leq j \leq k$.

\section{Event-labeled Gene Trees}

An event-labeled gene tree $(T ; t, \sigma)$ on $\mathbb{G}$ is a reduced tree $T=(V, E)$ with leaf set $\mathbb{G}$ (a set of genes) a labeling map of events $t: V^{0} \rightarrow\{\mathfrak{s}, \mathfrak{d}\}$, and a surjective map $\sigma: \mathbb{G} \rightarrow \mathbb{S}$, called genespecies map, that assigns to each gene $g \in \mathbb{G}$ the species $\sigma(g)$ that contains $g$.

Note, the main difference between the structure of an event-labeled gene tree $(T ; t, \sigma)$ and a phylogenetic tree $T^{\prime}$ as in Definition 1 is that the root of $T$ has at least two children, while the root of $T^{\prime}$ has exactly one child. The events $\mathfrak{s}$ and $\mathfrak{d}$ are called speciation and duplication, respectively. Moreover, a vertex $v \in V^{0}$ with $t(v)=\mathfrak{s}$ is called a speciation vertex, and otherwise, a duplication vertex. In all figures that contain event-labeled gene trees, the events $\mathfrak{s}$ and $\mathfrak{d}$ are represented as $\bullet$ and $\square$, respectively.

In addition, for a subset $W \subseteq \mathbb{G}$, we put $\sigma(W):=\{\sigma(w) \mid w \in W\}$.

In what follows, we will always assume that for an event-labeled gene tree $(T ; t, \sigma)$ on $\mathbb{G}$, $|\sigma(\mathbb{G})|>1$ holds, i.e., the genes in $\mathbb{G}$ are from at least two distinct species.

\section{MUL-trees}

Informally speaking, a MUL-tree (MUltiply-Labelled-tree) is a phylogenetic tree $M$ (cf. Definition 1) where each leaf in $M$ is labeled by an element in $\mathbb{S}$, but where different leaves may have 
the same label. More precisely, a MUL-tree $($ on $\mathbb{S})$ is a pair $(M, \chi)$ where $M$ is a phylogenetic tree and $\chi: \mathbb{S} \rightarrow 2^{L(M)}-\{\emptyset\}$ is a map such that for all $x, y \in \mathbb{S}$ distinct, $\chi(x) \cap \chi(y)=\emptyset$ and for all $l \in L(M)$ there exists some $x \in \mathbb{S}$ with $l \in \chi(x)$. Thus, in a MUL-tree $M$ the labeling of the leaf set $L(M)$ of $(M, \chi)$ is the multiset whose underlying set is $\mathbb{S}$. Note that our definition of a MUL-tree is equivalent to the definition given in [30, Section 2.2], except that in the MUL-tree defined here we have an "extra" arc which is adjacent to the root (so we get a root with outdegree 1). As with networks, this extra arc is required to accommodate events in reconciliations which occur before the first speciation.

If we allow $M$ to contain vertices with indegree 1 and outdegree 1 as well, then we call $M$ a pseudo MUL-tree (so, in this setting, any MUL-tree is also considered as a pseudo MUL-tree). For a pseudo MUL-tree $M=(D, U)$, we also define

$$
D^{1}=\{v \in D \mid v \text { has in- and outdegree one in } M\} .
$$

We say that two (pseudo) MUL-trees $\left(M_{1}, \chi_{1}\right)$ and $\left(M_{2}, \chi_{2}\right)$ on $X$ are isomorphic if there is a digraph isomorphism $\varphi: V\left(M_{1}\right) \rightarrow V\left(M_{2}\right)$ such that, for all $x \in X$ and $v \in V\left(M_{1}\right)$, we have $v \in$ $\chi_{1}(x)$ if and only if $\varphi(v) \in \chi_{2}(x)$. In addition,we say that a pseudo-MUL-tree $\left(M^{\prime}, \chi\right)$ is a simple subdivision of the MUL-tree $(M=(D, U), \chi)$ if $M^{\prime}$ is a subdivision of $M$ that is obtained from $M$ by replacing every arc $e=(u, v)$ of $M$, where $v$ is a leaf of $M$ such that $v \in \chi(x)$ with $x \in \mathbb{S}$ and $|\chi(x)| \geq 2$ by the path $\left(u, v_{e}, v\right)$ in $M^{\prime}$, where $v_{e} \notin D$. The latter, implies in particular that $L(M)=L\left(M^{\prime}\right)$ and hence, the map $\chi$ for $M^{\prime}$ is well-defined.

\section{Reconciliation Maps to Networks}

In [55], a notion of a reconciliation map (which we shall call a biTreeNet-reconciliation map) from a phylogenetic tree to a network was presented (see Definition 3). This map, however, assumes that the event labels on the tree are unknown, while a species network is given. Moreover, the biTreeNet-reconciliation map "axioms" explicitly refer to binary gene trees and binary species networks. In practice, when gene trees are obtained from e.g. orthology-data, we cannot hope to obtain fully resolved (i.e., binary) gene trees. To overcome this problem and therefore also be able to study reconciliations between non-binary gene trees and (possibly unknown) non-binary networks we next introduce the novel concept of a TreeNet-reconciliation map. That TreeNetreconciliation maps indeed generalize biTreeNet-reconciliation maps is shown in Proposition 1. Furthermore, TreeNet-reconciliation maps provide a natural generalization of the framework as used in $[26,28,43]$ for the reconciliation of trees, which is shown in Section 4.

Definition 2 (TreeNet-reconciliation map). Suppose that $\mathbb{S}$ is a set of species, that $N=(W, F)$ is a network on $\mathbb{S}$, and that $(T=(V, E) ; t, \sigma)$ is an event-labeled gene tree on $\mathbb{G}$. Then we say that $N$ is a (species) network for $(T ; t, \sigma)$ if there is a map $\mu: V \rightarrow W \cup F$ such that, for all $x \in V$ :

(R1) Leaf Constraint. If $x \in \mathbb{G}$ then $\mu(x)=\sigma(x)$.

(R2) Event Constraint.

(i) If $t(x)=\mathfrak{s}$ and $x$ has children $x_{1}, \ldots, x_{k}, k \geq 2$, then $\mu(x) \in Q_{N}^{2}\left(\mu\left(x_{1}\right), \ldots, \mu\left(x_{k}\right)\right)$.

(ii) If $t(x)=\mathfrak{d}$, then $\mu(x) \in F$.

(R3) Ancestor Constraint.

Suppose $x, y \in V$ with $x \prec_{T} y$.

(i) If $t(x)=t(y)=\mathfrak{d}$, then $\mu(x) \preceq_{N} \mu(y)$,

(ii) Otherwise, i.e., at least one of $t(x)$ and $t(y)$ is a speciation, $\mu(x) \prec_{N} \mu(y)$.

We call $\mu$ a TreeNet-reconciliation map from $(T ; t, \sigma)$ to $N$. 
Property (R1) ensures that each leaf of $T$, i.e., each gene in $\mathbb{G}$, is mapped to the species in which it resides. Property (R2.i) implies the weaker property that $\mu(x) \in W^{0}$. Moreover, Property (R2.i) ensures that each speciation vertex $x$ of $T$ is mapped to a vertex in $N$ that separates at least two of the images $\mu\left(x_{i}\right)$ and $\mu\left(x_{j}\right)$ of the children $x_{1}, \ldots, x_{k}, k \geq 2$, of $x$. We emphasize that, even in the case $\mu\left(x_{1}\right)=\mu\left(x_{2}\right)=\cdots=\mu\left(x_{k}\right)$, it is possible that $Q_{N}^{2}\left(\mu\left(x_{1}\right), \ldots, \mu\left(x_{k}\right)\right) \neq \emptyset$, since $N$ may contain distinct directed paths from $\mu(x)$ to $\mu\left(x_{1}\right)$ that separate $\mu\left(x_{1}\right)$. Property (R2.ii) ensures that each duplication vertex of $T$ is mapped to an $\operatorname{arc}$ in $N$. Property (R3) implies that the ancestor relationships in $T$ are preserved under $\mu$. Note, however, that two different duplication vertices might be mapped to the same arc of $N$ under $\mu$.

We now show that for the special case that both the gene tree and the species network are binary, Definition 2 is a natural generalization of biTreeNet-reconciliation maps as defined in [55]. We begin by recalling the definition of this map (using our notation). Note, in this definition the symbols $\mathfrak{s}$ and $\mathfrak{d}$ still denote speciation and duplication events, respectively. For technical reasons, an additional symbol $\mathfrak{c}$ is used to annotate leaves.

Definition 3 (biTreeNet-reconciliation map [55]). Let $\sigma: \mathbb{G} \rightarrow \mathbb{S}$ be a gene-species map. A biTreeNet-reconciliation map $\alpha=\left(\alpha_{1}, \alpha_{2}\right)$ from a binary gene tree $T=(V, E)$ on $\mathbb{G}$ to a binary network $N=(W, F)$ on $\mathbb{S}$ is a pair of maps $\alpha_{1}: V \rightarrow W$ and $\alpha_{2}: V \rightarrow\{\mathfrak{s}, \mathfrak{d}, \mathfrak{c}\}$ that assigns to each vertex $u \in V$ a pair $\left(\alpha_{1}(u), \alpha_{2}(u)\right)$ such that

(A1) $\alpha_{2}(u)=\mathfrak{c}$ if and only if $u \in \mathbb{G}, \alpha_{1}(u) \in L(N), \sigma(u)=\alpha_{1}(u)$,

(A2) for every $u \in V^{0}$ with child vertices $u_{1}$ and $u_{2}$,

$$
\text { if } \alpha_{2}(u)=\mathfrak{s} \text {, then } \alpha_{1}(u) \in Q_{N}\left(\alpha_{1}\left(u_{1}\right), \alpha_{1}\left(u_{2}\right)\right) \text {, and }
$$

(A3) for all $u, v \in V$ such that $v \prec_{T} u$,

$$
\text { if } \alpha_{2}(u)=\mathfrak{d} \text {, then } \alpha_{1}(v) \preceq_{N} \alpha_{1}(u) \text {. Otherwise, } \alpha_{1}(v) \prec_{N} \alpha_{1}(u) \text {. }
$$

In Definition 3, $\alpha_{2}$ plays the role of our event-labeling map. That is, if $\alpha_{2}$ is given, then putting $t^{\alpha}(u):=\alpha_{2}(u)$ for all $u \in V^{0}$ yields an event-labeled gene tree $\left(T ; t^{\alpha}, \sigma\right)$ on $\mathbb{G}$.

Proposition 1. If there is a biTreeNet-reconciliation map $\alpha$ from a binary gene tree $T$ on $\mathbb{G}$ to a binary network $N$ on $\mathbb{S}$, then there is a TreeNet-reconciliation map from $\left(T ; t^{\alpha}, \sigma\right)$ to $N$.

Proof. In what follows, we show that there is a TreeNet-reconciliation map $\mu$ from $\left(T ; t^{\alpha}, \sigma\right)$ to $N$, where $t^{\alpha}$ is constructed as described above.

Let $\alpha=\left(\alpha_{1}, \alpha_{2}\right)$ be a biTreeNet-reconciliation map from $T=(V, E)$ to $N=(W, F)$. We claim that $\mu: V \rightarrow W \cup F$ given by

$$
\mu(u)= \begin{cases}\alpha_{1}(u) & \text { if } t^{\alpha}(u) \in\{\mathfrak{s}, \mathfrak{c}\}, \\ e^{\alpha_{1}(u) \in F} & \text { if } t^{\alpha}(u)=\mathfrak{d} \text { and } \alpha_{1}(u) \text { is not a hybrid vertex }, \\ \left(\alpha_{1}(u), x\right) \in F & \text { otherwise, some } x \in W .\end{cases}
$$

is a TreeNet-reconciliation map from $\left(T ; t^{\alpha}, \sigma\right)$ to $N$. Note, in the second condition $e^{\alpha_{1}(u)}$ is welldefined, since $\alpha_{1}(u)$ must, in this case, be either the root $\rho_{N}$ or a tree vertex in $N$ and thus, there is only one incoming arc to $\alpha_{1}(u)$. Moreover, in the last condition, the vertex $x$ is uniquely defined, since $\alpha_{1}(u)$ is then a hybrid vertex and thus, there is only arc $\left(\alpha_{1}(u), x\right)$ in $N$.

Clearly, Property (A1) and the construction of $\mu$ immediately implies Property (R1).

We show now that Property (R3) is satisfied. Let $u, v \in V$ such that $v \prec_{T} u$. First assume that $t^{\alpha}(u)=\mathfrak{d}$. There are now two mutually exclusive cases: either $\alpha_{1}(u)$ is not a hybrid vertex and $\mu(u)$ is the $\operatorname{arc} e^{\alpha_{1}(u)}$ or $\alpha_{1}(u)$ is a hybrid vertex and $\mu(u)$ is the $\operatorname{arc}\left(\alpha_{1}(u), x\right)$, some $x \in$ $W$. If $t^{\alpha}(v)=\mathfrak{d}$, then, in both cases, Property (A3) and a straight-forward case analysis implies that $\mu(v) \preceq_{N} \mu(u)$. If $t^{\alpha}(v)=\mathfrak{s}$, then Property (A2) implies that $\mu(v)$ must be a tree vertex. By construction, if $\alpha_{1}(v)=\alpha_{1}(u)$, then $\mu(u)$ is mapped to the unique arc $e^{\mu(v)}$ and we obtain 
$\mu(v) \prec_{N} \mu(u)$. In particular, $\alpha_{1}(v) \preceq_{N} \alpha_{1}(u)$ implies that the $\operatorname{arc} e^{\mu(v)}$ is the "lowest" possible choice for $\mu(u)$ and thus, we always have $\mu(v) \prec_{N} \mu(u)$, in case $t^{\alpha}(v)=\mathfrak{s}$.

Now assume that $t^{\alpha}(u)=\mathfrak{s}$. Then, $\mu(u)=\alpha_{1}(u)$. In particular, Property (A1) implies $\mu(u) \in$ $V^{0}$. Property (A2) and the construction of $\mu$ implies that $\mu(u)$ must be a tree vertex. If $t^{\alpha}(v)=\mathfrak{s}$ or $v \in \mathbb{G}$, then $\alpha_{1}$ and $\mu$ coincide on $u$, resp., on $v$. Hence, Property (A3) implies $\mu(v) \prec_{N} \mu(u)$. Thus, assume that $t^{\alpha}(v)=\mathfrak{d}$. The fact that $\mu(u)$ is not a hybrid vertex together with Property (A3) and the construction of $\mu$ implies that $\mu(v)=(x, y) \prec_{N} x \preceq_{N} \alpha_{1}(u)=\mu(v)$. In summary, Property (R3) is satisfied.

We continue by showing that Property (R2) is satisfied. By construction of $\mu$, Property (R2.ii) is satisfied. For Property (R2.i), let $u$ be a vertex in $T$ with $t^{\alpha}(u)=\mathfrak{s}$. Thus, $\mu(u)=\alpha_{1}(u)$. Since $T$ is binary, $u$ has exactly two children $u_{1}$ and $u_{2}$. Property (A2) implies $\mu(u)=\alpha_{1}(u) \in$ $Q_{N}\left(\alpha_{1}\left(u_{1}\right), \alpha_{1}\left(u_{2}\right)\right)$. Thus, $\mu(u)$ must be a tree vertex. In particular, there are directed paths $P_{1}$ and $P_{2}$ that start in $\mu(u)$ to $\alpha_{1}\left(u_{1}\right)$ and $\alpha_{1}\left(u_{2}\right)$, respectively, where $P_{1}$ contains a child $z_{1}$ of $\mu(u)$ and $P_{2}$ a child $z_{2}$ of $\mu(u)$ that is distinct from $z_{1}$. Property (A3) implies $\alpha_{1}\left(u_{1}\right) \preceq_{N} z_{1} \prec_{N} \alpha_{1}(u)$ and $\alpha_{1}\left(u_{2}\right) \preceq_{N} z_{2} \prec_{N} \alpha_{1}(u)$. The construction of $\mu$ implies that $\mu\left(u_{1}\right)$ is always located on the path $P_{2}$ or the path $P_{1} x$ for some child $x$ of $\alpha_{1}\left(u_{1}\right)$ (if $\alpha_{1}\left(u_{1}\right)$ is not a leaf) and $\mu\left(u_{2}\right)$ is always located on the path $P_{1}$ or the path $P_{2} y$ for some child $y$ of $\alpha_{1}\left(u_{2}\right)$ (if $\alpha_{1}\left(u_{2}\right)$ is not a leaf). Moreover, Property (R3) implies $\mu\left(u_{1}\right), \mu\left(u_{2}\right) \prec_{N} \mu(u)$. Taken the latter two arguments together, we obtain $\mu(u) \in Q_{N}\left(\mu\left(u_{1}\right), \mu\left(u_{2}\right)\right)$.

Thus, $\mu$ is a TreeNet-reconciliation map from $\left(T ; t^{\alpha}, \sigma\right)$ to $N$.

Note that Proposition 1 implies that the definition of a TreeNet-reconciliation map applied to binary gene trees and binary networks is a natural generalization of the concept of a biTreeNetreconciliation map.

\section{Reconciliation Maps to Trees}

In this section, we consider the special case of reconciliation maps where the network is a tree. As described in the introduction, the reconciliation of gene trees (with or without event-labels) with species trees has been the subject of numerous studies. In [26] (tree) reconciliation maps between not necessarily binary event-labeled gene trees and species trees were studied. In particular, a tree reconciliation map $\mu: V \rightarrow W \cup F$ from a given event-labeled gene tree $(T=(V, E) ; t, \sigma)$ to a species tree $S=(W, F)$ is defined there which, in our notation, is equivalent to replacing Property (R2.i) in Definition 2 by

$$
\text { (R2.i*) If } x \in V \text { and } t(x)=\mathfrak{s} \text {, then } \mu(x)=\operatorname{lca}_{S}\left(\sigma\left(L_{T}(x)\right)\right),
$$

and by adding the constraint

(R2.iii) If $x \in V^{0}$ and $t(x)=\mathfrak{s}$, then $\mu\left(y_{1}\right)$ and $\mu\left(y_{2}\right)$ are incomparable in $S$

for any two distinct children $y_{1}$ and $y_{2}$ of $x$.

If a map $\mu$ from a given event-labeled gene tree $(T ; t, \sigma)$ to a species tree $S$ satisfies Properties (R1), (R2.i. $)$, (R2.ii) and (R3) but not necessarily (R2.iii), then we shall call $\mu$ a relaxed tree reconciliation map (thus, any tree reconciliation map is also relaxed). An example of a relaxed tree reconciliation map that is not a tree reconciliation map is given in Fig. 1. In what follows, we show for the case that the species network is in fact a species tree that the concepts of a TreeNet-reconciliation map and a relaxed tree reconciliation maps are equivalent (Theorem 1).

We first present some lemmas for later use.

Lemma 1. Given any phylogenetic tree $T=(V, E)$ and any two vertices $v, w \in V$ we have either $Q_{T}(v, w)=\emptyset$ or $Q_{T}(v, w)=\left\{\operatorname{lca}_{T}(v, w)\right\}$.

In particular, $Q_{T}(v, w)=\emptyset$ if and only if $v$ and $w$ are comparable in $T$ 
Proof. Clearly, if $v=w$, then $Q_{T}(v, w)=\emptyset$, since $T$ is a tree. Thus, let $v \neq w$. Note, any two vertices $v, w \in V$ are either comparable in $T$ or not.

Let us first assume that $v$ and $w$ are comparable. W.l.o.g. let $v \succ_{T} w$. Thus, there is a unique path from $\operatorname{lca}_{T}(v, w)=v$ to $w$ in $T$. Therefore, $v$ and $w$ cannot be separated by any vertex in $V$. Hence, $Q_{T}(v, w)=\emptyset$.

If $v$ and $w$ are incomparable, then $\operatorname{lca}_{T}(v, w) \notin\{v, w\}$. Hence, there is a unique directed path from $\operatorname{lca}_{T}(v, w)$ to $v$ and a unique path from $\operatorname{lca}_{T}(v, w)$ to $w$ that have only the vertex $\operatorname{lca}_{T}(v, w)$ in common. Thus, $\operatorname{lca}_{T}(v, w) \in Q_{T}(v, w)$. Since $\operatorname{lca}_{T}(v, w)$ is the only vertex in $T$ that separates $v$ and $w$, we have $Q_{T}(v, w)=\left\{\operatorname{lca}_{T}(v, w)\right\}$.

Lemma 2. Let $(T=(V, E) ; t, \sigma)$ be an event-labeled gene tree, let $S=(W, F)$ be a species tree on $\mathbb{S}$, and let $x \in V$ with $t(x)=\mathfrak{s}$ and children $x_{1}, \ldots, x_{k}, k \geq 2$, in $T$. Suppose $\mu$ is a TreeNetreconciliation map from $(T ; t, \sigma)$ to $S$. Then, $\mu(x) \succeq_{S}$ q for all $q \in Q_{S}^{2}\left(\mu\left(x_{1}\right), \ldots, \mu\left(x_{k}\right)\right)$, i.e., $\mu(x)$ is the $\succeq_{S}$-maximal element in $Q_{S}^{2}\left(\mu\left(x_{1}\right), \ldots, \mu\left(x_{k}\right)\right)$

Proof. For simplicity, for every $x_{i} \in V$ let $v_{i}$ be either the vertex $\mu\left(x_{i}\right)$, if $\mu\left(x_{i}\right) \in W$ or the vertex $h_{S}(e)$ if $\mu\left(x_{i}\right)=e \in F, 1 \leq i \leq k$. Moreover, put $Q_{S}^{2}:=Q_{S}^{2}\left(\mu\left(x_{1}\right), \ldots, \mu\left(x_{k}\right)\right)$.

We show first that there is always a vertex $q^{*} \in Q_{S}^{2}$ with $q^{*} \succeq_{S} q$ for all $q \in Q_{S}^{2}$. Property (R2.i) implies that $Q_{S}^{2} \neq \emptyset$. If $\left|Q_{S}^{2}\right|=1$, then the lemma trivially holds. Now, assume that there are distinct $q, q^{\prime} \in Q_{S}^{2}$ that are incomparable. By Lemma $1, q=\operatorname{lca}\left(v_{i}, v_{j}\right)$ and $q^{\prime}=\operatorname{lca}_{S}\left(v_{r}, v_{s}\right)$ where $v_{i}$ and $v_{j}$ as well as $v_{r}$ and $v_{s}$ are incomparable in $S$ and $i, j, r, s \in\{1, \ldots, k\}$. Since $S$ is a tree, we have $\operatorname{lca}_{S}\left(q, q^{\prime}\right)=\operatorname{lca}\left(v_{i}, v_{r}\right)$. Thus, $\operatorname{lca}_{S}\left(q, q^{\prime}\right)$ separates $v_{i}$ and $v_{r}$ and therefore, $q^{\prime \prime}:=\operatorname{lca}_{S}\left(q, q^{\prime}\right) \in Q_{S}^{2}$. In other words, for any two vertices $q, q^{\prime} \in Q_{S}^{2}$ there is always a vertex $q^{\prime \prime} \in Q_{S}^{2}$ with $q^{\prime \prime} \succeq_{S} q, q^{\prime}$. The latter arguments imply that there is a unique $\succeq_{S}$-maximal element $q^{*}$ in $Q_{S}^{2}$ that is an ancestor of all elements in $Q_{S}^{2}$, as required.

Property (R2.i) implies that $\mu(x) \in Q_{S}^{2}$. By Lemma 1 and since $S$ is a tree, there are two distinct children $x_{r}$ and $x_{s}$ of $x$ such that $\mu(x) \in Q_{S}\left(\mu\left(x_{r}\right), \mu\left(x_{s}\right)\right)$ and $\mu(x)=\operatorname{lca}_{S}\left(v_{r}, v_{s}\right)$. It remains to show that $q^{*}=\mu(x)$. Assume for contradiction that $q^{*} \neq \mu(x)$ and thus, $q^{*} \succ_{S} \mu(x)$. Again by Lemma $1, q^{*}=\operatorname{lca}_{S}\left(v_{i}, v_{j}\right)$ for some $1 \leq i<j \leq k$. Since $q^{*}$ separates $v_{i}$ and $v_{j}$ and $q^{*} \succ_{S} \mu(x)$ at least one of $v_{i}$ and $v_{j}$, say $v_{i}$, must be incomparable with $\mu(x)$. Since $x_{i}$ is a child of $x$ in $T$ we can apply Property (R3) to conclude that $\mu(x) \succ_{S} \mu\left(x_{i}\right) \succeq_{S} v_{i}$. Hence, $v_{i}$ and $\mu(x)$ must be comparable in $S$, a contradiction.

Lemma 3. Let $(T=(V, E) ; t, \sigma)$ be an event-labeled gene tree such that there exists a relaxed tree reconciliation map $\mu$ from $(T ; t, \sigma)$ to some species tree $S$. Let $x \in V$ with $t(x)=\mathfrak{s}$. Then, there exists two distinct children $x_{i}$ and $x_{j}$ of $x$ in $T$ that satisfy the following two properties,

(i) $\mu(x)=\operatorname{lca}_{S}\left(\mu\left(x_{i}\right), \mu\left(x_{j}\right)\right)$, and

(ii) $\mu\left(x_{i}\right)$ and $\mu\left(x_{j}\right)$ are incomparable in $S$.

Furthermore, for any child $x_{i}$ of $x$ in $T$ there is a child $x_{j}$ of $x$ in $T$ such that

(iii) $\sigma\left(L_{T}\left(x_{i}\right)\right) \cap \sigma\left(L_{T}\left(x_{j}\right)\right)=\emptyset$.

Proof. Let $x \in V$ with $t(x)=\mathfrak{s}$ and children $x_{1}, \ldots, x_{k}, k \geq 2$, in $T$ and put $S=(W, F)$.

We combine the proof of Statements (i) and (ii). Property (R2. $i^{*}$ ) implies that $\mu(x)=$ lca $_{S}\left(\sigma\left(L_{T}(x)\right)\right)$. Clearly, $\mu(x)$ can be expressed as $\mu(x)=\operatorname{lca}_{S}\left(z_{1}, z_{2}\right)$ for some leaves $z_{1}, z_{2} \in$ $\sigma\left(L_{T}(x)\right)$. Since $\sigma\left(L_{T}(x)\right)=\bigcup_{i=1}^{k} \sigma\left(L_{T}\left(x_{i}\right)\right)$, there exist children of $x$ in $T$, say w.l.o.g. $x_{1}$ and $x_{2}$, with $z_{1} \in \sigma\left(L_{T}\left(x_{1}\right)\right)$ and $z_{2} \in \sigma\left(L_{T}\left(x_{2}\right)\right)$. Note that $x_{1}$ and $x_{2}$ must be distinct since $x_{1}=x_{2}$ and Property (R3) would imply that $\mu(x) \succ_{S} \mu\left(x_{1}\right) \succeq_{S} \operatorname{lca}_{S}\left(z_{1}, z_{2}\right)$ and thus, $\mu(x) \neq \operatorname{lca}_{S}\left(z_{1}, z_{2}\right)$; a contradiction. The latter together with $\mu(x)=\operatorname{lca}_{S}\left(z_{1}, z_{2}\right) ; \mu(x) \succ_{S} \mu\left(x_{1}\right) \succeq_{S} z_{1}$; and $\mu(x) \succ_{S}$ $\mu\left(x_{2}\right) \succeq_{S} z_{2}$ implies that $\mu\left(x_{1}\right)$ and $\mu\left(x_{2}\right)$ must be incomparable in $S$ and lca $S\left(\mu\left(x_{1}\right), \mu\left(x_{2}\right)\right)=$ $\mu(x)$. This establishes Statements (i) and (ii). 
It remains to show that Statement (iii) holds. Assume for contradiction that there is a child of $x$, say $x_{1}$, such that $\sigma\left(L_{T}\left(x_{1}\right)\right) \cap \sigma\left(L_{T}\left(x_{j}\right)\right) \neq \emptyset, 2 \leq j \leq k$. By Property (R3), we have $\mu(x) \succ_{S} \mu\left(x_{1}\right)$.

Let $e$ be the first arc on the (unique) directed path from $\mu(x)$ to $\mu\left(x_{1}\right)$ in $S$. Since $\mu(x) \succ_{S} \mu\left(x_{1}\right)$ and Property (R2) holds, we have $e \succeq_{S} \mu\left(x_{1}\right)$. By Property (R3), we have $e \succeq_{S} \mu\left(x_{1}\right) \succeq_{S} z$ for all $z \in \sigma\left(L_{T}\left(x_{1}\right)\right)$, and therefore, $y:=h_{S}(e) \succeq_{S} z$ for all $z \in \sigma\left(L_{T}\left(x_{1}\right)\right)$. In other words, any path from $\mu(x)$ to $z$ must contain the arc $e$ for all $z \in \sigma\left(L_{T}\left(x_{1}\right)\right)$

By assumption, there is a leaf $z \in \sigma\left(L_{T}\left(x_{1}\right)\right) \cap \sigma\left(L_{T}\left(x_{2}\right)\right)$. By Property (R3) and the latter arguments, $\mu(x) \succ_{S} e \succeq_{S} \mu\left(x_{2}\right) \succeq_{S} z$ Again, Property (R3) implies that $\mu\left(x_{2}\right) \succeq_{S} z^{\prime}$ for all $z^{\prime} \in$ $\sigma\left(L_{T}\left(x_{2}\right)\right)$, and therefore, $y \succeq_{S} z^{\prime}$ for all $z^{\prime} \in \sigma\left(L_{T}\left(x_{2}\right)\right)$. Hence, any path from $\mu(x)$ to $z^{\prime}$ must contain the arc $e$ for all $z^{\prime} \in \sigma\left(L_{T}\left(x_{2}\right)\right)$. Repeating the latter arguments shows that any path from $\mu(x)$ to $z^{\prime}$ must contain the arc $e$ for all $z^{\prime} \in \sigma\left(L_{T}\left(x_{i}\right)\right)$ and $i \in\{1, \ldots, k\}$. Thus, $y \succeq_{S} z^{\prime}$ for all $z^{\prime} \in \bigcup_{i=1}^{k} \sigma\left(L_{T}\left(x_{i}\right)\right)=\sigma\left(L_{T}(x)\right)$. Hence, $\mu(x) \succ_{S} y \succeq_{S} \operatorname{lca}_{S}\left(\sigma\left(L_{T}(x)\right)\right)$; a contradiction to Property $\left(\mathrm{R} 2 \cdot \mathrm{i}^{*}\right)$.

We are now in the position to prove the aforementioned equivalence between TreeNetreconciliation and relaxed tree reconciliation maps.

Theorem 1. Let $(T=(V, E) ; t, \sigma)$ be an event-labeled gene tree, $S=(W, F)$ be a species tree, and $\mu: V \rightarrow W \cup F$ be a map. Then, $\mu$ is a relaxed tree reconciliation map from $(T ; t, \sigma)$ to $S$ if and only if $\mu$ is a TreeNet-reconciliation map from $(T ; t, \sigma)$ to $S$.

Proof. Note first that all Properties (R1), (R2.ii) and (R3) in Definition 2 remain the same for both, TreeNet-reconciliation maps and relaxed tree reconciliation maps. Hence, it suffices to show that if $\mu$ is a TreeNet-reconciliation map then Property ( $\left.2 . i^{*}\right)$ holds and that if $\mu$ is a relaxed tree reconciliation then Property (R2.i) holds.

Assume first that $\mu$ is a relaxed tree reconciliation map from $(T ; t, \sigma)$ to $S$. Let $x \in V$ be a vertex with $t(x)=\mathfrak{s}$ and children $x_{1}, \ldots, x_{k}, k \geq 2$, in $T$. Then Lemma 3(i) implies that $\mu(x)=$ $\operatorname{lca}_{S}\left(\mu\left(x_{i}\right), \mu\left(x_{j}\right)\right)$, for some $i, j \in\{1, \ldots, k\}$. By Lemma $1, Q_{S}\left(\mu\left(x_{i}\right), \mu\left(x_{j}\right)\right)=\{\mu(x)\}$ follows. Hence, $\mu(x) \in Q_{S}^{2}\left(\mu\left(x_{1}\right), \ldots, \mu\left(x_{k}\right)\right)$. Thus, Property (R2.i) holds.

Now assume that $\mu$ is TreeNet-reconciliation map from $(T ; t, \sigma)$ to $S$. In what follows we will make use of the following observation: If $y_{1}$ and $y_{2}$ are incomparable in $S$ and $y=\operatorname{lca}_{S}\left(y_{1}, y_{2}\right)$ and $u, v, w \in W$ are such that $y \succeq_{S} u, y_{1} \succeq_{S} v$ and $y_{2} \succeq_{S} w$, then

$$
y=\operatorname{lca}_{S}(v, w)=\operatorname{lca}_{S}(u, v, w)=\operatorname{lca}_{S}\left(y_{1}, y_{2}, w\right) .
$$

Property (R2.i) and Lemma 1 imply that $\mu(x)=\operatorname{lca}_{S}\left(\mu\left(x_{i}\right), \mu\left(x_{j}\right)\right) \in Q_{S}^{2}\left(\mu\left(x_{1}\right), \ldots, \mu\left(x_{k}\right)\right)$ for some distinct $i, j \in\{1, \ldots, k\}$. W.l.o.g., let $\mu(x)=\operatorname{lca}_{S}\left(\mu\left(x_{1}\right), \mu\left(x_{2}\right)\right) \in Q_{S}^{2}\left(\mu\left(x_{1}\right), \ldots, \mu\left(x_{k}\right)\right)$. Property (R3) and Lemma 2 imply that $\mu(x) \succeq_{S} \operatorname{lca}_{S}\left(\mu\left(x_{r}\right), \mu\left(x_{s}\right)\right)$ for all $r, s \in\{1, \ldots, k\}$. Repeated application of Equ. (1) implies that

$$
\mu(x)=\operatorname{lca}_{S}\left(\mu\left(x_{1}\right), \mu\left(x_{2}\right), \operatorname{lca}_{S}\left(\mu\left(x_{r}\right), \mu\left(x_{s}\right)\right)\right)=\operatorname{lca}_{S}\left(\mu\left(x_{1}\right), \mu\left(x_{2}\right), \mu\left(x_{r}\right), \mu\left(x_{s}\right)\right)
$$

for all $r, s \in\{1, \ldots, k\}$ and, again by repeated application of Equ. (1), we obtain

$$
\mu(x)=\operatorname{lca}_{S}\left(\mu\left(x_{1}\right), \ldots, \mu\left(x_{k}\right)\right) .
$$

Now, put $v_{i}:=\operatorname{lca}_{S}\left(\sigma\left(L_{T}\left(x_{i}\right)\right)\right), 1 \leq i \leq k$. Property (R3) implies that $\mu\left(x_{r}\right) \succeq_{S} z$ for all $z \in \sigma\left(L_{T}\left(x_{r}\right)\right)$ and $1 \leq r \leq k$. Thus, $\mu\left(x_{1}\right) \succeq_{S} v_{1}$ and $\mu\left(x_{2}\right) \succeq_{S} v_{2}$. Since $\mu\left(x_{1}\right)$ and $\mu\left(x_{2}\right)$ are separated by $\mu(x)$, they must be incomparable. Equ. (1) implies that

$$
\mu(x)=\operatorname{lca}_{S}\left(v_{1}, v_{2}\right) .
$$

Again, by Property (R3), we have $\mu(x) \succ_{S} \mu\left(x_{r}\right)$ and $\mu\left(x_{r}\right) \succeq_{S} \operatorname{lca}_{S}\left(\sigma\left(L_{T}\left(x_{r}\right)\right)\right), 1 \leq r \leq k$. Hence, Equ. (1) implies that

$$
\mu(x)=\operatorname{lca}_{S}\left(v_{1}, v_{2}, v_{r}\right) \text { for all } r \in\{1, \ldots, k\} .
$$


Repeated application of Equ. (1) implies

$$
\mu(x)=\operatorname{lca}_{S}\left(v_{1}, v_{2}, v_{3}, v_{4}, \ldots, v_{k}\right) .
$$

To make the final step, we first observe that for non-empty vertex sets $A, B$ of a tree, lca $(A \cup B)=$ lca $(\mathrm{lca}(A), \operatorname{lca}(B))$ holds. The latter and $\sigma\left(L_{T}(x)\right)=\bigcup_{i=1}^{k} \sigma\left(L_{T}\left(x_{i}\right)\right)$ allows us to rewrite Equ. (2) as $\mu(x)=\operatorname{lca}_{S}\left(\sigma\left(L_{T}(x)\right)\right)$. Therefore, the map $\mu$ satisfies Property (R2..i*).

As an immediate consequence of Theorem 1 we obtain

Corollary 1. Suppose $(T(V, E) ; t, \sigma)$ is an event-labeled gene tree, $S=(W, F)$ is a species tree and $\mu: V \rightarrow W \cup F$ is a map. Then $\mu$ is a tree reconciliation map from $(T ; t, \sigma)$ to $a S$ if and only if $\mu$ is a TreeNet-reconciliation map from $(T ; t, \sigma)$ to $S$ that additionally satisfies Property (R2.iii).

As a consequence of Lemma 3(ii), if $\mu$ is a relaxed tree reconciliation map between a binary gene tree and a (not necessarily binary) species tree, then Property (R2.iii) is always satisfied. Thus any relaxed tree reconciliation map between a binary gene tree and a species tree is also a tree reconciliation map. Therefore, Theorem 1 implies

Corollary 2. Suppose $(T ; t, \sigma)$ is a binary event-labeled gene tree, $S=(W, F)$ is a species tree and $\mu: V \rightarrow W \cup F$ is a map. Then, $\mu$ is a tree reconciliation map from $(T ; t, \sigma)$ to $S$ if and only if $\mu$ is a TreeNet-reconciliation map from $(T ; t, \sigma)$ to $S$.

\section{Informative Triples}

It is of interest to understand when a species tree or species network exists for a given gene tree $(T ; t, \sigma)$, and if it does exist, which constraints (if any) such an network imposes on $(T ; t, \sigma)$ for different kinds of networks. In this section, we shall consider constraints that are given in terms of triples. We start with briefly recalling the terminology surrounding them.

A triple $a b \mid c$ is a binary reduced tree $T$ on three leaves $a, b$ and $c$ such that the path from $a$ to $b$ does not intersect the path from $c$ to the root $\rho_{T}$. A network $N$ on $X$ displays a triple $a b \mid c$, if $a, b, c \in X$ and $a b \mid c$ can be obtained from $N$ by deleting arcs and vertices, and suppressing vertices with in- and outdegree one. Note, that no distinction is made between $a b \mid c$ and $b a \mid c$. We write $R(N)$ for the set of all triples that are displayed by the network $N$. A set $R$ of triples is compatible if there is a phylogenetic tree $T$ on $X=\bigcup_{t \in R} L(t)$ such that $R \subseteq R(T)$.

Interestingly, the existence of tree reconciliation maps from event-labeled gene trees $(T ; t, \sigma)$ to some species tree can be characterized in terms of underlying "informative species triples" displayed by the gene tree, $[26,28]$, that is, in terms of the set

$$
\begin{array}{r}
\mathcal{S}(T ; t, \sigma):=\left\{\sigma(a) \sigma(b)|\sigma(c): a b| c \in R(T), t\left(\operatorname{lca}_{T}(a, b, c)\right)=\mathfrak{s},\right. \text { and } \\
\sigma(a), \sigma(b), \sigma(c) \text { are pairwise distinct }\}
\end{array}
$$

In particular, in [28, Theorem 8] the existence of relaxed tree reconciliation maps for binary event-labeled gene trees $(T ; t, \sigma)$ is characterized in terms of the compatibility of $\mathcal{S}(T ; t, \sigma)$ under the following assumption: For any vertex $x$ of $T$ with $t(x)=\mathfrak{s}$ and children $x_{1}$ and $x_{2}$ we have $\sigma\left(L_{T}\left(x_{1}\right)\right) \cap \sigma\left(L_{T}\left(x_{2}\right)\right)=\emptyset$. Lemma 3(iii) implies that this condition is always satisfied for relaxed tree reconciliation maps and, therefore, also by tree reconciliation maps between such trees. The latter argument combined with Corollary 2 and Theorem 1 therefore immediately implies the following mild generalization of the aforementioned characterization from [28]:

Theorem 2. Let $(T=(V, E) ; t, \sigma)$ be an event-labeled binary gene tree and let $S$ be a species tree. Then, there exists a relaxed tree reconciliation map $\mu$ from $(T ; t, \sigma)$ to $S$ (or equivalently a TreeNet-reconciliation map $\mu$ from $(T ; t, \sigma)$ to $S)$ if and only if $\mathcal{S}(T ; t, \sigma)$ is compatible and, for any $x \in V$ with $t(x)=\mathfrak{s}$ with children $x_{1}$ and $x_{2}$, we have $\sigma\left(L_{T}\left(x_{1}\right)\right) \cap \sigma\left(L_{T}\left(x_{2}\right)\right)=\emptyset$. 

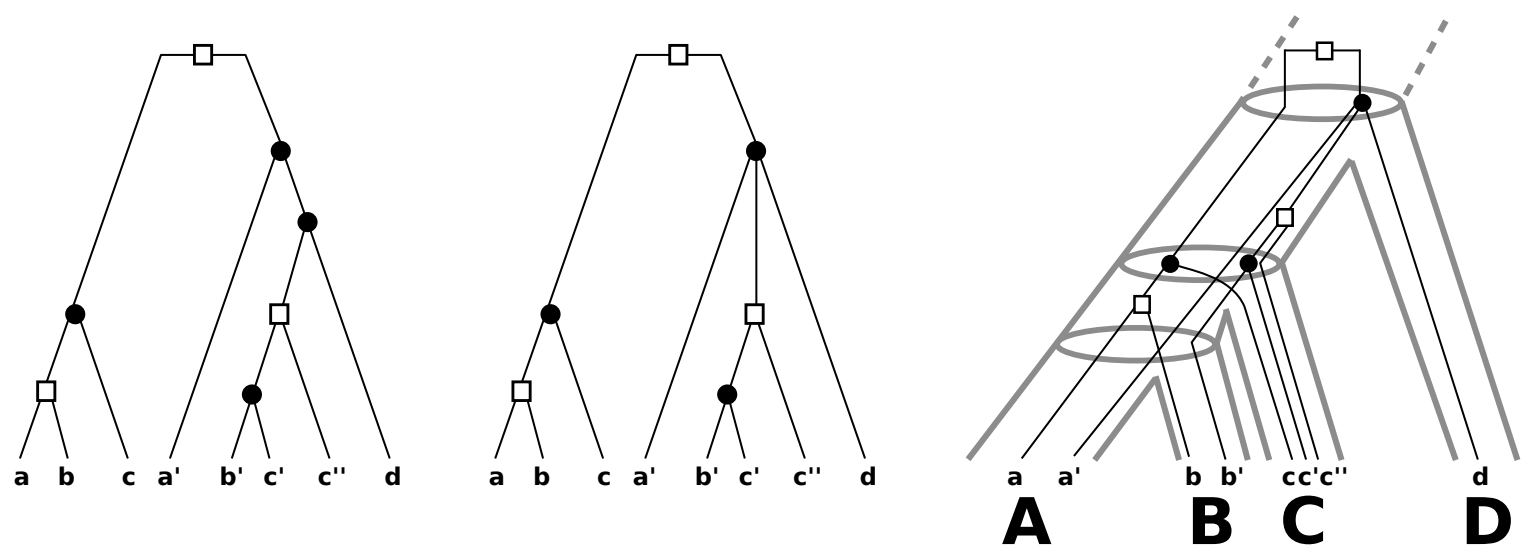

Figure 1: (Adapted from [26, Fig. 3]) Two event-labeled gene trees $\left(T^{\prime} ; t^{\prime}, \sigma\right)$ (left) and $(T ; t, \sigma)$ (middle) on a set $\mathbb{G}=\left\{a, a^{\prime}, b, b^{\prime}, c, c^{\prime}, c^{\prime \prime}, d\right\}$. Speciation and duplication events are represented as $\bullet$ and $\square$, respectively. The relaxed tree reconciliation map from $(T ; t, \sigma)$ to the species tree $S$ on $\mathbb{S}=\{A, B, C, D\}$ is implicitly shown by drawing the gene tree inside the tube-like species tree $S$ (right). Here, $\sigma$ maps each gene in $\mathbb{G}$ to the species (capitals below the genes) $A, B, C, D \in \mathbb{S}$. Although there is a relaxed tree reconciliation map from the non-binary gene tree $(T ; t, \sigma)$ to $S$, there exists no tree reconciliation map from $(T ; t, \sigma)$ to any species tree, since $A B|C, B C| A \in \mathcal{S}(T ; t, \sigma)$ implies that $\mathcal{S}(T ; t, \sigma)$ is incompatible (cf. Thm. 3). Moreover, there exists neither a (relaxed) tree reconciliation map nor a TreeNetreconciliation map from the binary gene tree $\left(T^{\prime} ; t^{\prime}, \sigma\right)$ to any species tree, since the set $\mathcal{S}\left(T^{\prime} ; t^{\prime}, \sigma\right)$ contains the triples $A B \mid C$ and $B C \mid A$ and is, therefore, incompatible (cf. Thm. 2).

It is worth mentioning that a relaxed tree reconciliation map $\mu$ exists for a gene tree $(T ; t, \sigma)$ that satisfies the conditions in Theorem 2 to any species tree $S$ that displays all triples in $\mathcal{S}(T ; t, \sigma)$ [28]. Moreover, this map can be computed in polynomial time [28].

Note that the main result in [28, Theorem 8] was generalized in [26] for non-binary eventlabeled gene trees as follows.

Theorem 3 ([26, Thm. 5.7]). Let $(T=(V, E) ; t, \sigma)$ be an event-labeled gene tree such that for every vertex $x$ in $T$ with $t(x)=\mathfrak{s}$ and children $x_{1} \ldots, x_{k}, k \geq 2$, we have $\sigma\left(L_{T}\left(x_{i}\right)\right) \cap \sigma\left(L_{T}\left(x_{j}\right)\right)=\emptyset$, $1 \leq i<j \leq k$. Then, there exists a tree reconciliation map $\mu$ from $(T ; t, \sigma)$ to some species tree $S$ if and only if $\mathcal{S}(T ; t, \sigma)$ is compatible.

One might hope to obtain similar characterizations for relaxed tree reconciliation maps or TreeNet-reconciliation maps for non-binary event-labeled gene trees. However, this does not appear to be straight-forward.

In particular, in Fig. 1 an example is presented (based on [26, Fig. 3]) which shows that the compatibility of $\mathcal{S}(T ; t, \sigma)$ is not necessary for the existence of a relaxed tree reconciliation map for a non-binary event-labeled gene tree $(T ; t, \sigma)$, even though the conditions in Theorem 3 for $(T ; t, \sigma)$ are met. Thus, one might be inclined to try and obtain at least a characterization of TreeNet-reconciliation maps in terms of "informative" species triples from a binary gene tree. However, this does not seem to be as straight-forward as it might sound in view of the examples given in Figs. 2 and 3.

More precisely, consider the event-labeled binary gene tree $(T ; t, \sigma)$ in Fig. 2 (left). For this tree we have $\sigma(a)=\sigma\left(a^{\prime}\right)=\sigma\left(a^{\prime \prime}\right)=A, \sigma(b)=\sigma\left(b^{\prime}\right)=\sigma\left(b^{\prime \prime}\right)=B, \sigma(c)=C$ and $\sigma(d)=$ $\sigma\left(d^{\prime}\right)=D$. Therefore, $A B|D, B D| A \in \mathcal{S}(T ; t, \sigma)$ and hence, the set $\mathcal{S}(T ; t, \sigma)$ is incompatible. Thus, no (relaxed) tree reconciliation or TreeNet-reconciliation map from $(T ; t, \sigma)$ to a species tree can exist in view of Theorem 2. Fig. 2 (right) shows a network $N$ on $\mathbb{S}=\{A, B, C, D\}$. There is a TreeNet-reconciliation map from $(T ; t, \sigma)$ to $N$, which is implicitly shown in Fig. 2 (right) by drawing $(T ; t, \sigma)$ inside $N$. Here, $N$ does not display the species triple $A B \mid C \in \mathcal{S}(T ; t, \sigma)$. Thus, it is not necessary that each triple of $\mathcal{S}(T ; t, \sigma)$ is displayed by $N$ for the existence of a TreeNet- 

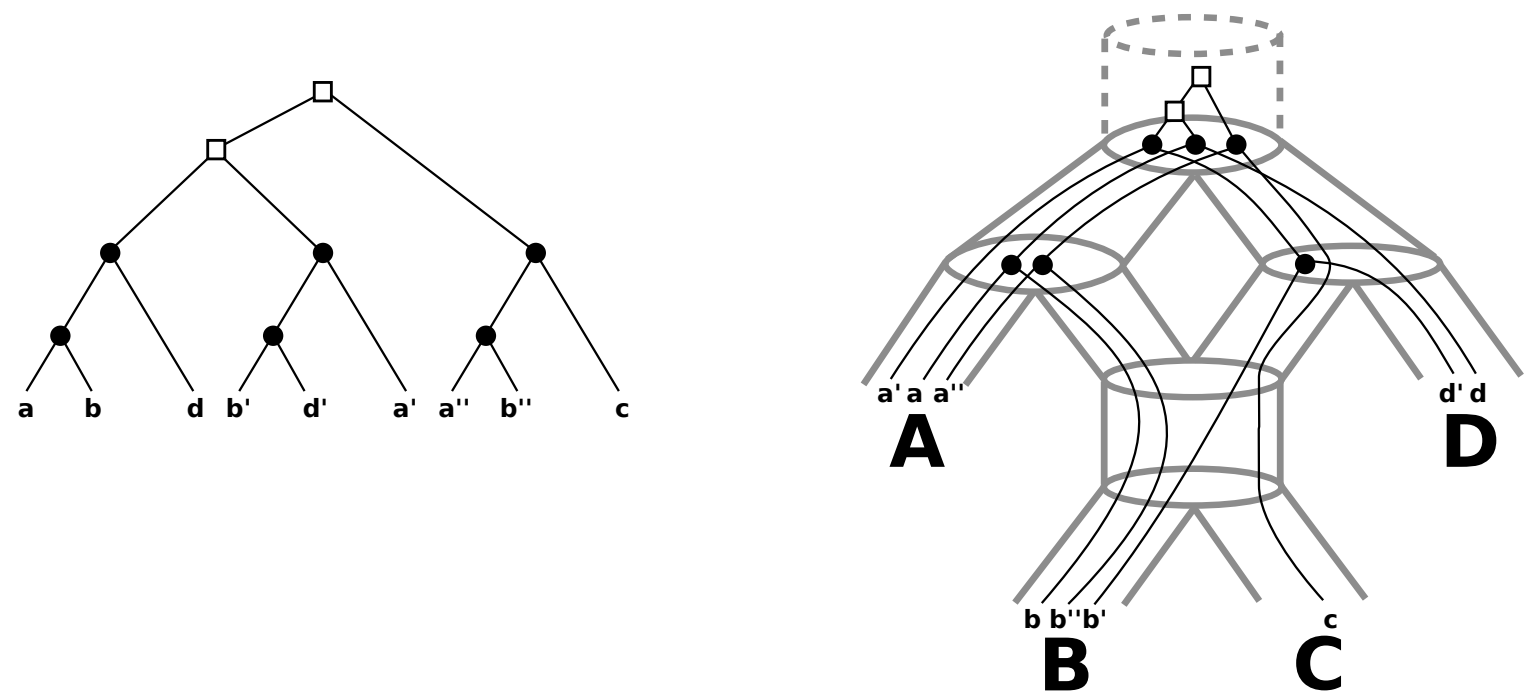

Figure 2: Left, an event-labeled binary gene tree $(T ; t, \sigma)$ on a set $\mathbb{G}=\left\{a, a^{\prime}, a^{\prime \prime}, b, b^{\prime}, b^{\prime \prime}, c, d, d^{\prime}\right\}$ of genes is shown. Speciation and duplication events are represented as $\bullet$ and $\square$, respectively. For $(T ; t, \sigma)$ there is neither a (relaxed) tree reconciliation nor a TreeNet-reconciliation map to any species tree. Right, a tube-like network $N$ on $\mathbb{S}=\{A, B, C, D\}$ is shown. There is a TreeNet-reconciliation map from $(T ; t, \sigma)$ to $N$, which is implicitly shown by drawing the gene tree inside $N$. See text for further details.
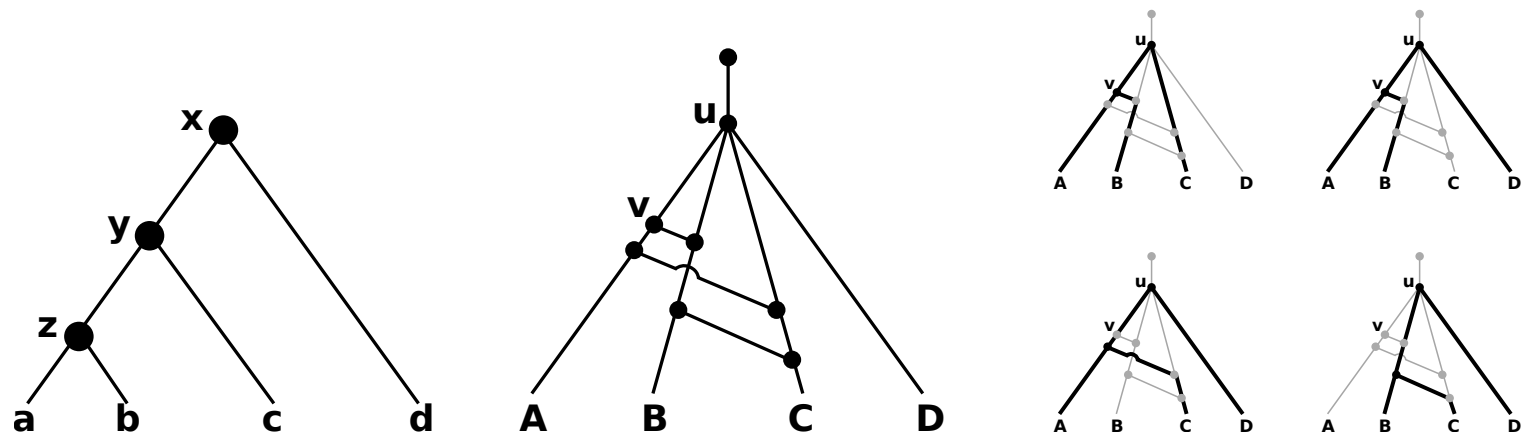

Figure 3: Left, an event-labeled binary gene tree $(T ; t, \sigma)$ on a set $\mathbb{G}=\{a, b, c, d\}$ of genes where all inner vertices are speciation vertices. The species network (middle) displays all triples in $\mathcal{S}(T ; t, \sigma)=$ $\{A B|C, A B| D, A C|D, B C| D\}$ as highlighted in the right part. However, there is no reconciliation map $\mu$ from $(T ; t, \sigma)$ to $N$. See text for further details. 
reconciliation map.

Now consider the event-labeled binary gene tree $(T ; t, \sigma)$ as in Fig. 3 (left). Here, $\sigma(a)=A$, $\sigma(b)=B, \sigma(c)=C$, and $\sigma(d)=D$. The network $N=(W, F)$ in Fig. 3 (middle) displays all triples in $\mathcal{S}(T ; t, \sigma)=\{A B|C, A B| D, A C|D, B C| D\}$. However, there is no TreeNet-reconciliation map $\mu$ from $(T ; t, \sigma)$ to $N$. To see this, assume for contradiction that there is a TreeNet-reconciliation map $\mu$. Observe first that each gene is mapped to the corresponding species (cf. Property (R1)). Due to Property (R3), we have $\mu(x) \succ_{N} \mu(d)=D$. The latter together with Property (R2.i) implies that only $\mu(x)=u$ is possible. In addition Property (R3) implies that $\mu(x) \succ_{N} \mu(y) \succ_{N} A, B, C$ and hence, only $\mu(y)=v$ is possible. However, Properties (R2.i) and (R3) imply that $\mu(z) \in W^{0}$ and $\mu(y) \succ_{N} \mu(z) \succ_{N} A, B$, which is not possible. Thus, $N$ is not a network for $(T ; t, \sigma)$. Note, $(T ; t, \sigma)$ satisfies the assumptions in Theorem 2 and $\mathcal{S}(T ; t, \sigma)$ is compatible. Thus, Theorem 2 implies that for any species tree $S$ that displays all triples in $\mathcal{S}(T ; t, \sigma)$ there is a TreeNet-reconciliation map from $(T ; t, \sigma)$ to $S$.

\section{Unfoldings and Foldings}

In the next section we shall show that we can always reconcile a given event-labeled gene tree with some species network. To show this we shall use the concept of foldings of MUL-trees which we shall consider in this section.

We begin by recalling an unfolding operation $U$ of networks that was first proposed in [29]. For any network $N$ on $\mathbb{S}$, this operation constructs a pseudo MUL-tree $\left(U^{*}(N), \chi^{*}\right)$ as follows:

- The vertex set $V^{*}$ of $U^{*}(N)$ is the set of all directed paths $P$ in $N$ that start at the root of $N$ and end in a vertex of $N$,

- there is an arc from a vertex $P$ in $U^{*}(N)$ to a vertex $P^{\prime}$ in $U^{*}(N)$ if and only if $P^{\prime}=P a$ holds for some $\operatorname{arc} a$ in $N$, and

- and $\chi^{*}: \mathbb{S} \rightarrow 2^{L\left(U^{*}(N)\right)}-\{\emptyset\}$ is the map that assigns to all $x \in \mathbb{S}$ the set of directed paths in $V^{*}$ that end in $x$.

The MUL-tree obtained by suppressing all indegree one and outdegree one vertices in $U^{*}(N)$, if there are any, is denoted by $U(N)$. In [29], it is shown that $\left(U(N), \chi^{*}\right)$ is indeed a MUL-tree and that it is unique.

We now want to perform the converse operation and "fold up" a MUL-tree into a network. We formalize this by adapting the concept of a folding map as defined in [30, p.1771].

Definition 4 (Folding map of a MUL-tree onto a network). Given a pseudo MUL-tree $(M=$ $(D, U), \chi)$ labeled by $\mathbb{S}$, and a network $N=(W, F)$ on $\mathbb{S}$, a folding map $f:(M, \chi) \rightarrow N$ is a pair of surjective functions $f_{\mathcal{V}}: D \rightarrow W$ and $f_{\mathcal{E}}: U \rightarrow F$ such that

(F1) for all $a \in U$ we have $t_{N}\left(f_{\mathcal{E}}(a)\right)=f_{\mathcal{V}}\left(t_{M}(a)\right)$ and $h_{N}\left(f_{\mathcal{E}}(a)\right)=f_{\mathcal{V}}\left(h_{M}(a)\right)$,

(F2) if $v \in L(M)$ with $v \in \chi(x)$ for some $x \in \mathbb{S}$ then $f_{\mathcal{V}}(v)=x$, and

(F3) for each arc $a \in F$ and $v \in D$ such that $f_{\mathcal{V}}(v)=t_{N}(a)$ there is a unique arc $\widetilde{a}^{v} \in U$ (the lifting of the arc $a$ at $v)$ such that $f_{\mathcal{E}}\left(\widetilde{a^{v}}\right)=a$ and $t_{M}\left(\widetilde{a^{v}}\right)=v$.

We say that a MUL-tree $M$ can be folded into a network $N$, whenever there is a subdivision $M^{\prime}$ of $M$ such that there is a folding map $f$ from $M^{\prime}$ to $N$.

Informally speaking, Property (F1) ensures that the maps $f_{\mathcal{V}}$ and $f_{\mathcal{E}}$ are linked, that is, each $\operatorname{arc} a$ in $M$ is mapped to an arc $a^{\prime}$ in $N$ such that the tail and head of $a$ is mapped to the tail and head of $a^{\prime}$, respectively. Property (F2) ensures that each leaf (gene) is mapped to the species in which it resides. Finally, Property (F3) ensures that two distinct arcs of $M$ with the same tail are never mapped to the same arc in $N$. 
The following two results are restated from [30, Theorem 2 and Corollary 2], and provide a useful connection between foldings of MUL-trees into a multi-arc free network $N$ and $\left(U^{*}(N), \chi^{*}\right)$ :

Theorem 4. Let $N$ be a multi-arc free network. Then the mapping $f^{*}:\left(U^{*}(N), \chi^{*}\right) \rightarrow N$ which takes each vertex in $U^{*}(N)$ to its last vertex and each $\operatorname{arc}$ in $U^{*}(N)$ to its corresponding arc in $N$ is a folding map.

Proposition 2. Suppose that $(M, \chi)$ is a pseudo MUL-tree and $N$ is a multi-arc free network, both on $\mathbb{S}$, and that $f:(M, \chi) \rightarrow N$ is a folding map. Then $(M, \chi)$ is isomorphic with $\left(U^{*}(N), \chi^{*}\right)$.

We conclude this section with two useful results concerning folding maps. The first generalizes a result from $[29$, p. 618]. As part of this, we first present a construction that associates a network $F(M)$ to a MUL-tree $(M, \chi)$.

Definition 5. Let $(M, \chi)$ with $M=(D, U)$ be a MUL-tree on $\mathbb{S}$ and $\left(M^{\prime}, \chi\right)$ with $M^{\prime}=\left(D^{\prime}, U^{\prime}\right)$ be its simple subdivision. Now we construct a digraph $N=(W, F)$ as follows:

For each $x \in \mathbb{S}$ find all the arcs in $M^{\prime}$ whose head is contained in $\chi(x)$ and identify them to obtain a digraph $N=(W, F)$. To be more precise, for $x \in \mathbb{S}$ define $U_{x} \subseteq U$ as the set of all arcs in $M$ that are incident with a leaf $l$ with $l \in \chi(x)$. If $|\chi(x)| \geq 2$, then every arc $e=(u, v) \in U_{x}$ of $M$ is replaced by the path $\left(u, v_{e}, v\right)$ to obtain $M^{\prime}$, where $v_{e} \notin D$. For each $x \in \mathbb{S}$, let $U_{x}^{*}$ be the set of all such new arcs $\left(v_{e}, v\right)$, where $(u, v) \in U_{x}$. Hence, $\left|U_{x}^{*}\right| \geq 2$. Now, we obtain the digraph $N=(W, F)$ (with leaf set $\mathbb{S}$ and possibly with multi-arcs) by identifying for each $x \in \mathbb{S}$ all the arcs in $U_{x}^{*}$ that yield the unique arc $(\operatorname{par}(x), x)$ in $N$. By construction, all vertices in $D \backslash L(M)$ are still contained in $N$.

See Fig. 4 and 5 for illustrative examples of Definition 5. In fact, the digraph $N$ as in Definition 5 is a network as shown in the following

Lemma 4. Let $(M, \chi)$ be a MUL-tree on $\mathbb{S}$, let $\left(M^{\prime}, \chi\right)$ denote its simple subdivision and let $N$ be the digraph as in Definition 5. Then, $N$ is a network.

Proof. Let $M=(D, U), M^{\prime}=\left(D^{\prime}, U^{\prime}\right)$ and $N=(W, F)$ be the digraph as in Definition 5.

To see that $N$ is a network, note first that, by construction, $N$ satisfies Property (N2). To see Properties (N1) and (N3), suppose that $x \in W$. If $x \in D \backslash L(M)$, then, again by construction, the indegree and outdegree of $x$ in $N$ is the same as the indegree and outdegree of $x$ in $M$. Hence, Property (N1) holds in case $x$ is the root of $M$ and Property (N3) holds if $x$ is an inner vertex of $M$ distinct from the root. Now, assume that $x$ is a leaf of $N$. By construction, there is a unique arc $(y, x)$ in $N$. If $y \in D$, then, again by construction, the indegree and outdegree of $x$ in $N$ is the same as the indegree and outdegree of $x$ in $M$. So assume that $y \notin D$. Then $\left|U_{x}^{*}\right| \geq 2$. It follows that the indegree of $y$ in $N$ is at least 2. Since, again by construction, the outdegree of $y$ must be 1 , it follows that $y$ is a hybrid vertex of $N$. Thus, $N$ satisfies Property (N3). Therefore, $N$ is indeed a network.

Lemma 5. Every $M U L$-tree $(M, \chi)$ on $\mathbb{S}$ can be folded into a network on $\mathbb{S}$. The network as well as the underlying folding map can be constructed in polynomial time.

Proof. Let $(M, \chi)$ with $M=(D, U)$ be a MUL-tree on $\mathbb{S}$ and let $\left(M^{\prime}, \chi\right)$ with $M^{\prime}=\left(D^{\prime}, U^{\prime}\right)$ be its simple subdivision and let $N=(W, F)$ be the digraph as in Definition 5. By Lemma $4, N$ is a network.

We next show that there exists a folding map $f:\left(M^{\prime}, \chi\right) \rightarrow N$ of $\left(M^{\prime}, \chi\right)$ into $N$. To see this consider first the map $f_{\mathcal{V}}: D^{\prime} \rightarrow W$ given by

$$
f_{\mathcal{V}}(v)= \begin{cases}v & \text { if } v \in D \\ t_{N}\left(e^{x}\right) & \text { else. }\end{cases}
$$



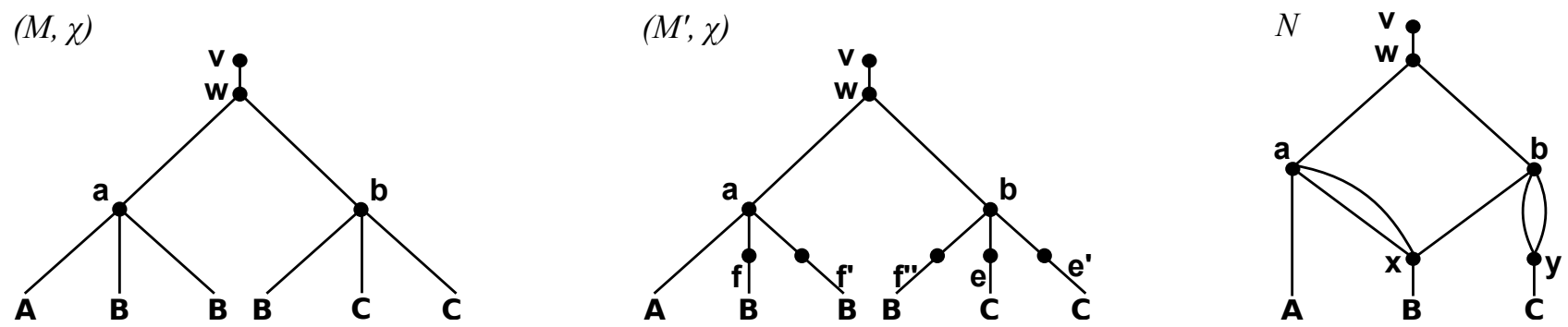

Figure 4: Left, a MUL-tree $(M, \chi)$ and its simple subdivision $\left(M^{\prime}, \chi\right)$ (middle panel). In the right panel, a network $N$ that is obtained from $\left(M^{\prime}, \chi\right)$ by identifying the two $\operatorname{arcs} e$ and $e^{\prime}$ to obtain the arc $(y, C)$ in $N$, and the $\operatorname{arcs} f, f^{\prime}$ and $f^{\prime \prime}$ to obtain the $\operatorname{arc}(x, B)$ in $N$. In addition, the identification of $f$ and $f^{\prime}$ (resp. $e$ and $e^{\prime}$ ) yields the two multi-arcs between $a$ and $x$ (resp. $b$ and $y$ ) in $N$, see Definition 5 for details. Note that $N$ is the "fold up" of $\left(M^{\prime}, \chi\right)$ and, therefore, also of $(M, \chi)$.

By construction, $f_{\mathcal{V}}$ is surjective. To obtain the map $f_{\mathcal{E}}: U^{\prime} \rightarrow F$, let us first examine in more detail the MUL-tree $M$, its simple subdivision $M^{\prime}$ and the network $N$. Let $x \in \mathbb{S}$. If $|\chi(x)| \geq 2$, then each $\operatorname{arc} e=(a, b) \in U_{x}$ in $M$ is replaced by the path $\left(a, b_{e}, b\right)$ to obtain $M^{\prime}$ and $f_{\mathcal{V}}\left(b_{e}\right)=t_{N}\left(e^{x}\right)$ and $f_{\mathcal{V}}(b)=x$. Note that $N$ may contain multi-arcs. More precisely, the construction implies that there are multi-arcs $f_{1}, \ldots, f_{\ell}, \ell \geq 2$ between two vertices $u$ and $v$ in $N$ with $v \prec_{N} u$ if and only if $f_{\mathcal{V}}^{-1}(u)=a \in D$ and $a$ is adjacent in $M$ to exactly $\ell$ leaves $b_{1}, \ldots b_{\ell}$ with $b_{i} \in \chi(x)$ for some $x \in \mathbb{S}$ and $1 \leq i \leq \ell$. In other words, there are exactly $\ell \geq 2 \operatorname{arcs} e_{1}, \ldots, e_{\ell}$ in $U_{x}$ with $t_{M}\left(e_{i}\right)=a$. Now, we put $f_{\mathcal{E}}\left(e_{i}\right)=f_{i}$ for all such $\operatorname{arcs} e_{i} \in U_{x}, 1 \leq i \leq \ell$ and $x \in \mathbb{S}$. For all other $\operatorname{arcs} e=(u, v) \in U^{\prime}$ that do not result in multi-arcs in $N$, we put $f_{\mathcal{E}}(e)=\left(f_{\mathcal{V}}(u), f_{\mathcal{V}}(v)\right)$. By construction $f_{\mathcal{E}}$ is clearly surjective. Leaving the details of the proof to the interested reader, it is also not difficult to see that $f$ satisfies Properties (F1) - (F3). Thus, $f$ is a folding map of $\left(M^{\prime}, \chi\right)$ into $N$. By Definition 4 and since $M^{\prime}$ is a subdivision of $M$, it follows that the MUL-tree $(M, \chi)$ can be folded into $N$.

Finally, all construction steps to obtain $N$ and $f$ can obviously be carried out in polynomial time.

Our second result shows that folding maps are ancestor preserving.

Lemma 6. Let $f=\left(f_{\mathcal{V}}, f_{\mathcal{E}}\right)$ be folding map from a pseudo MUL-tree $(M=(D, U), \chi)$ on $\mathbb{S}$ into a network $N=(W, F)$ on $\mathbb{S}$. Then, for any $a, b \in D \cup U$ with $a \preceq_{M} b$, we have $g(a) \preceq_{N} g(b)$ where $g(x)$ with $x \in\{a, b\}$ is $f_{\mathcal{V}}(x)$ if $x \in D$ and $f_{\mathcal{E}}(x)$ otherwise. In particular, if $a$ and $b$ are distinct and not both contained in $U$, then $g(a) \prec_{N} g(b)$.

Proof. First assume that $a, b \in U$ and $a \preceq_{M} b$. W.1.o.g. we may assume that $a \prec_{M} b$ as for $a=b$ the lemma trivially holds. Then, there is a (possibly single-vertex) directed path $P$ from $h_{N}(b)$ to $t_{N}(a)$. Let us denote the vertices crossed by $P$ by $h_{N}(b)=v_{1}, \ldots, v_{k}=t_{N}(a)$ (in order of their appearance in $P$ ) and, in case $k \geq 2$, let $e_{i}=\left(v_{i}, v_{i+1}\right) \in U, 1 \leq i<k$. Moreover, let $v_{0}, v_{k+1} \in D$ such that $b=\left(v_{0}, v_{1}\right)$ and $a=\left(v_{k}, v_{k+1}\right)$. By Property (F1), we have $f_{\mathcal{V}}\left(v_{i+1}\right) \prec_{N} f_{\mathcal{V}}\left(v_{i}\right)$ for all $0 \leq i \leq k$. Therefore, $f_{\mathcal{E}}(a) \prec_{M} f_{\mathcal{E}}\left(e_{k-1}\right) \prec_{N} \cdots \prec_{N} f_{\mathcal{E}}\left(e_{1}\right) \prec_{N} f_{\mathcal{E}}(b)$.

Now assume that $a \in U, b \in D$ and $a \preceq_{M} b$. Then there is an arc $e$ with tail $t_{M}(e)=b$. Hence, $a \preceq_{M} e$. By the previous argument we have $f_{\mathcal{E}}(a) \preceq_{N} f_{\mathcal{E}}(e)$ and Property (F1) implies $f_{\mathcal{E}}(e) \prec_{N} f_{\mathcal{V}}(b)$.

Now assume that $a \in D, b \in U$ and $a \preceq_{M} b$. Then there is an $\operatorname{arc} e$ with tail $h_{M}(e)=a$. Similar arguments as in the previous case show again that $f_{\mathcal{V}}(a) \prec_{N} f_{\mathcal{E}}(b)$.

Finally, assume that $a, b \in D$. If $a=b$, then clearly $f_{\mathcal{V}}(a)=f_{\mathcal{V}}(b)$. So assume $a \neq b$ and, thus, $a \prec_{M} b$. Then, there is an $\operatorname{arc} e$ with $t_{M}(e)=b$ and an $\operatorname{arc} e^{\prime}$ with $h_{M}\left(e^{\prime}\right)=a$. Similar arguments as in the previous cases combined with Property (F1) imply that $f_{\mathcal{V}}(a) \prec_{N} f_{\mathcal{E}}\left(e^{\prime}\right) \preceq_{N} f_{\mathcal{E}}(e) \prec_{N}$ $f_{\mathcal{V}}(b)$. 


\section{Existence of Reconciliation Maps to Networks}

In Fig. 2, we presented an example which shows that if there is a TreeNet-reconciliation map from an event-labeled gene tree $(T ; t, \sigma)$ to a network $N$, then $N$ does not need to display all informative triples in $\mathcal{S}(T ; t, \sigma)$. In fact, the network may display species triples that are not supported by $(T ; t, \sigma)$. For example, the network in Fig. 2 displays the triple $B C \mid A$ although $A B \mid C \in \mathcal{S}(T ; t, \sigma)$. In other words, a network $N$, for which a TreeNet-reconciliation map from $(T ; t, \sigma)$ to $N$ exists, does not need to preserve much (or possibly even any) of the structure of $T$. Thus, the question arises as to whether there always exists a TreeNet-reconciliation map from $(T ; t, \sigma)$ to some network? In Theorem 5 , the main result of this section, we show that this is indeed always the case.

To establish Theorem 5, we first define reconciliation maps between event-labeled gene trees and pseudo-MUL-trees, a topic that has recently been studied in a somewhat different form in [21]. Then, we show how to associate to any event-labeled gene tree $(T ; t, \sigma)$ a MUL-tree $(M(T ; t, \sigma), \chi)$ such that $(T ; t, \sigma)$ can be reconciled with this MUL-tree via some map $\kappa_{(T ; t, \sigma)}$. Using the notion of a "combined reconciliation map", we then exploit $\kappa_{(T ; t, \sigma)}$ to define a reconciliation map between $(T ; t, \sigma)$ and the fold up of $(M(T ; t, \sigma), \chi)$.

Definition 6 (Reconciliation map to a pseudo MUL-tree). Suppose that $\mathbb{S}$ is a set of species, that $M=(D, U)$ is a pseudo MUL-tree on $\mathbb{S}$, and that $(T ; t, \sigma)$ is an event-labeled gene tree on $\mathbb{G}$.

Then, we say that $(M, \chi)$ is a pseudo MUL-tree for $(T=(V, E) ; t, \sigma)$ if there is a map $\kappa: V \rightarrow$ $\left(D \backslash D^{1}\right) \cup U$ such that, for all $x \in V$ :

(M1) Leaf Constraint. If $x \in \mathbb{G}$ then $\kappa(x) \in L(M)$ and $\kappa(x) \in \chi(\sigma(x))$

(M2) Event Constraint.

(i) If $t(x)=\mathfrak{s}$ and $x$ has children $x_{1}, \ldots, x_{k}, k \geq 2$, then $\kappa(x) \in D \backslash L(M)$ and there exists a directed path $P_{i}$ from $\kappa(x)$ to $\kappa\left(x_{i}\right)$ and a directed path $P_{j}$ from $\kappa(x)$ to $\kappa\left(x_{j}\right)$ for two distinct $i, j \in\{1, \ldots, k\}$ such that, in $M$, the first arc on $P_{i}$ is incomparable with the first $\operatorname{arc}$ on $P_{j}$.

(ii) If $t(x)=\mathfrak{d}$, then $\kappa(x) \in U$.

(M3) Ancestor Constraint.

Let $x, y \in V$ with $x \prec_{T} y$, then we distinguish between the two cases:

(i) If $t(x)=t(y)=\mathfrak{d}$, then $\kappa(x) \preceq_{M} \kappa(y)$,

(ii) otherwise, i.e., at least one of $t(x)$ and $t(y)$ is a speciation $\mathfrak{s}, \kappa(x) \prec_{M} \kappa(y)$.

We call $\kappa$ the MUL-reconciliation map from $(T ; t, \sigma)$ to $M$.

Note that Properties (M1), (M2.ii) and (M3) are canonical extensions of the Properties (R1), (R2.ii) and (R3) of TreeNet-reconciliation maps. Moreover, Property (M2.i) and the fact that $M$ has no hybrid vertices implies the following weaker property for all $x \in D$ : if $t(x)=\mathfrak{s}$ and $x$ has at least two children, then there exist two children $x_{1}$ and $x_{2}$ such that $\kappa\left(x_{1}\right)$ and $\kappa\left(x_{2}\right)$ are incomparable in $M$. However, the converse implication does not always hold. Moreover, Property (M2.i) cannot be weakened to establish results for TreeNet-reconciliation maps based on MUL-reconciliation maps and particular foldings.

We next provide a construction that allows us to associate a MUL-tree to an event-labeled gene tree. Suppose $(T ; t, \sigma)$ is an event-labeled gene tree. Let $(M(T ; t, \sigma), \chi)$ denote the MULtree obtained from $(T ; t, \sigma)$ as follows: First replace every leaf of $T$ by its label under $\sigma$. Next, add an incoming arc to the root of $T$ to obtain a tree with root having outdegree 1 . The resulting MUL-tree is $M(T ; t, \sigma)$. To obtain $\chi$, we put $\chi(x)=\{l \in L(T) \mid \sigma(l)=x\}$, for all $x \in \mathbb{S}$.

Definition 7. Suppose that $(T=(V, E) ; t, \sigma)$ is an event-labeled gene tree. Then we call the $M U L-$ tree $(M(T ; t, \sigma), \chi)$ the MUL-tree for $(T ; t, \sigma)$. Furthermore, putting $M(T ; t, \sigma)=(D, U)$ we refer 
to the map $\kappa_{(T ; t, \sigma)}: V \rightarrow D \cup U$ given by putting $\kappa_{(T ; t, \sigma)}(x)=\sigma(x)$ if $x \in V$ and, for all $x \in V^{0}$, by putting $\kappa_{(T ; t, \sigma)}(x)=x$ if $t(x)=\mathfrak{s}$ and $\kappa_{(T ; t, \sigma)}(x)=e^{x}$ otherwise as the (trivial) map from $(T ; t, \sigma)$ to $(M(T ; t, \sigma), \chi)$

Lemma 7. The map $\kappa_{(T ; t, \sigma)}$ is a MUL-reconciliation from $(T ; t, \sigma)$ to $(M(T ; t, \sigma), \chi)$.

Proof. For simplicity let $M:=M(T ; t, \sigma)$. By definition, $\kappa:=\kappa_{(T ; t, \sigma)}$ satisfies (M1), (M2.ii) and (M3). To see that $\kappa$ is a MUL-reconciliation map, it thus remains to show that Property (M2.i) is satisfied. Let $x$ be a vertex in $T$ with $t(x)=\mathfrak{s}$ and with children $x_{1}, \ldots, x_{k}, k \geq 2$. By definition, $\kappa(x) \in D \backslash L(M)$. Now let $x_{i}$ and $x_{j}$ be two distinct children of $x$. By construction of $M$, all vertices of $T$ are contained in $M$. However, to make the reading easier, we denote by $v^{\prime}$ the vertices in $M$ that correspond to vertex $v$ in $T$. Each of the two vertices $x_{i}$ and $x_{j}$ may be a leaf or an inner vertex equipped with a particular event $\mathfrak{s}$ or $\mathfrak{d}$. A straight-forward case analysis and the fact that $\kappa\left(x_{l}\right)$ is either $x_{l}^{\prime}$ or the arc $e^{x_{l}^{\prime}}$ where $l \in\{i, j\}$ together with Property (M3) shows that in either case we have $x_{i}^{\prime} \preceq_{M} \kappa\left(x_{i}\right) \prec_{M} \kappa(x)$ and $x_{j}^{\prime} \preceq_{M} \kappa\left(x_{j}\right) \prec_{M} \kappa(x)$. Note that, by construction, the path from $\kappa(x)$ to $\kappa\left(x_{i}\right)$ is the arc $e^{x_{i}^{\prime}}$ and the path from $\kappa(x)$ to $\kappa\left(x_{j}\right)$ is the arc $e^{x_{j}^{\prime}}$. Since, $M$ is a tree and $x_{i}$ and $x_{j}$ are incomparable in $T$ the vertices $x_{i}^{\prime}$ and $x_{j}^{\prime}$ as well as the arcs $e^{x_{i}^{\prime}}$ and $e^{x_{j}^{\prime}}$ must be incomparable in $M$. Therefore, (M2.i) is also satisfied.

Calling the map $\kappa_{(T ; t, \sigma)}$ the trivial MUL-reconciliation map, we now prove a technical result which will allow us to link trivial MUL-reconciliation maps with foldings.

Lemma 8. Suppose that $(T ; t, \sigma)$ is an event-labeled gene tree and that $(M, \chi)$ is a MUL-tree. If there is a MUL-reconciliation map from $(T ; t, \sigma)$ to $(M, \chi)$, then there is a MUL-reconciliation map from $(T ; t, \sigma)$ to any subdivision $\left(M^{\prime}, \chi\right)$ of $(M, \chi)$.

Proof. Let $\kappa$ be a MUL-reconciliation map from an event-labeled gene tree $(T=(V, E) ; t, \sigma)$ to some MUL-tree $(M=(D, U), \chi)$ and $\left(M^{\prime}, \chi\right)$ be a subdivision of $(M, \chi)$. For each $\operatorname{arc} e=(u, v)$ of $M$ that is subdivided in the construction of $M^{\prime}$ by a directed path $P_{u v}$ from $u$ to $v$ in $M^{\prime}$ we denote by $e^{*}$ the last arc in $P_{u v}$. If $e$ is not subdivided, we put $e^{*}=e$. For all $v \in V$ put

$$
\kappa^{\prime}(v)= \begin{cases}\kappa(v) & \text { if } \kappa(v) \in D \\ \kappa(v)^{*} & \text { else. }\end{cases}
$$

It is now straight-forward to see that $\kappa^{\prime}$ is a MUL-reconciliation map from $(T ; t, \sigma)$ to $\left(M^{\prime}, \chi\right)$.

We are now in the position to establish the main result of this section.

Theorem 5. Given a MUL-reconciliation map $\kappa$ from an event-labeled gene tree $(T=(V, E) ; t, \sigma)$ to a pseudo $M U L$-tree $(M, \chi)$, and a folding map $f$ from $(M=(D, U), \chi)$ to a network $N=(W, F)$. Then, the map $\mu_{\kappa, f}: V \rightarrow W \cup F$ defined by putting for every $v \in V$

$$
\mu_{\kappa, f}(v)= \begin{cases}f_{\mathcal{V}}(\kappa(v)) & \text { if } \kappa(v) \in D, \\ f_{\mathcal{E}}(\kappa(v)) & \text { otherwise, }\end{cases}
$$

is a TreeNet-reconciliation map from $(T ; t, \sigma)$ and $N$.

Proof. We need to show that $\mu:=\mu_{\kappa, f}$ satisfies Properties (R1) $-(\mathrm{R} 3)$.

To see Property (R1), let $x \in \mathbb{G}$. Since Property (M1) is satisfied for $\kappa$ we have $\kappa(x) \in L(M)$ and $\kappa(x) \in \chi(\sigma(x))$. By Property (F2) and the construction of $\mu$, we have $\mu(x)=f_{\mathcal{V}}(\kappa(x))=$ $\sigma(x)$. Thus, Property (R1) holds.

To see Property (R2.i), let $x \in V$ be a vertex with $t(x)=\mathfrak{s}$ and children $x_{1}, \ldots, x_{k}, k \geq 2$. We need to show that $\mu(x) \in Q_{N}^{2}\left(\mu\left(x_{1}\right), \ldots, \mu\left(x_{k}\right)\right)$. By (M2.i), there exists a directed path $P_{i}$ from 
(1)

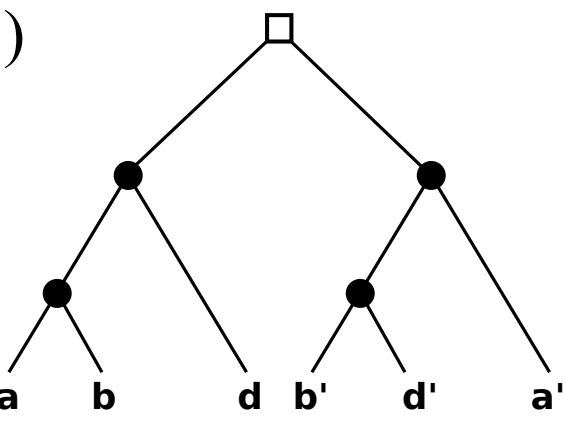

(3)

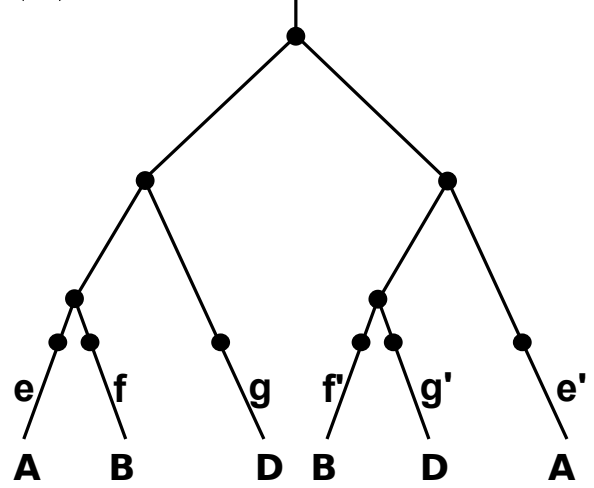

(2)

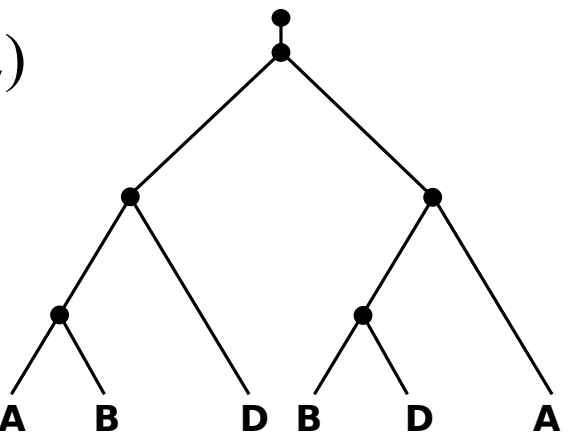

(4)

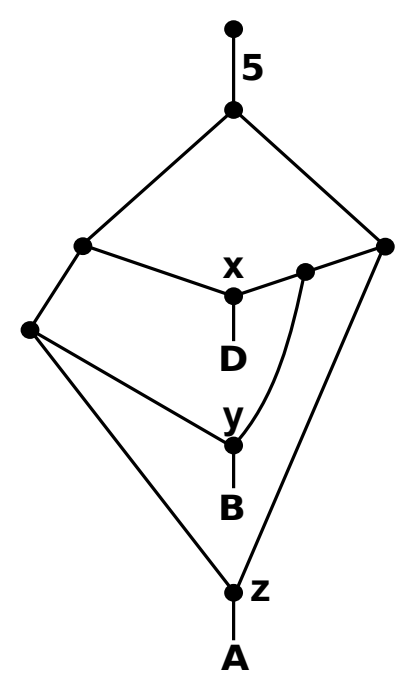

Figure 5: In Panel (1), we depict an event-labeled gene tree $(T ; t, \sigma)$. Speciation and duplication events are represented as $\bullet$ and $\square$, respectively. The corresponding MUL-tree $(M(T ; t, \sigma), \chi)$ and the simple subdivision $\left(M^{\prime}, \chi\right)$ of $(M(T ; t, \sigma), \chi)$ are shown in Panels (2) and (3), respectively. As shown in the proof of Lemma 5, $\left(M^{\prime}, \chi\right)$ can be folded onto $N$ (Panel (4)). Here, $N$ is obtained from $\left(M^{\prime}, \chi\right)$ by identifying the $\operatorname{arcs} e$ and $e^{\prime}$ that yield the $\operatorname{arc}(z, A)$ in $N$, the $\operatorname{arcs} f$ and $f^{\prime}$ that yield the arc $(y, B)$ in $N$, and the $\operatorname{arcs} g$ and $g^{\prime}$ that yield the $\operatorname{arc}(x, D)$ in $N$. By Definition 4 and since $M^{\prime}$ is a subdivision of $M(T ; t, \sigma)$, the MUL-tree $(M(T ; t, \sigma), \chi)$ can be folded onto $N$. 
$\kappa(x)$ to $\kappa\left(x_{i}\right)$ in $M$ and a directed path $P_{j}$ from $\kappa(x)$ to $\kappa\left(x_{j}\right)$ in $M$ for two distinct $i, j \in\{1, \ldots, k\}$ such that in $M$ the first arc on $P_{i}$ is incomparable with the first $\operatorname{arc}$ on $P_{j}$. Let $a_{i}$ and $a_{j}$ be the first $\operatorname{arc}$ on $P_{i}$ and $P_{j}$, respectively. Hence, $t_{M}\left(a_{i}\right)=t_{M}\left(a_{j}\right)=\kappa(x)$ and, since $f$ is a folding map, Property (F1) implies that $t_{N}\left(f_{\mathcal{E}}\left(a_{i}\right)\right)=t_{N}\left(f_{\mathcal{E}}\left(a_{j}\right)\right)=f_{\mathcal{V}}(\kappa(x))$. The latter together with (F1) implies that $a_{i}$ and $a_{j}$ are mapped in $N$ to either the same arc or to two distinct arcs that share the same tail.

Assume first that $f_{\mathcal{E}}\left(a_{i}\right)=f_{\mathcal{E}}\left(a_{j}\right)=e$ and put $v=\kappa(x)$. Then, $f_{\mathcal{V}}(v)=t_{N}(e)$ and $\widetilde{a} \in\left\{a_{i}, a_{j}\right\}$ is an $\operatorname{arc}$ in $M$ that satisfies $f_{\mathcal{E}}(\widetilde{a})=e$ and $t_{M}(\widetilde{a})=v$; contradicting Property (F3). Therefore, $a_{i}$ and $a_{j}$ must be mapped in $N$ to two distinct arcs $e_{i}$ and $e_{j}$, respectively, that share the same tail. Since, $e_{i} \neq e_{j}$ and $t_{N}\left(e_{i}\right)=t_{N}\left(e_{j}\right)=f_{\mathcal{V}}(\kappa(x))$, the arcs $e_{i}$ and $e_{j}$ are incomparable in $N$. Combined with Property (M3) and Lemma 6 it follows that there is a path $P_{i}^{\prime}$ from $\mu(x)$ to $\mu\left(x_{i}\right)$ in $N$ that contains $e_{i}$ and a path $P_{j}^{\prime}$ from $\mu(x)$ to $\mu\left(x_{j}\right)$ in $N$ that contains $e_{j}$. Thus, $\mu\left(x_{i}\right)$ and $\mu\left(x_{j}\right)$ are separated by $\mu(x)$. Therefore, $\mu(x) \in Q_{N}^{2}\left(\mu\left(x_{1}\right), \ldots, \mu\left(x_{k}\right)\right)$.

Clearly, Property (R2.ii) follows from the fact that $\kappa$ satisfies (M2.ii), that $f_{\mathcal{E}}$ maps an arc of $M$ to an $\operatorname{arc}$ of $N$ and the construction of $\mu$.

It remains to show that Property (R3) is satisfied. Suppose that $x, y \in V$ with $x \prec_{T} y$. Clearly, $x \neq y$. If $t(x)=t(y)=\mathfrak{d}$, then $\kappa(x) \preceq_{M} \kappa(y)$ in view of Property (M3.i). Lemma 6 implies that $\mu(x) \preceq_{N} \mu(y)$. Thus, Property (R3.i) is satisfied. Now assume that at least one of $t(x)$ or $t(y)$ is a speciation vertex. Property (M3.ii) implies that $\kappa(x) \prec_{M} \kappa(y)$. Note that not both of $\kappa(x)$ and $\kappa(y)$ can be contained in $U$. Again, Lemma 6 implies that $\mu(x) \prec_{N} \mu(y)$. Therefore, (R3.ii) is also satisfied by $\mu$.

In summary, it follows that $\mu$ is a TreeNet-reconciliation map from $(T ; t, \sigma)$ to $N$.

Corollary 3. Given any event-labeled gene tree $(T ; t, \sigma)$, there exists a species network $N$ for $(T ; t, \sigma)$. Moreover, the network $N$ as well as the TreeNet-reconciliation map from $(T ; t, \sigma)$ to $N$ can be constructed in polynomial time.

Proof. Let $\kappa_{(T ; t, \sigma)}$ be the trivial MUL-reconciliation from $(T ; t, \sigma)$ to $(M, \chi)$ with $M:=M(T ; t, \sigma)$ (which is a reconciliation map by Lemma 7). Moreover, let $\left(M^{\prime}, \chi\right)$ be the simple subdivision of $(M, \chi)$. Let $N$ be the network as constructed in the proof of Lemma 5 and let $f$ denote the underlying folding map from $M^{\prime}$ to $N$. Lemma 8 implies that there is a reconciliation map $\kappa^{\prime}$ from $(T ; t, \sigma)$ to $\left(M^{\prime}, \chi\right)$. Then, by Theorem 5, the composed reconciliation map $\mu_{\mathcal{K}^{\prime}, f}$ is a reconciliation map from $(T ; t, \sigma)$ to $N$. Hence, $N$ is a species network for $(T ; t, \sigma)$.

Finally, Lemma 5 implies that the network $N$ and the folding map $f$ can be constructed in polynomial time. Furthermore, the construction of $\kappa$ and thus, of $\kappa^{\prime}$ as well as of $\mu_{\kappa^{\prime}, f}$ can be done in polynomial time. This proves the second part of the corollary.

\section{Existence of Reconciliations for Multi-Arc Free Net- works}

In the last section, we showed that every event-labeled gene tree can be reconciled with some network. An important assumption in this result is that the network is permitted to contain multiarcs. Although not unreasonable, in practice (and in much of the literature on networks) it can be desirable to restrict attention to networks which do not have multi-arcs.

Definition 8. An event-labeled gene tree $(T ; t, \sigma)$ is well-behaved if given any $v \in V$ with $t(v)=\mathfrak{s}$, for every child $v^{\prime}$ of $v$ in $T$ there is another child $v^{\prime \prime}$ of $v$ in $T$ with $\sigma\left(L_{T}\left(v^{\prime}\right)\right) \neq \sigma\left(L_{T}\left(v^{\prime \prime}\right)\right)$.

Equivalently, an event-labeled gene tree $(T ; t, \sigma)$ is well-behaved if for all speciation vertices $v$ in $T, \sigma\left(L_{T}\left(v_{1}\right)\right)=\sigma\left(L_{T}\left(v_{2}\right)\right)=\cdots=\sigma\left(L_{T}\left(v_{k}\right)\right)$ does not hold for $v_{1}, v_{2} \ldots v_{k}, k \geq 2$, the children of $v$. The latter is a reasonable and a quite weak restriction as in many applications the stronger 
condition $\sigma\left(L_{T}\left(v^{\prime}\right)\right) \cap \sigma\left(L_{T}\left(v^{\prime \prime}\right)\right)=\emptyset$ for any two distinct children $v^{\prime}$ and $v^{\prime \prime}$ of a speciation vertex is (at least implicitly) required; see e.g. [26, 34, 35, 41, 56]. In this section, we show that for every well-behaved event-labeled gene tree $(T ; t, \sigma)$ there is a TreeNet-reconciliation map to some network $N$ without multi-arcs and that, in addition, $N$ displays all triples in $\mathcal{S}(T ; t, \sigma)$.

To this end, we will first take the folding of the simple subdivision $\left(M^{\prime}, \chi\right)$ of $(M(T ; t, \sigma), \chi)$ to obtain a network $N$, as specified in Definition 5 and the proof of Lemma 5. However, this network may contain multi-arcs (see Fig. 4). To adjust for this, one may be tempted to simply remove multi-arcs and subsequently suppress degree two vertices. This, however, can be problematic as it may result in a network $N^{\prime}$ for which no folding map from $M^{\prime}$ to $N^{\prime}$ exists. For example, consider the network $N$ depicted in Fig. 4 (right). The removal of one of the arcs between $y$ and $b$, yields a graph which is not a network (since then vertex $y$ has in- and outdegree one). Additional suppression of $y$ results in a network $N^{\prime}$, for which no folding map from the pseudo MUL-tree $M^{\prime}$ pictured in Fig. 4 (center) exists, since Property (F1) is violated for the resulting arc in $N$ and vertex $b$ of $M^{\prime}$. Since there is no folding map from $\left(M^{\prime}, \chi\right)$ to $N^{\prime}$, we can cannot apply Theorem 5 to conclude that there is a TreeNet-reconciliation map from $(T ; t, \sigma)$ to $N^{\prime}$ (if there is one).

In order to obtain a multi-arc free network from $N$ for which there is a TreeNet-reconciliation from $(T ; t, \sigma)$, we next provide an alternative approach. For this, we need to define two sets which will turn out to be helpful in the construction of multi-arc free networks from networks that may contain multi-arcs.

Definition 9. Let $N=(W, F)$ be a network on $\mathbb{S}$. For each $x \in \mathbb{S}$, we denote by $\mathcal{W}_{x}^{N}$ the inclusionmaximal subset of vertices of $W$ that comprises all vertices $v \in W$ that satisfy $x \prec_{N} v$ and $L_{N}(v)=$ $\{x\}$.

Moreover, we define $\mathcal{V}_{x}^{N} \subseteq W$ as the set of all vertices $z \in W$ with $z \notin \mathcal{W}_{x}^{N}$ and there is an arc $(z, w)$ in $N$ with $w \in \mathcal{W}_{x}^{N}$.

To illustrate theses two sets consider the bottom left network $N$ depicted in Fig. 6. Then $\mathcal{W}_{x}^{N}$ consists of all vertices $v$ in $N$ that are highlighted by colored " $\star$ " and that satisfy $v \succ_{N} x$ for the particular leaf $x \in \mathbb{S}$. Thus, the set $W_{1}=W \backslash \cup_{x \in \mathbb{S}^{\prime}}\left(\mathcal{W}_{x}^{N} \cup\{x\}\right)$ where $\mathbb{S}^{\prime}$ denotes the set of all $x \in \mathbb{S}$ with $\mathcal{W}_{x}^{N} \neq \emptyset$ is the set $W_{1}=\{1,2,3,4\}$.

Lemma 9. Let $N=(W, F)$ be a network on $\mathbb{S}$. Then, the following statements are satisfied.

(i) $x \notin \mathcal{W}_{x}^{N}$, for all $x \in \mathbb{S}$.

(ii) For all $x \in \mathbb{S}$, if $v \in \mathcal{W}_{x}^{N}$, then every vertex $u \in W$ with $u \prec_{N} v$ and $u \neq x$ must be contained in $\mathcal{W}_{x}^{N}$.

(iii) There are no arcs between $\mathcal{W}_{x}^{N}$ and $\mathcal{W}_{y}^{N}$ and $\mathcal{W}_{x}^{N} \cap \mathcal{W}_{y}^{N}=\emptyset$, for all $x, y \in \mathbb{S}$ distinct.

(iv) If $\mathcal{W}_{x}^{N} \neq \emptyset$, then $\mathcal{V}_{x}^{N} \neq \emptyset$

Proof. Property (i) is trivially satisfied, since $x \nprec_{N} x$.

For Property (ii), assume for contradiction that there exists some $v \in \mathcal{W}_{x}^{N}$ and some vertex $u \in W$ with $u \prec_{N} v$ and $u \neq x$ that is not contained in $\mathcal{W}_{x}^{N}$. Then $L_{N}(u) \neq\{x\}$ and since $v \succ_{N} u$ also $L_{N}(v) \neq\{x\}$; a contradiction.

We continue with Property (iii). In view of Lemma 9(ii), there are no $\operatorname{arcs}(u, v) \in F$ with $u \in \mathcal{W}_{x}^{N}$ and $v \notin \mathcal{W}_{x}^{N}$ and thus, in particular, no $\operatorname{arcs}(u, v) \in F$ with $u \in \mathcal{W}_{x}^{N}$ and $v \in \mathcal{W}_{y}^{N}$ for all distinct $x, y \in \mathbb{S}$. By construction, $\mathcal{W}_{x}^{N} \cap \mathcal{W}_{y}^{N}=\emptyset$, for all distinct $x, y \in \mathbb{S}$.

For Property (iv), assume for contradiction that $\mathcal{W}_{x}^{N} \neq \emptyset$ but $\mathcal{V}_{x}^{N}=\emptyset$. Hence, there is no arc $(z, w) \in F$ with $z \notin \mathcal{W}_{x}^{N}$ and $w \in \mathcal{W}_{x}^{N}$. Thus, $\rho_{N} \in \mathcal{W}_{x}^{N}$; a contradiction to $\rho_{N} \succ_{N} z$ for all $z \in \mathbb{S}$ and $|\mathbb{S}|>1$.

In what follows, we wish to modify a network $N$ with multi-arcs to a network $N^{*}$ without multi-arcs. To this end, we will replace entire subgraphs of $N$ by specified arcs or vertices which eventually leads to the multi-arc free network $N^{*}$. We give a formal description of the approach 

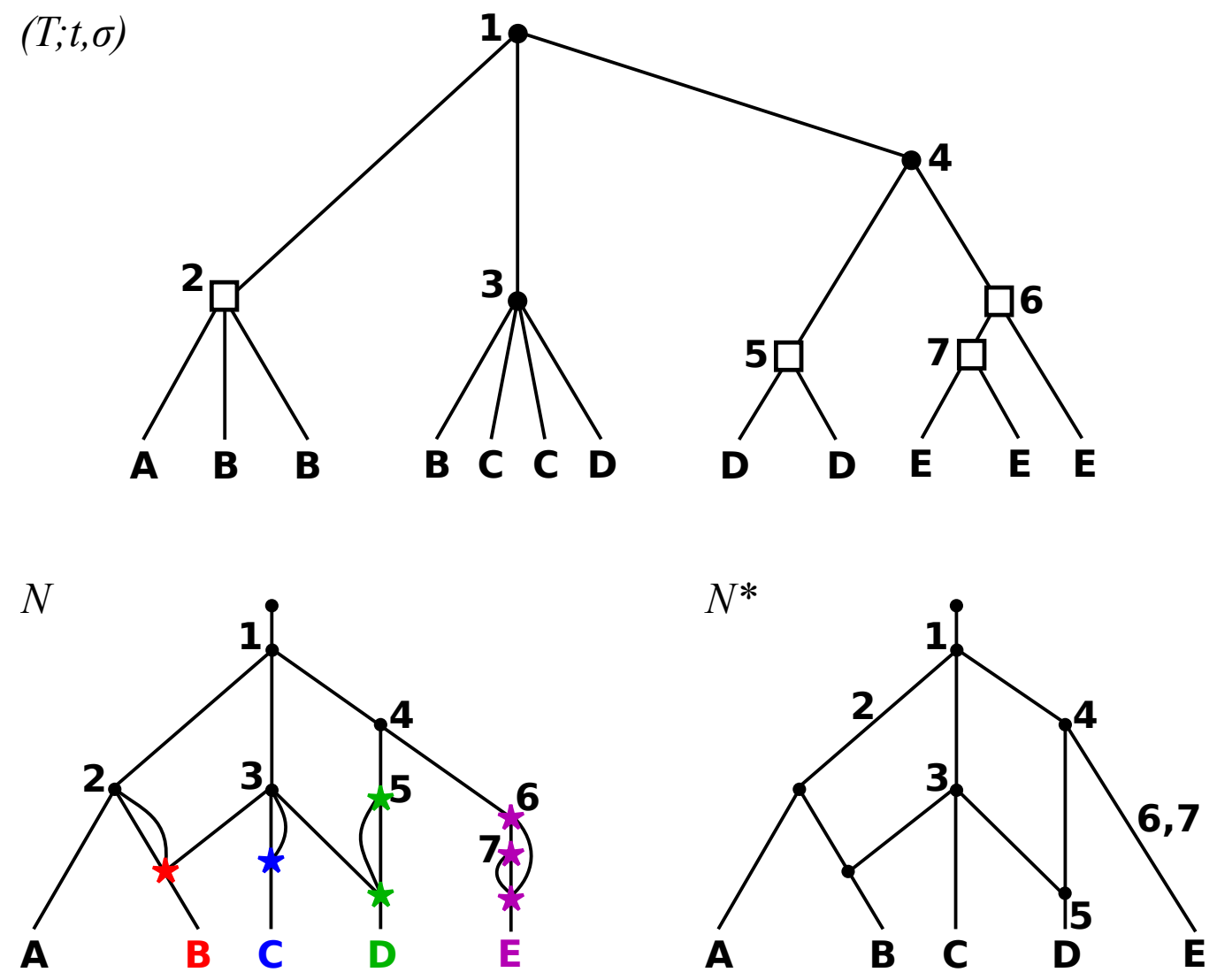

Figure 6: In the upper part, an event-labeled gene tree $(T ; t, \sigma)$ is shown where, for simplicity, all leaf labels $v \in \mathbb{G}$ are replaced by $\sigma(v) \in \mathbb{S}=\{A, B, C, D, E\}$. Speciation and duplication events are represented as $\bullet$ and $\square$, respectively. In the lower-left part, the network $N=(W, F)$ obtained from the simple subdivision $\left(M^{\prime}, \chi\right)$ of $(M(T ; t, \sigma) ; \chi)$ as in Definition 5 (ignoring the " $\star$ "-labels of the non-leaf vertices). In the lower-right part, the network $N^{*}$ constructed from $N$ as specified in Definition 10. Using the vertex labels in $(T ; t, \sigma)$, we indicate the TreeNet-reconciliation map $\mu^{*}$ from $(T ; t, \sigma)$ to $N^{*}$ as specified in the proof of Proposition 4 in terms of the $\mu^{*}$-images on the respective vertices and arcs in $N^{*}$. 
to first construct a DAG $N^{*}$ from a given network $N$. As we shall see in Proposition 3, this DAG $N^{*}$ is indeed a multi-arc free network.

Definition 10. Let $N=(W, F)$ be a network on $\mathbb{S}$. The DAG $N^{*}$ is obtained from $N$ as follows:

First, for all $x \in \mathbb{S}$ with $\mathcal{W}_{x}^{N} \neq \emptyset$ and $\left|\mathcal{V}_{x}^{N}\right|=1$, remove all vertices in $\mathcal{W}_{x}^{N}$ and all arcs in $F$ incident to vertices in $\mathcal{W}_{x}^{N}$ from $N$ and add the arc $(z, x)$ to $N$ with $z \in \mathcal{V}_{x}^{N}$.

Second, for all $x \in \mathbb{S}$ with $\mathcal{W}_{x}^{N} \neq \emptyset$ and $\left|\mathcal{V}_{x}^{N}\right|>1$, remove all vertices in $\mathcal{W}_{x}^{N}$ and all arcs in $F$ incident to vertices in $\mathcal{W}_{x}^{N}$ from $N$ and add a new vertex $w_{x}$ and one arc $\left(z, w_{x}\right)$ for all $z \in \mathcal{V}_{x}^{N}$ and the $\operatorname{arc}\left(w_{x}, x\right)$ to $N$.

The sets $\mathcal{W}_{x}^{N}$ and $\mathcal{V}_{x}^{N}$ as well as the construction of $N^{*}$ are illustrated in Fig. 6.

Proposition 3. Let $(T ; t, \sigma)$ be an event-labeled gene tree on $\mathbb{G}$ and let $N$ be a species network on $\mathbb{S}$ obtained from the simple subdivision of the MUL-tree $(M(T ; t, \sigma), \chi)$ as in Definition 5. Then $N^{*}$ obtained from $N$ by Def. 10 is a multi-arc free species network on $\mathbb{S}$ and the construction of $N^{*}$ can be done in polynomial time.

Proof. In what follows, let $T=(V, E), N=(W, F)$ and $M(T ; t, \sigma)=(D, U)$. To help keep notation at bay, we assume for simplicity that $V=D \backslash\left(\left\{\rho_{M(T ; t, \sigma)}\right\} \cup \mathbb{G}\right)$.

Now, we construct the DAG $N^{*}$ from $N$ as in Definition 10. Clearly, $N^{*}$ has leaf set $\mathbb{S}$. To show that $N^{*}$ is a multi-arc free network, we first analyze for some $x \in \mathbb{S}$ the sets $\mathcal{W}_{x}^{N}$ and $\mathcal{V}_{x}^{N}$. Note, if $\mathcal{W}_{x}^{N} \neq \emptyset$, then Lemma 9(iii) implies that $\mathcal{V}_{x}^{N} \neq \emptyset$, Thus, we examine the following three mutually exclusive cases:

(I) $\mathcal{W}_{x}^{N}=\emptyset$,

(II) $\mathcal{W}_{x}^{N} \neq \emptyset$ and $\left|\mathcal{V}_{x}^{N}\right|=1$, or

(III) $\mathcal{W}_{x}^{N} \neq \emptyset$ and $\left|\mathcal{V}_{x}^{N}\right|>1$.

We interrupt the proof of the proof of the proposition to illustrate these cases by means of the bottom left network in Fig. 6 . Then the set $\mathcal{W}_{A}^{N}=\emptyset$ satisfies Case (I). For $B, D \in \mathbb{S}$, we have $\mathcal{W}_{B}^{N} \neq \emptyset$ and $\mathcal{V}_{B}^{N}=\{2,3\}$ and $\mathcal{W}_{D}^{N} \neq \emptyset$ and $\mathcal{V}_{D}^{N}=\{3,4\}$ and hence, Case (III) is satisfied. For $C, E \in \mathbb{S}$, we have $\mathcal{W}_{C}^{N} \neq \emptyset$ and $\mathcal{V}_{C}^{N}=\{3\}$ and $\mathcal{W}_{E}^{N} \neq \emptyset$ and $\mathcal{V}_{E}^{N}=\{4\}$ and hence, Case (II) is satisfied.

We continue with the proof of the proposition by taking a closer look at potential multi-arcs in $N$. By construction of $N$ and since $(T ; t, \sigma)$ has no multi-arcs, there are exactly $\ell \geq 2$ multi-arcs between some vertices $u$ and $v$ in $N$ with $v \prec_{N} u$ if and only if there are exactly $\ell \operatorname{arcs} e_{1}, \ldots, e_{\ell}$ incident to $u$ in $(T ; t, \sigma)$ with $\sigma\left(h_{T}\left(e_{i}\right)\right)=x, 1 \leq i \leq \ell$. Put differently, if there are multi-arcs between two vertices $u$ and $v$ in $N$ with $v \prec_{N} u$, then, we have in $N$ that $v=\operatorname{par}(x)$ for some $x \in \mathbb{S}$ and the outdegree of $\operatorname{par}(x)$ is one. In particular, $\operatorname{par}(x) \in \mathcal{W}_{x}^{N}$ which implies $\mathcal{W}_{x}^{N} \neq \emptyset$. We summarize the latter observation for

Case (I): Let $x \in \mathbb{S}$ such that $\mathcal{W}_{x}^{N}=\emptyset$. Then the construction of $N$ from $M(T ; t, \sigma)$ implies that there are no multi-arcs in $N$ between the (unique) parent $\operatorname{par}(x)$ of $x$ and any of the vertices $y$ that are parents of $\operatorname{par}(x)$. Therefore, if $\mathcal{W}_{x}^{N}=\emptyset$ for all $x \in \mathbb{S}$, then $N$ is a network without multi-arcs. In this case, we put $N^{*}=N$.

Case (II): Let $x \in \mathbb{S}$ such that $\mathcal{W}_{x}^{N}=\left\{w_{1}, \ldots, w_{n}\right\}, n \geq 1$, and $\mathcal{V}_{x}^{N}=\{z\}$. According to Definition 10, we remove all vertices in $\mathcal{W}_{x}^{N}$ and all arcs in $F$ incident to vertices in $\mathcal{W}_{x}^{N}$ from $N$ and add the arc $(z, x)$ to $N$ to obtain an acyclic digraph $N^{\prime}=\left(W^{\prime}, F^{\prime}\right)$. By Lemma 9(ii), there is no $\operatorname{arc}\left(w_{i}, u\right)$ in $N$ with $u \notin \mathcal{W}_{x}^{N}$ and $u \neq x$. Thus, the ancestor relationship between any vertices in $N$ that are still contained in $N^{\prime}$ (and thus, not contained in $\mathcal{W}_{x}^{N}$ ) is preserved. Put differently, if $u, v \in W \cap W^{\prime}$ with $u \preceq_{N} v$ then $u \preceq_{N^{\prime}} v$.

We next show that $N^{\prime}$ is a network and thus, satisfies Properties (N1), (N2) and (N3). By construction, Property (N1) is clearly satisfied for $N^{\prime}$. Furthermore, Property (N2) clearly holds for 
all $y \in \mathbb{S} \backslash\{x\}$. Since all vertices $\mathcal{W}_{x}^{N}$ have been removed and only the arc $e^{x}$ has been added, vertex $x$ is an outdegree- 0 vertex in $N^{\prime}$. Therefore, $N^{\prime}$ satisfies Property (N2).

To see Property (N3), observe first that the degrees of the vertices in $N^{\prime}$ that are not incident to vertices in $\mathcal{W}_{x}^{N}$ remain the same as in $N$. Thus, $N^{\prime}$ satisfies Property (N3) for all such vertices. Moreover, since all vertices $\mathcal{W}_{x}^{N}$ have been removed and only the arc $e^{x}$ has been added, it remains to analyze the degree of the vertex $z:=h_{N}\left(e^{x}\right)$ in $N^{\prime}$. In the context of this, we claim that $z$ is a tree vertex of $N^{\prime}$. To see this, note that in $N$, vertex $z$ cannot have indegree greater than one, as otherwise, the outdegree of $z$ must be one in $N$ and thus, $z \in \mathcal{W}_{x}^{N}$; a contradiction. Thus, the indegree of $z$ is one in $N$ and, by construction, the indegree of $z$ remains one in $N^{\prime}$. Since $N$ satisfies (N3), the outdegree of $z$ is greater than one in $N$. However, not all children of $z$ in $N$ can be contained in $\mathcal{W}_{x}^{N}$ as otherwise, $z \in \mathcal{W}_{x}^{N}$; a contradiction. Thus, there is a child $z^{\prime}$ of $z$ with $z^{\prime} \notin \mathcal{W}_{x}^{N}$. Since the removal of $\mathcal{W}_{N}^{x}$ and the respective incident arcs as well as the addition of the $\operatorname{arc} e^{x}$ does not affect any arc between $z$ and and its children $z^{\prime}$ with $z^{\prime} \notin \mathcal{W}_{x}^{N}$, it follows that the $\operatorname{arc}(\mathrm{s})$ between $z$ and $z^{\prime}$ remain in $N^{\prime}$. Thus, $z$ still has outdegree greater than one in $N^{\prime}$. Moreover, there is no vertex $w_{i} \in \mathcal{W}_{x}^{N}$ with $w_{i} \succ_{N} z$, as otherwise, Lemma 9(ii) implies that $z \in \mathcal{W}_{x}^{N}$; a contradiction. Hence, the unique $\operatorname{arc}(\operatorname{par}(z), z)$ has not been removed and still exists in $N^{\prime}$. Therefore, $z$ has indegree one in $N^{\prime}$. Consequently, $z$ is a tree vertex in $N^{\prime}$, as claimed. In summary, $N^{\prime}$ satisfies (N1)-(N3) and thus, remains a network.

By construction and the latter arguments, $x$ has exactly one parent $\operatorname{par}(x)=z$ in $N^{\prime}$ and $z$ has at least one child $z^{\prime}$ with $z^{\prime} \notin \mathcal{W}_{x}^{N}$ and this child $z^{\prime}$ remains in $N^{\prime}$. Therefore, $\mathcal{W}_{x}^{N^{\prime}}=\emptyset$ in $N^{\prime}$. In addition, Lemma 9(iii) implies that $\mathcal{W}_{a}^{N} \cap \mathcal{W}_{b}^{N}=\emptyset$ and that there are no arcs between $\mathcal{W}_{a}^{N}$ and $\mathcal{W}_{b}^{N}$ for all distinct $a, b \in \mathbb{S}$. Therefore, $\mathcal{W}_{a}^{N^{\prime}}=\mathcal{W}_{a}^{N}$ for all $a \in \mathbb{S} \backslash\{x\}$ in $N^{\prime}$. Put differently, the sets $\mathcal{W}_{a}^{N}$ remain unchanged in $N^{\prime}$ for all $a \in \mathbb{S} \backslash\{x\}$.

Case (III): Let $x \in \mathbb{S}$ such $\mathcal{W}_{x}^{N} \neq \emptyset$ and $\left|\mathcal{V}_{x}^{N}\right|>1$. According to Definition 10, we remove all vertices in $\mathcal{W}_{x}^{N}$ and all arcs in $F$ incident to vertices in $\mathcal{W}_{x}^{N}$ from $N$ and add a new vertex $w_{x}$ and one $\operatorname{arc}\left(z, w_{x}\right)$ for all $z \in \mathcal{V}_{x}^{N}$ and the $\operatorname{arc}\left(w_{x}, x\right)$ to $N$ to obtain an acyclic digraph $N^{\prime}$. Note that, as in Case (II), the ancestor relationship between any two vertices in $N$ that are also contained in $N^{\prime}$ is preserved.

We show next that $N^{\prime}$ is a network. By construction, Properties (N1) and (N2) are satisfied for $N^{\prime}$. It remains to show that $N^{\prime}$ satisfies Property (N3). Since all vertices $\mathcal{W}_{x}^{N}$ have been removed and new arcs have been added only between vertices in $\mathcal{V}_{x}^{N}, w_{x}$ and $x$, we can conclude that if $z$ is not adjacent with a vertex in $\mathcal{W}_{x}^{N}$, then the indegree and outdegree of $z$ in $N$ is the same as the indegree and outdegree of $z$ in $N^{\prime}$. So assume that $z \in \mathcal{V}_{x}^{N} \cup\left\{w_{x}\right\}$.

Assume first that $z \in \mathcal{V}_{x}^{N}$. We claim again that $z$ is a tree vertex of $N^{\prime}$. As observed in Case (II), any vertex $z \in \mathcal{V}_{x}^{N}$ must have indegree one in $N$ and there is a child $z^{\prime}$ with $z^{\prime} \notin \mathcal{W}_{x}^{N}$. The construction of $N^{\prime}$ does not affect any arc between $z$ and $z^{\prime}$. Thus, every arc between $z$ and a child $z^{\prime} \notin \mathcal{W}_{x}^{N}$ is also an arc in $N^{\prime}$. Combined with the fact that, by construction, we have added the $\operatorname{arc}\left(z, w_{x}\right)$, it follows that $z \in \mathcal{V}_{x}^{N}$ has outdegree greater than one in $N^{\prime}$. Moreover, there is no vertex $w_{i} \in \mathcal{W}_{x}^{N}$ with $w_{i} \succ_{N} z$, as otherwise, Lemma 9(ii) implies that $z \in \mathcal{W}_{x}^{N}$; a contradiction. Hence, the unique $\operatorname{arc}(\operatorname{par}(z), z)$ has not been removed and is also an $\operatorname{arc} N^{\prime}$. Therefore, $z$ has indegree one in $N^{\prime}$. Thus, $z$ is a tree vertex in $N^{\prime}$, as claimed.

Finally, assume that $z=w_{x}$. Then, by construction, the indegree of $z$ in $N^{\prime}$ is $\left|\mathcal{V}_{x}^{N}\right|>1$ in $N^{\prime}$ and the unique child of $z$ is $x$. Thus, $z$ is a hybrid vertex in $N^{\prime}$. In summary, $N^{\prime}$ satisfies (N1)-(N3) and thus, is a network.

It is easy to see that $\mathcal{W}_{x}^{N^{\prime}}=\left\{w_{x}\right\}$, where $w_{x}$ is not contained in any multi-arcs. Note also that, as in Case (II), we have $\mathcal{W}_{a}^{N}=\mathcal{W}_{a}^{N^{\prime}}$ for all $a \in \mathbb{S} \backslash\{x\}$.

In the latter construction, we modified $N$ in Cases (II) and (III) for a specific vertex $x \in \mathbb{S}$ to obtain a network $N^{\prime}$.

We complete the proof by associating a species network $N^{*}$ to $N$ as follows. Bearing in mind Case (I), we first apply to all vertices $x \in \mathbb{S}$ for which $\mathcal{W}_{x}^{N} \neq \emptyset$ and $\left|\mathcal{V}_{x}^{N}\right|=1$ holds, one after 
another, the construction described in Case (II). This yields a network $N^{\prime}$ such that $\mathcal{W}_{x}^{N^{\prime}}=\emptyset$ for all vertices $x$ in $N$ that satisfy Case (II). Hence, all such "Case (II)" vertices of $N$ satisfy Case (I) in $N^{\prime}$. Moreover, in each single modification step, the sets $\mathcal{W}_{a}^{N^{\prime}}$ have remained unchanged for all vertices $a \in \mathbb{S}$ that have not been considered thus far. Applying, one after another, the construction described in Case (III) to all $x \in \mathbb{S}$ for which $x \in \mathcal{W}_{x}^{N^{\prime}} \neq \emptyset$ and $\left|\mathcal{V}_{x}^{N^{\prime}}\right|>1$ holds results in the digraph $N^{*}$ as constructed in Definition 10. Arguing for all vertices $x$ that satisfy the conditions of Case (III) as in the proof for the "Case (II)" vertices of $N$, implies that $\mathcal{W}_{x}^{N^{*}}=\left\{w_{x}\right\}$ in $N^{*}$ and that $w_{x}$ is not contained in any multi-arc.

In summary, none of the applications of the constructions described in the proofs of Cases (II) and (III), respectively, introduces a multi-arc. Moreover, every $x \in \mathbb{S}$ satisfies $\mathcal{W}_{x}^{N^{*}}=\emptyset$ or $\mathcal{W}_{x}^{N^{*}}=\left\{w_{x}\right\}$ such that $w_{x}$ is not contained in any multi-arc. This, and the arguments preceding the discussion of Case (I) in the proof, imply that $N^{*}$ is a multi-arc free network.

Finally, Corollary 3 implies that the network $N$ can be constructed in polynomial-time. Moreover, it is easy to see that the sets $\mathcal{W}_{x}^{N}$ and $\mathcal{V}_{x}^{N}, x \in \mathbb{S}$, as well as the construction steps carried out in the Cases (II) and Case (III) to transform $N$ into $N^{*}$ can be performed in polynomial-time. Hence, $N^{*}$ can be obtained from $(T ; t, \sigma)$ in polynomial-time.

Now, let $(T ; t, \sigma)$ be an event-labeled gene tree, and let $N=(W, F)$ be the network associated to the simple subdivision $M^{\prime}$ of $M=M(T ; t, \sigma)$ as detailed in Definition 5. Let $N^{*}=\left(W^{*}, F^{*}\right)$ be the network without multi-arcs obtained from $N$ by the constructions detailed in Definition 7 and Proposition 3. As argued in the proof of Corollary 3, there is always a TreeNet-reconciliation map $\mu_{\kappa^{\prime}, f}$ from $(T ; t, \sigma)$ to $N$. The proof is, in particular, based on the fact that there is a folding map $f$ from $M^{\prime}$ to $N$. However, such a folding map may not exist for $M^{\prime}$ and $N^{*}$. Therefore, we will slightly adjust the map $\mu_{\kappa^{\prime}, f}$ to obtain a TreeNet-reconciliation map $\mu^{*}$ from $(T ; t, \sigma)$ to $N^{*}$.

To this end, we partition the vertex set of the original network $N=(W, F)$ as follows.

Definition 11. Let $N=(W, F)$ be a network on $\mathbb{S}$ and let $\mathbb{S}^{\prime}$ be the set of all $x \in \mathbb{S}$ with $\mathcal{W}_{x}^{N} \neq \emptyset$ holding. Put

$$
W_{1}=W \backslash W_{2} \text { and } W_{2}=\bigcup_{x \in \mathbb{S}^{\prime}}\left(\mathcal{W}_{x}^{N} \cup\{x\}\right)
$$

and

$$
F_{1}=\left\{a \in F \mid h_{N}(a), t_{N}(a) \in W_{1}\right\}
$$

Clearly, $W_{1}$ and $W_{2}$ form a partition of $W$. By Lemma 9(iii), there are no $\operatorname{arcs}(u, v) \in F$ with $u \in \mathcal{W}_{x}^{N}$ and $v \notin \mathcal{W}_{x}^{N}$ and $\mathcal{W}_{x}^{N} \cap \mathcal{W}_{y}^{N}=\emptyset$ for all distinct $x, y \in \mathbb{S}$. As an immediate consequence, we obtain the following

Observation 1. The subgraphs $N\left[W_{1}\right]$ of $N$ and $N^{*}\left[W_{1}\right]$ of $N^{*}$ induced by $W_{1}$ coincide, i.e., $N\left[W_{1}\right]=$ $N^{*}\left[W_{1}\right]$.

Definition 12. Let $(T=(V, E) ; t, \sigma)$ be an event-labeled gene tree on $\mathbb{G}$ and let $N=(W, F)$ be a network on $\mathbb{S}$ such that there is a TreeNet-reconciliation map $\mu$ from $(T=(V, E) ; t, \sigma)$ to $N$. Moreover, let $N^{*}=\left(W^{*}, F^{*}\right)$ be the multi-arc free network on $\mathbb{S}$ as in Definition 10 and let $W_{1}$ and $F_{1}$ be defined for $N$ as in Definition 11. The map $\mu^{*}: V \rightarrow W^{*} \cup F^{*}$ (w.r.t. $\mu$ ) is defined for all $v \in V$ as follows:

- If $v \in \mathbb{G}$, or $t(v)=\mathfrak{s}$, or $t(v)=\mathfrak{d}$ and $\mu(v) \in F_{1}$, then put $\mu^{*}(v):=\mu(v)$.

- Otherwise, there must exist a leaf $x \in \mathbb{S}$ such that either $h_{N}(\mu(v)) \in \mathcal{W}_{x}^{N}$ or $h_{N}(\mu(v))=x$, and we put $\mu^{*}(v):=(\operatorname{par}(x), x)$. 
Consider the event-labeled tree $(T ; t, \sigma)$ depicted in Fig. 6 and the network $N^{*}$ constructed from $(T ; t, \sigma)$ as specified in Proposition 4. The map $\mu^{*}$ is indicated in the bottom right network in Fig. 6. In what follows, we show that $\mu^{*}$ is well-defined and, in particular, a TreeNetreconciliation map from $(T ; t, \sigma)$ to $N^{*}$.

Proposition 4. Let $(T=(V, E) ; t, \sigma)$ be a well-behaved event-labeled gene tree on $\mathbb{G}$, let $N$ be the network on $\mathbb{S}$ associated to the simple subdivision of $(M(T ; t, \sigma), \chi)$ as in Definition 5 , and let $N^{*}$ be the multi-arc free network on $\mathbb{S}$ as in Definition 10.

Then, $\mu^{*}$ as in Def. 12 is a TreeNet-reconciliation map from $(T ; t, \sigma)$ to $N^{*}$.

Proof. In what follows, let $N=(W, F), N^{*}=\left(W^{*}, F^{*}\right)$ and put $M:=M(T ; t, \sigma)=(D, U)$. Moreover, let $W_{1}$ and $F_{1}$ be defined for $N$ as in Definition 11 .

Corollary 3 implies that there is always a TreeNet-reconciliation map $\mu:=\mu_{\kappa^{\prime}, f}$ from $(T ; t, \sigma)$ to $N$. In what follows, we show that $\mu^{*}$ (w.r.t. $\mu$ ) as in Definition 12 is a TreeNet-reconciliation map from $(T=(V, E) ; t, \sigma)$ to $N^{*}$.

Note, there may be arcs and vertices in $N$ that have been removed to obtain $N^{*}$ and new vertices and arcs may have been added in the construction of $N^{*}$. Hence, in order to show that $\mu^{*}$ is well-defined, we must ensure that either $\mu(v)$ is still contained in $N^{*}$ and that, otherwise, if we assign $\mu^{*}(v)=(\operatorname{par}(x), x)$ the condition $h_{N}(\mu(v)) \in \mathcal{W}_{x}^{N}$ or $h_{N}(\mu(v))=x$ is satisfied for this leaf $x \in \mathbb{S}$.

Clearly, if $v \in \mathbb{G}$, then $\mu^{*}(v)=\mu(v)=\sigma(v)$ is well-defined.

Now suppose that $v \in V^{0}$ with $t(v)=\mathfrak{s}$. We claim that $\mu(v) \in W_{1}$. To see this, note that $v$ must have two distinct children $v^{\prime}$ and $v^{\prime \prime}$ in $T$ for which $\sigma\left(L_{T}\left(v^{\prime}\right)\right) \neq \sigma\left(L_{T}\left(v^{\prime \prime}\right)\right)$ holds as $(T ; t, \sigma)$ is well-behaved. Thus, $\left|\sigma\left(L_{T}(v)\right)\right|>1$. By Properties (R1) and (R3) for $\mu$, we have $\mu(v) \succ_{N} x$ for all $x \in \sigma\left(L_{T}(v)\right)$. Hence, $\left|L_{N}(\mu(v))\right|>1$. Thus, $\mu(v) \in W$ is an inner vertex and there exists no $x \in \mathbb{S}$ such that $\mu(v) \in \mathcal{W}_{x}^{N}$. Thus, $\mu(v) \in W_{1}$, as claimed. Since none of the vertices in $W_{1}$ have been removed from $N$ to obtain $N^{*}, \mu(v)$ is still contained in $N^{*}$ for all speciation vertices $v$ of $(T ; t, \sigma)$ and we can put $\mu^{*}(v)=\mu(v) \in W^{*}$.

Now suppose that $v \in V^{0}$ with $t(v)=\mathfrak{d}$. If $\mu(v)=(u, w) \in F_{1}$, then $N\left[W_{1}\right]=N^{*}\left[W_{1}\right]$ implies that the $\operatorname{arc}(u, w)$ still exists in $N^{*}$ and we can put $\mu^{*}(v)=\mu(v) \in F^{*}$. If $\mu(v)=a=(u, w) \notin F_{1}$, then $u \notin W_{1}$ or $w \notin W_{1}$. If $u \notin W_{1}$, then $u \in \mathcal{W}_{x}^{N}$ for some $x \in \mathbb{S}$ and Lemma 9(ii) implies that either $w \in \mathcal{W}_{x}^{N}$ or $w=x$. In either case, $w$ is always contained in $W_{1}$ and hence, $w \in \mathcal{W}_{x}^{N}$ or $w=x$ (and thus, $a=(\operatorname{par}(x), x))$ for some $x \in \mathbb{S}$. In this case, we put $\mu^{*}(v)=(\operatorname{par}(x), x) \in F^{*}$.

In summary, it follows that $\mu^{*}$ is well-defined,

To see that $\mu^{*}$ is a reconciliation map from $(T ; t, \sigma)$ to $N^{*}$, we need to show that Properties (R1) - (R3) hold. Since Property (R1) clearly holds as $\mu(v)=\sigma(v)$ for all $v \in \mathbb{G}$, it suffices to restrict attention to Properties (R2) and (R3). In what follows, let $V_{\mu} \subseteq V$ be the set of vertices $v \in V$ such that $\mu^{*}(v)=\mu(v)$.

We start with establishing Property (R3). We first recap, that the ancestor relationship between the vertices in $W_{1} \cup \mathbb{S}$ has not been changed in $N^{*}$, that is, $w, w^{\prime} \in W_{1} \cup \mathbb{S}$ with $w \prec_{N} w^{\prime}$ (resp. $\left.w=w^{\prime}\right)$ in $N$ implies $w \prec_{N^{*}} w^{\prime}$ (resp. $\left.w=w^{\prime}\right)$ in $N^{*}$. The latter also implies that the relative order of the $\operatorname{arcs}(a, b) \in F_{1}$ under $\prec_{N}$ has not been changed in $N^{*}$.

To see Property (R3), suppose that $u, v \in V$ with $v \prec_{T} u$.

To see Property (R3.i), assume that $t(v)=t(u)=\mathfrak{d}$. We need to show that $\mu^{*}(v) \preceq_{N^{*}} \mu^{*}(u)$. Clearly, if $u, v \in V_{\mu}$ then $\mu^{*}(v)=\mu(v) \preceq_{N} \mu(u)=\mu^{*}(u)$ as $\mu$ satisfies Property (R3.i). Since the relative order of the arcs in $F_{1}$ has not been changed by the construction of $N^{*}$ it follows that $\mu^{*}(v) \preceq_{N^{*}} \mu^{*}(u)$. Hence, Property (R3.i) holds in this case.

So assume that $u, v \notin V_{\mu}$. Hence, $\mu(u)=a \in F \backslash F_{1}$ and, as argued above, $h_{N}(a) \in \mathcal{W}_{x}^{N}$ or $a=(\operatorname{par}(x), x)$ for some $x \in \mathbb{S}$. Note, $\mu(v) \preceq_{N} \mu(u)=a$ as $\mu$ satisfies Property (R3). This combined with Lemma 9, implies that there cannot be an arc $(w, z)$ in $N$ with $w \in \mathcal{W}_{x}^{N}$ and $z \notin \mathcal{W}_{x}^{N}$ or $z \neq x$. Thus, for $a^{\prime}=\mu(v)$ we have $t_{N}\left(a^{\prime}\right) \in \mathcal{W}_{x}^{N}$ and therefore, $h_{N}\left(a^{\prime}\right) \in \mathcal{W}_{x}^{N}$ or $h_{N}\left(a^{\prime}\right)=x$. By 
construction of $\mu^{*}$ we have, therefore, $\mu^{*}(u)=\mu^{*}(v)=(\operatorname{par}(x), x)$. Hence, Property (R3.i) holds in this case.

Assume next that one of $u$ and $v$ is contained in $V_{\mu}$ whereas the other is not. Note that if $u \notin V_{\mu}$, then $\mu(u) \in F \backslash F_{1}$. Similar arguments as in the latter case imply $\mu(v) \in F \backslash F_{1}$. Hence, $v \notin V_{\mu}$. Therefore, $u \in V_{\mu}$ and $v \notin V_{\mu}$ must hold. Thus, $\mu(u)=\mu^{*}(u) \in F_{1} \subseteq F^{*}$ and $h_{N}(\mu(v)) \in \mathcal{W}_{x}^{N}$ or $\mu(v)=(\operatorname{par}(x), x)$ for some $x \in \mathbb{S}$. Hence, $\mu^{*}(v)=(\operatorname{par}(x), x)$. Since $\mu$ satisfies Property (R3.i) in $N$ we have $x \prec_{N} \mu(v) \preceq_{N} \mu(u)=\mu^{*}(u)$ and, therefore, that $x \prec_{N^{*}} \mu^{*}(v) \preceq_{N^{*}} \mu^{*}(u)$. In combination, we obtain that Property (R3.i) is satisfied for all duplication vertices of $N$.

To see that Property (R3.ii) also holds, assume that at least one of $t(u)=\mathfrak{s}$ and $t(v)=\mathfrak{s}$ holds. If $t(v)=\mathfrak{s}$ then $v \in V_{\mu}$. Again, this implies that $u \in V_{\mu}$, since $v \prec_{T} u, \mu$ satisfies Property (R3.ii) and Lemma 9(ii) holds. Moreover, Thus, $\mu^{*}(v)=\mu(v) \prec_{N} \mu(u)=\mu^{*}(u)$. Since $u, v \in V_{\mu}$ and the ancestor relationships in $N$ are preserved in $N^{*}$ for all vertices in $W_{1} \cup \mathbb{S}$, it follows that $\mu^{*}(v) \prec_{N^{*}}$ $\mu^{*}(u)$.

So assume that $t(u)=\mathfrak{s}$. Then $u \in V_{\mu}$. Note that we may assume w.l.o.g. that $t(v)=\mathfrak{d}$ as otherwise $\mu^{*}(v)=\mu(v)$ and $\mu^{*}(u)=\mu(u)$ must hold. Thus, similar arguments as before imply that $\mu^{*}(v) \prec_{N^{*}} \mu^{*}(v)$. Note also that we may assume that $\mu(v) \notin F_{1}$ as otherwise we have again that $\mu^{*}(v)=\mu(v)$ and $\mu^{*}(u)=\mu(u)$ which, in turn, implies $\mu^{*}(v) \prec_{N^{*}} \mu^{*}(v)$. Then $h_{N}(\mu(v)) \in$ $\mathcal{W}_{x}^{N}$ or there exists some $x \in \mathbb{S}$ such that $\mu(v)=(\operatorname{par}(x), x)$. Hence, $\mu^{*}(v)=(\operatorname{par}(x), x)$. Thus, $x \prec_{N} \mu(v) \prec_{N} \mu(u)=\mu^{*}(u)$. Since the ancestor relationships in $N$ are preserved in $N^{*}$ for all vertices in $W_{1} \cup \mathbb{S}$ and since $\mu^{*}(v)=(\operatorname{par}(x), x)$ is the lowest possible choice for $\mu^{*}(v)$, it follows that $x \prec_{N^{*}} \mu^{*}(v) \prec_{N^{*}} \mu^{*}(u)$. This concludes the proof of Property (R3.ii) and, thus the proof of Property (R3).

It remains to show that Property (R2) holds. Clearly, by construction of $\mu^{*}$, Property (R2.ii) is satisfied in $N^{*}$.

To see Property (R2.i), assume that $v \in V$ with $t(v)=\mathfrak{s}$ and children $v_{1}, \ldots, v_{k} \in V, k \geq 2$. We need to show that $\mu^{*}(v) \in Q_{N^{*}}^{2}\left(\mu^{*}\left(v_{1}\right), \ldots, \mu^{*}\left(v_{k}\right)\right)$. Since $\mu$ is a TreeNet-reconciliation map from $(T ; t, \sigma)$ to $N$, we clearly have $\mu(v) \in Q_{N}^{2}\left(\mu\left(v_{1}\right), \ldots, \mu\left(v_{k}\right)\right)$. Thus, there exist $i, j \in\{1, \ldots, k\}$ such that $\mu\left(v_{i}\right)$ and $\mu\left(v_{j}\right)$ (note that $\mu\left(v_{i}\right)=\mu\left(v_{j}\right)$ might be possible) are separated by $\mu(v)$ in $N$. Therefore, there exists a vertex $w^{\prime} \in W$ such that $\left(\mu(v), w^{\prime}\right)$ is the first arc on a directed path from $\mu(v)$ to $\mu\left(v_{i}\right)$ in $N$. Similarly, there exists a vertex $w^{\prime \prime} \in W$ such that $\left(\mu(v), w^{\prime \prime}\right)$ is the first arc on a directed path from $\mu(v)$ to $\mu\left(v_{j}\right)$ in $N$. Note that $w^{\prime}=w^{\prime \prime}$ or $w^{\prime} \neq w^{\prime \prime}$ might hold. In the first case, we might have multi-arcs between $\mu(v)$ and $w^{\prime}$ in $N$.

Assume first that $w^{\prime} \neq w^{\prime \prime}$. We distinguish between the cases that $w^{\prime}, w^{\prime \prime} \in W_{1}$ (Case (C1)) and that at least one of $w^{\prime}$ and $w^{\prime \prime}$ is not contained in $W_{1}$ (Case (C2)).

Case (C1): Since $w^{\prime}, w^{\prime \prime} \in W_{1}$, we have for all $x \in \mathbb{S}$ that $w^{\prime}, w^{\prime \prime} \notin \mathcal{W}_{x}^{N}$. By construction of $N^{*}$, both vertices $w^{\prime}$ and $w^{\prime \prime}$ are also contained in $N^{*}$. By the definition of $\mu^{*}$, we have that $\mu^{*}\left(v_{i}\right)=\mu\left(v_{i}\right)$ in case $v \in \mathbb{G}$, or $t(v)=\mathfrak{s}$, or $t(v)=\mathfrak{d}$ and $\mu\left(v_{i}\right) \in F_{1}$, and that, otherwise, there exists some $x \in \mathbb{S}$ such that $\mu^{*}\left(v_{i}\right)=(\operatorname{par}(x), x)$. Since the ancestor relationship of all vertices in $W_{1} \cup \mathbb{S}$ is preserved in $N^{*}$ it follows that $\left(\mu(v), w^{\prime}\right) \succeq_{N^{*}} \mu^{*}\left(v_{i}\right)$. Put differently, there exists a directed path from $\mu^{*}(v)=\mu(v)$ to $\mu^{*}\left(v_{i}\right)$ in $N^{*}$ that contains the arc $\left(\mu^{*}(v), w^{\prime}\right)$. Note that $\mu^{*}\left(v_{i}\right)=$ $\mu\left(v_{i}\right)=\left(\mu^{*}(v), w^{\prime}\right)$ might hold. Similarly, there exists a directed path from $\mu^{*}(v)$ to $\mu^{*}\left(v_{i}\right)$ in $N^{*}$ that contains the $\operatorname{arc}\left(\mu^{*}(v), w^{\prime \prime}\right)$. Hence, $\mu^{*}(v)$ separates $\mu^{*}\left(v_{i}\right)$ and $\mu^{*}\left(v_{j}\right)$ in $N^{*}$. Therefore, $\mu^{*}(v) \in Q_{N^{*}}^{2}\left(\mu^{*}\left(v_{1}\right), \ldots, \mu^{*}\left(v_{k}\right)\right)$.

Case (C2): W.1.o.g. assume that $w^{\prime} \notin W_{1}$. Thus, either $w^{\prime} \in \mathcal{W}_{x}^{N}$ or $\mathcal{W}_{x}^{N} \neq \emptyset$ and $w^{\prime}=x$, for some $x \in \mathbb{S}$. We claim that, in $N^{*}$, either $\operatorname{par}(x)=\mu(v)$ or $\operatorname{par}(x)=w_{x}$ holds, where $w_{x}$ is the unique vertex added by replacing $\mathcal{W}_{x}^{N}$ as in Definition 10. To see this, note first that since all vertices in $\mathcal{W}_{x}^{N}$ and their incident arcs were removed from $N$ and either the arc $(\mu(v), x)$ or the two $\operatorname{arcs}\left(\mu(v), w_{x}\right)$ and $\left(w_{x}, x\right)$ were added to obtain $N^{*}$ it follows that either $\operatorname{par}(x)=\mu(v)$ or $\operatorname{par}(x)=w_{x}$, as claimed. Furthermore, since there is a directed path from $\mu(v)$ to $\mu\left(v_{i}\right)$ in $N$ with $\operatorname{arc}\left(\mu(v), w^{\prime}\right)$, we have $\mu\left(v_{i}\right)=\left(\mu(v), w^{\prime}\right)$ or $w^{\prime} \succeq_{N} \mu\left(v_{i}\right)$. Hence, $\mu\left(v_{i}\right) \in \mathcal{W}_{x}^{N}$ or $\mu\left(v_{i}\right)=x$. 
Note that $\mu\left(v_{i}\right) \in \mathcal{W}_{x}^{N}$ or $\mu\left(v_{i}\right)=x$ implies in particular that, in $N^{*}$, we have $\mu^{*}\left(v_{i}\right)=(\operatorname{par}(x), x)$ or $\mu^{*}\left(v_{i}\right)=x$.

We next claim that there exists some $1 \leq \ell \leq k$ distinct from $i$ such that $\mu^{*}\left(v_{\ell}\right) \neq(\operatorname{par}(x), x)$. To see this claim note first that since $\mu$ satisfies Property (R3) and $\mu(u)=\sigma(u) \in \mathbb{S}$ for all $u \in \mathbb{G}$, it follows that $\mu\left(v_{i}\right) \succeq_{N} \sigma(u)$ for all $u \in \mathbb{G}$ with $v_{i} \succeq_{T} u$. Thus, if $\mu\left(v_{i}\right) \in \mathcal{W}_{x}^{N}$ then $\sigma\left(L_{T}\left(v_{i}\right)\right)=\{x\}$. Since $\sigma\left(L_{T}\left(v_{i}\right)\right)=\{x\}$ also holds in case of $\mu\left(v_{i}\right)=x$ our assumption that $T$ is well-behaved implies that there is some $1 \leq \ell \leq k$ distinct from $i$ such that $\sigma\left(L_{T}\left(v_{\ell}\right)\right) \neq \sigma\left(L_{T}\left(v_{i}\right)\right)=\{x\}$. Let $y \in \sigma\left(L_{T}\left(v_{\ell}\right)\right)$ with $y \neq x$. Since $v_{\ell} \succeq_{T} u$ for some $u \in \mathbb{G}$ with $\mu^{*}(u)=\sigma(u)=y$ and $\mu^{*}$ satisfies Property (R3) in $N^{*}$, we have $\mu^{*}\left(v_{\ell}\right) \succeq_{N^{*}} y$. Therefore, $\mu\left(v_{\ell}\right)$ cannot be contained in $\mathcal{W}_{x}^{N}$. Hence, $\mu^{*}\left(v_{\ell}\right) \neq(\operatorname{par}(x), x)$, as claimed.

As shown above, $\mu^{*}\left(v_{i}\right)=(\operatorname{par}(x), x)$ or $\mu^{*}\left(v_{i}\right)=x$. In either case, by construction of $N^{*}$, the vertex $\operatorname{par}(x)$ has only one child, namely the vertex $x$. Since $x \neq y$, it follows that if $\mu^{*}\left(v_{\ell}\right) \succeq_{N^{*}} y$ then $\operatorname{par}(x) \succeq_{N^{*}} \mu^{*}\left(v_{\ell}\right)$. The latter combined with $\mu^{*}(v) \succ_{N^{*}} \mu^{*}\left(v_{\ell}\right)$ implies that there is an alternative directed path from $\mu^{*}(v)$ to $\mu^{*}\left(v_{\ell}\right)$ that has only $\mu^{*}(v)$ in common with the directed path from $\mu^{*}(v)$ to $\mu^{*}\left(v_{i}\right)$. Thus, $\mu^{*}(v)$ separates $\mu^{*}\left(v_{i}\right)$ and $\mu^{*}\left(v_{\ell}\right)$ and $\mu^{*}(v) \in Q_{N^{*}}^{2}\left(\mu^{*}\left(v_{1}\right), \ldots, \mu^{*}\left(v_{k}\right)\right)$.

To finish the proof that $\mu^{*}$ satisfies Property (R2.i) it remains to consider the case that $w^{\prime}=w^{\prime \prime}$. Then $\left(\mu(v), w^{\prime}\right)$ and $\left(\mu(v), w^{\prime \prime}\right)$ are parallel arcs. By construction of $N^{*}$, if there are parallel arcs between two vertices $\mu(v)$ and $w^{\prime}$ in $N$, then $w^{\prime}=\operatorname{par}(x) \in \mathcal{W}_{x}^{N}$ must hold for some $x \in \mathbb{S}$. Hence, $w^{\prime} \in \mathcal{W}_{x}^{N}$. Similar arguments as in the proof of Case (C2) imply that $\mu^{*}(v) \in Q_{N^{*}}^{2}\left(\mu^{*}\left(v_{1}\right), \ldots, \mu^{*}\left(v_{k}\right)\right)$.

In summary, $\mu^{*}$ satisfies Property (R2). This completes the proof that $\mu^{*}$ is a TreeNetreconciliation map from $(T ; t, \sigma)$ to $N^{*}$.

As suggested already by Fig.6, the network $N^{*}$ obtained from $N$ as described in Definition 10 displays all triples in the event-labeled gene tree in Fig. 6. That this is not a coincidence is the purpose of the next result.

Proposition 5. Let $(T ; t, \sigma)$ be an event-labeled gene tree on $\mathbb{G}$ and let $N$ be a species network on $\mathbb{S}$ obtained from the simple subdivision of the MUL-tree $(M(T ; t, \sigma), \chi)$ as in Definition 5. Then the multi-arc free network $N^{*}$ on $\mathbb{S}$ displays all triples in $\mathcal{S}(T ; t, \sigma)$.

Proof. Put $M:=M(T ; t, \sigma)$ and let $N=(W, F)$ and $N^{*}=\left(W^{*}, F^{*}\right)$.

Suppose that $A, B, C \in \mathbb{S}$ such that $A B \mid C \in \mathcal{S}(T ; t, \sigma)$. Then there are three elements $a, b, c \in \mathbb{G}$ with pairwise distinct $\sigma(a)=A, \sigma(b)=B$ and $\sigma(c)=C$ such that the (undirected) path between $a$ and $b$ in $T$ does not intersect the directed path from $\rho_{T}$ to $c$ in $T$. In particular, putting $v:=$ $\operatorname{lca}_{T}(a, b, c)$ we have $t(v)=\mathfrak{s}$. Thus, $u:=\operatorname{lca}(a, b) \prec_{T} v$. In what follows, we write $v^{W}$ for every $v \in V$ that is still contained in $W$. Moreover, we say that two paths $P\left(v_{1}, v_{k}\right)$ with $k \geq 1$ and $P\left(w_{1}, w_{\ell}\right)$ with $\ell \geq 1$ in a graph are internal vertex disjoint, if $P\left(v_{1}, v_{k}\right)$ and $P\left(w_{1}, w_{\ell}\right)$ share at most one vertex from the set $\left\{v_{1}, v_{k}, w_{1}, w_{\ell}\right\}$.

Observe that any directed path $P=\left(v_{1}, \ldots, v_{k}\right)$ in $T$ with $k \geq 1$, for which the corresponding vertices $v_{1}^{W}, \ldots, v_{k}^{W}$ are contained in $W_{1}$, also forms a directed path $P_{W}:=\left(v_{1}^{W}, \ldots, v_{k}^{W}\right)$ in $N$ and thus, in $N\left[W_{1}\right]$. Since $N\left[W_{1}\right]$ and $N^{*}\left[W_{1}\right]$ coincide, it follows that $P_{W}$ is contained in $N^{*}$. In particular, if there are internal vertex disjoint directed paths $P=\left(v_{1}, \ldots, v_{k}\right)$ with $k \geq 1$ and $P^{\prime}=$ $\left(w_{1}, \ldots, w_{\ell}\right)$ with $\ell \geq 1$ in $T$, then the directed paths $P_{W}=\left(v_{1}^{W}, \ldots, v_{k}^{W}\right)$ and $P_{W}^{\prime}=\left(w_{1}^{W}, \ldots, w_{\ell}^{W}\right)$ exist in $N$. Moreover, $P_{W}$ and $P_{W}^{\prime}$ are internal vertex disjoint, whenever $v_{k}^{W}, w_{\ell}^{W} \in W_{1}$, as this implies, $v_{1}^{W}, \ldots, v_{k-1}^{W}, w_{1}^{W}, \ldots, w_{\ell-1}^{W} \in W_{1}$ as well.

Since $\left|\sigma\left(L_{T}(v)\right)\right|>1$ and $\left|\sigma\left(L_{T}(u)\right)\right|>1$, we have by construction of $N$ that $\left|L_{N}\left(v^{W}\right)\right|>1$ and $\left|L_{N}\left(u^{w}\right)\right|>1$. Thus, $v^{W}, u^{W} \in W_{1}$. Let $P_{1}$ be the unique path from $v$ to $u$ in $T$. Since $v^{W}, u^{W} \in W_{1}$, for any vertex $w$ of $P_{1}$, we have $w^{W} \in W_{1}$. Thus, the (directed) path $P_{1}^{*}$ from $v^{W}$ to $u^{W}$ exists in $N$ and hence, also in $N^{*}$.

Consider now the unique path $P_{2}=\left(u, u_{1}, \ldots u_{k}, a\right)$ in $T$. For $\sigma(a)=A$, we distinguish between the cases that $|\chi(A)|=1$ and $|\chi(A)|>1$. 
If $|\chi(A)|=1$, then the arc $e=\left(u_{k}, A\right)$ is contained in the simple subdivision $M^{\prime}$ of $M$ and has not been identified with any other arc as part of the construction of $N$. Since $M$ is a MUL-tree and $|\chi(A)|=1$, it is easy to see that $u_{k} \in W_{1}$. It follows that $P_{2}^{\prime}=\left(u^{W}, u_{1}^{W}, \ldots u_{k}^{W}, A\right)$ is entirely contained in $N\left[W_{1}\right]$, and thus, in $N^{*}\left[W_{1}\right]$.

So assume that $|\chi(A)|>1$. Then $e=\left(u_{k}, A\right)$ in $M$ was replaced by the two $\operatorname{arcs}\left(u_{k}, v_{e}\right)$ and $\left(v_{e}, A\right)$ as part of the construction of $M^{\prime}$. Moreover, the arc $\left(v_{e}, A\right)$ has been identified with other arcs that have head in $\chi(A)$ to obtain the unique $\operatorname{arc}(\operatorname{par}(A), A)$ in $N$. Thus, the path $P_{2}^{\prime \prime}=\left(u_{0}^{W}:=\right.$ $\left.u^{W}, u_{1}^{W}, \ldots u_{k}^{W}, v_{e}, A\right)$ exists in $N$. Let $u_{i}^{W}$ be the first vertex on $P_{2}^{\prime \prime}$ that is contained in $\mathcal{W}_{A}^{N}$. Note, $u^{W} \in W_{1}$ implies $u^{W} \neq u_{i}^{W}$. For the construction of $N^{*}$ from $N$, the subpath $\left(u_{i-1}^{W}, u_{i}^{W}, \ldots, u_{k}^{W}, v_{e}, A\right)$ of $P_{2}^{\prime \prime}$ has been replaced by either the $\operatorname{arc}\left(u_{i-1}^{W}, A\right)$ or by the two $\operatorname{arcs}\left(u_{i-1}^{W}, w_{A}\right)$ and $\left(w_{A}, A\right)$. Thus, the last arc on the directed path $P_{2}^{\prime \prime \prime}=\left(u^{W}, u_{1}^{W}, \ldots u_{i-1}^{W}, \ldots, A\right)$ in $N^{*}$ is either $\left(u_{i-1}^{W}, A\right)$ or $\left(w_{A}, A\right)$. Let us denote by $P_{2}^{*}$ the respective path $P_{2}^{\prime}$ or $P_{2}^{\prime \prime \prime}$ from $u^{W}$ to $A$ in $N^{*}$.

Analogously to $P_{2}$, by repeating the latter arguments for the unique paths $P_{3}=(u, \ldots, b)$, resp., $P_{4}=(v, \ldots, c)$ in $T$, we can construct the paths $P_{3}^{*}$ from $u^{W}$ to $B$ in $N^{*}$, resp., $P_{4}^{*}$ from $v^{W}$ to $C$ in $N^{*}$.

Since all path $P_{1}, P_{2}, P_{3}$ and $P_{4}$ are pairwise internal vertex disjoint and since $A, B, C$ are pairwise distinct, the construction of the respective paths $P_{i}^{*}, 1 \leq i \leq 4$ implies that $P_{1}^{*}, P_{2}^{*}, P_{3}^{*}$ and $P_{4}^{*}$ are pairwise internal vertex disjoint. In particular, $P_{1}^{*}$ shares with $P_{2}^{*}$, resp., $P_{3}^{*}$ only vertex $u^{W}$, $P_{1}^{*}$ and $P_{4}^{*}$ share only vertex $v^{W}, P_{2}^{*}$ and $P_{3}^{*}$ share only vertex $u^{W}$, and $P_{4}^{*}$ does not share any vertex with $P_{2}^{*}$ and $P_{3}^{*}$. In summary, after the suppression of vertices with in- and outdegree one in the minimal subgraph of $N^{*}$ that contains the paths $P_{1}^{*}, \ldots, P_{4}^{*}$, we obtain a binary reduced tree $T^{\prime}$ on $\{A, B, C\}$ such that the (undirected) path between $A$ and $B$ does not intersect the path from $\rho_{T^{\prime}}$ to $C$. Therefore, $A B \mid C$ is displayed in $N^{*}$.

Taken Propositions 3, 4 and 5 together we obtain

Theorem 6. Suppose $(T ; t, \sigma)$ is a well-behaved event-labeled gene tree. Then there is a multiarc free network $N^{*}$ for $(T ; t, \sigma)$ that can be constructed in polynomial time and that displays all triples in $\mathcal{S}(T ; t, \sigma)$.

The next result is well-known [32], but using the last result we can give a simple alternative proof.

Corollary 4. For any set $R$ of triples there is a multi-arc free network that displays each triple in $R$.

Proof. If $R=\emptyset$, the statement is trivially satisfied. Hence, let $R=\left\{r_{1}, \ldots, r_{m}\right\}, m \geq 1$, be a non-empty set of triples. Put $L_{R}:=\cup_{r \in R} L(r)$.

We construct an event-labeled gene tree $(T ; t, \sigma)$ as follows: To each triple $r_{i}=x_{i 1} x_{i 2} \mid x_{i 3} \in R$, $1 \leq i \leq m$, we associate a triple $T_{i}=a_{i 1} a_{i 2} \mid a_{i 3}$ with root $\rho_{i}$ making sure that $L\left(T_{i}\right) \cap L\left(T_{j}\right)=\emptyset$ for all $1 \leq i<j \leq m$. We start with the empty graph $T=\emptyset$ and add first all triples $T_{1}, \ldots T_{m}$ to $T$. Next, we add a single new vertex $\rho_{T}$ to $T$ and an $\operatorname{arc}\left(\rho_{T}, \rho_{i}\right), 1 \leq i \leq m$. By construction, $T$ is a reduced phylogenetic tree. Note that although $x_{i r}=x_{j s}$ might hold, we always have $a_{i r} \neq a_{j s}$, $1 \leq i<j \leq m$ and $r, s \in\{1,2,3\}$. Hence, $L(T)=\left\{a_{11}, a_{12}, a_{13}, \ldots, a_{k 1}, a_{k 2}, a_{k 3}\right\}$. Finally, we put $t(v)=\mathfrak{s}$ for all inner vertices $v \neq \rho_{T}$ and $t\left(\rho_{T}\right)=\mathfrak{d}$ and define the map $\sigma: L(T) \rightarrow L_{R}$ by putting $\sigma\left(a_{i j}\right)=x_{i j}, 1 \leq i \leq m$ and $1 \leq j \leq 3$.

By construction, $(T ; t, \sigma)$ is an event-labeled gene tree and each triple in $(T ; t, \sigma)$ that is rooted at a speciation vertex corresponds to one of the triples $T_{i}, 1 \leq i \leq m$. By the choice of $\sigma$ we immediately obtain $\mathcal{S}(T ; t, \sigma)=R$.

Next, we construct the network $N$ obtained from a simple subdivision of the MUL-tree $(M(T ; t, \sigma), \chi)$ as described in Definition 5. Finally, we construct $N^{*}$ as in Definition 10 and apply Proposition 3 to conclude that $N^{*}$ is a multi-arc free network. Moreover, Proposition 5 implies that $N^{*}$ displays all triples in $R$, which completes the proof. 


\section{The Relationship Between Reconciliations for MUL- Trees and Networks}

In this section we show that, given an event-labeled gene tree $(T ; t, \sigma)$, and a network $N$, then $N$ is a species network for $(T ; t, \sigma)$ if and only if $U^{*}(N)$ is a pseudo-MUL-tree for $(T ; t, \sigma)$ (see Theorem 7). Note that the "if" direction of this result follows from Theorem 5.

In what follows, let $(T=(V, E) ; t, \sigma)$ be an event-labeled gene tree, let $N=(W, F)$ be a multiarc free species network on $\mathbb{S}$, and let $\mu$ be a reconciliation map between from $(T ; t, \sigma)$ to $N$. Moreover, let $f=\left(f_{V}, f_{E}\right)$ denote a folding map from a pseudo MUL-tree $(M, \chi)=((D, U), \chi)$ labeled by $\mathbb{S}$ to $N$. By Proposition 2, we may assume w.l.o.g. that $(M, \chi)=\left(U^{*}(N), \chi^{*}\right)$ and that $\rho_{N}=\rho_{M}$.

In the following, we first define a map $\kappa_{\mu, f}: V \rightarrow\left(D \backslash D^{1}\right) \cup U$ associated to $\mu$ and $f$, and we then show that $\kappa_{\mu, f}$ is a MUL-reconciliation from $(T ; t, \sigma)$ to $(M, \chi)$ (Proposition 6), which will immediately imply Theorem 7. We shall define the map $\kappa_{\mu, f}: V \rightarrow\left(D \backslash D^{1}\right) \cup U$ in a top-down fashion. Although the definition is quite long and technical, it follows in a quite straight-forward fashion from the lifting properties of a folding map (see Fig. 7 for an illustrative example).

In order to define $\kappa_{\mu, f}$ we begin with an observation. Let $x, y \in W$ be two distinct vertices with $y \prec_{N} x$ and assume that $P(x, y)=\left(w_{0}=x, w_{1}, \ldots, w_{k}, w_{k+1}=y\right), k \geq 0$ is a directed path in $N$ from $x$ to $y$. For all $0 \leq i \leq k$, let $a_{i}$ denote the arc $\left(w_{i}, w_{i+1}\right)$ between $w_{i}$ and $w_{i+1}$ in $N$. Note, that $a_{i}$ is well-defined, since $N$ is multi-arc free and thus, there is at most one arc between any two vertices of $N$. Observe that since $f$ is a folding from $(M, \chi)=\left(U^{*}(N), \chi^{*}\right)$ into $N$ there exists for all $0 \leq i \leq k$ a unique $\operatorname{arc} \widetilde{a_{i}^{w_{i}}} \in U$ obtained by lifting the arc $a_{i}$ of $N$ at the vertex $w_{i}$ of $N$.

Now, suppose $v \in V$. To define $\kappa_{\mu, f}(v)$ in a top-down fashion, we begin with the base case $v=\rho_{T}$. Since we assume that $|L(T)| \geq 2$, either (i) $t(v)=\mathfrak{s}$ or (ii) $t(v)=\mathfrak{d}$.

Case (i): If $t(v)=\mathfrak{s}$, then $\mu(v)$ is a vertex of $N$. Note that $\mu(v) \neq \rho_{N}$ must hold as $\mu$ satisfies Property (R2.i). Hence, the directed path $P\left(\rho_{N}, \mu(v)\right)=\left(w_{0}=\rho_{N}, w_{1}, \ldots, w_{k}, w_{k+1}=\mu(v)\right)$ must cross at least one arc of $N$, i.e, $k \geq 0$. Since $f$ is a folding of $M$ into $N$ there exists a path in $M$ from $\rho_{M}=\rho_{N}$ to the head $h_{M}\left(\widetilde{a_{k}^{w_{k}}}\right)$ of the arc $\widetilde{a_{k}^{w_{k}}} \in U$ obtained by lifting $a_{k}$ at $w_{k}=\operatorname{par}(\mu(v))$. Then we put $\kappa_{\mu, f}(v)=h_{M}\left(\widetilde{a_{k}^{w_{k}}}\right)$.

Case (ii): If $t(v)=\mathfrak{d}$ then $\mu(v)$ is an arc of $N$. If $\rho_{N}=t_{N}(\mu(v))$ then we put $\kappa_{\mu, f}(v)=\widetilde{a_{0}^{w_{0}}}$. In case $\rho_{N} \neq t_{N}(\mu(v))$, the directed path $P\left(\rho_{N}, t_{N}(\mu(v))\right)=\left(w_{0}=\rho_{N}, w_{1}, \ldots, w_{k}, w_{k+1}=\mu(v)\right)$ has at least one arc. Since $f$ is a folding from $M$ to $N$ there exists a path from $\rho_{M}$ to the tail $t_{M}\left(\widetilde{a_{k}^{w_{k}}}\right)$ of the arc $\widetilde{a_{k}^{w_{k}}} \in U$ obtained by lifting $a_{k}$ at $w_{k}$. So we put $\kappa_{\mu, f}(v)=\widetilde{a_{k}^{w_{k}}}$.

Now, assume that $v \neq \rho_{T}$ is such that $\kappa_{\mu, f}(u)$ has already been defined for all vertices $u$ of $T$ that are above $v$. Let $w \in V$ denote the parent of $v$ which must exist as $v \neq \rho_{T}$. If $\mu(v)=\mu(w)$ then we put $\kappa_{\mu, f}(v)=\kappa_{\mu, f}(w)$. So assume that $\mu(v) \neq \mu(w)$. Note that $\mu(w) \neq \rho_{N}$, independent of whether $t(w)=\mathfrak{s}$ or $t(w)=\mathfrak{d}$. We distinguish between the two cases that $v$ is a leaf of $T$ and that it is not.

If $v$ is a leaf of $T$ then $\mu(v)$ must be a leaf of $N$. Consider the directed path $P$ obtained by extending the directed path $P\left(\rho_{N}, \mu(w)\right)$ of $N$ by the directed path $P\left(h_{N}(\mu(w)), \mu(v)\right)$. Note that $P$ must contain at least two arcs. Then we put $\kappa_{\mu, f}(v)=h_{M}\left(\widetilde{b_{q}^{u_{q}}}\right)$ where $u_{q}$ is the last but one vertex on $P$ and $b_{q}$ is the $\operatorname{arc}\left(u_{q}, \mu(v)\right)$ of $N$.

So assume that $v$ is not a leaf of $T$. To define $\kappa_{\mu, f}$, we need to distinguish between the cases that (a) $t(v)=t(w)=\mathfrak{s}$, (b) $t(v)=\mathfrak{s}$ and $t(w)=\mathfrak{d}$, (c) $t(v)=\mathfrak{d}$ and $t(w)=\mathfrak{s}$, and (d) $t(v)=t(w)=\mathfrak{d}$ (note that since $\mu$ is a TreeNet-reconciliation from $(T ; t, \sigma)$ to $N$, Property (R3) implies that $\mu(v) \preceq_{N} \mu(w)$ holds in all of these cases).

Case (a): If $t(v)=t(w)=\mathfrak{s}$, then $\mu(v)$ and $\mu(w)$ are vertices of $N$. Assume first that none of the children of $w$ have obtained an image under $\kappa_{\mu, f}$. Consider the directed path $P$ obtained by 


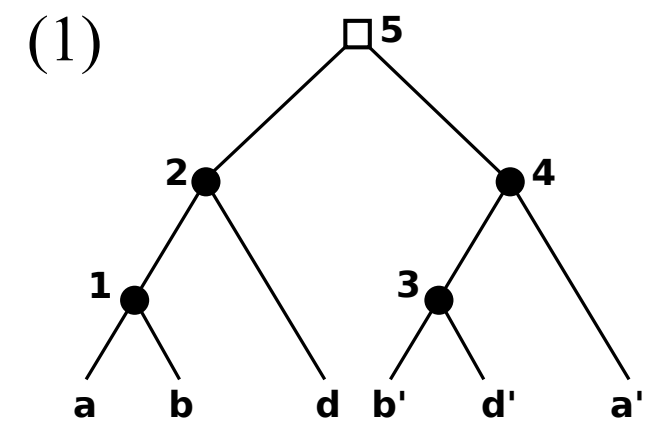

(3)

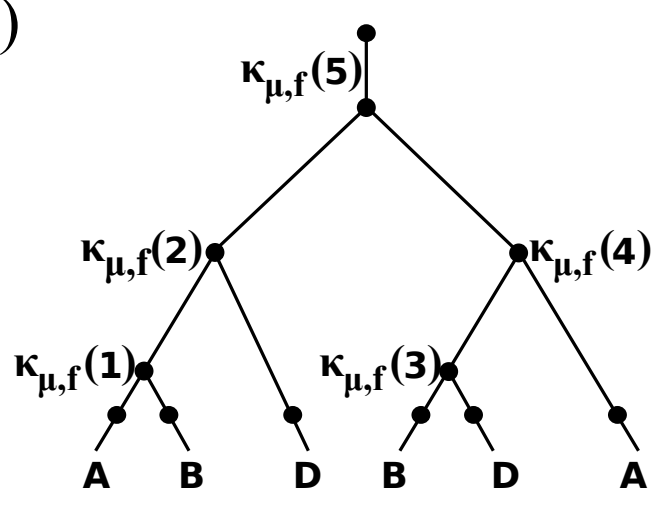

(2)

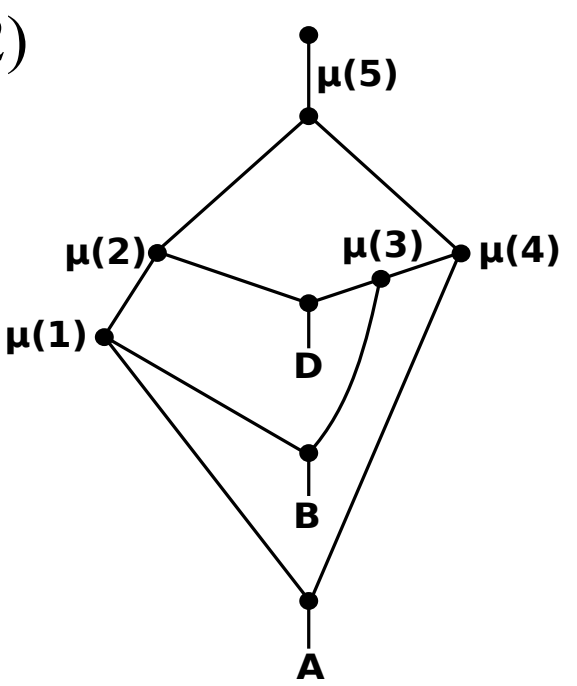

Figure 7

Panel (1) shows the event-labeled gene tree $(T ; t, \sigma)$ from Fig. 5 ignoring the labels of the inner vertices for the moment. Speciation and duplication events are represented as $\bullet$ and $\square$, respectively. Ignoring the labels of the inner vertices also in the remaining two panels for the moment, we depict in Panel (3) the simple subdivision $\left(M^{\prime}, \chi\right)$ of $(M(T ; t, \sigma), \chi)$, and the "fold-up" of $\left(M^{\prime}, \chi\right)$ into $N$ using the folding map $f$ as specified in Lemma 5 (Panel (2)). Enumerating the inner vertices of $(T ; t, \sigma)$ as indicated, the map $\mu$ is given in terms of the labels of the arcs and vertices of $N$ is a TreeNetreconciliation $\mu$ from $(T ; t, \sigma)$ to $N$ and the map $\kappa_{\mu, f}$ represented in terms of the labels of the arcs and vertices of $\left(M^{\prime}, \chi\right)$ is the MUL-reconciliation map $\kappa_{\mu, f}$ from $(T ; t, \sigma)$ to $\left(M^{\prime}, \chi\right)$ as constructed in Section 9. 
extending a directed path $P\left(\rho_{N}, \mu(w)\right)$ of $N$ by a directed path $P(\mu(w), \mu(v))$. Then the choice of $v$ combined with the fact that $f$ is a folding from $M$ to $N$ implies that there exists a path in $M$ from $\rho_{M}$ via $\kappa_{\mu, f}(w)$ to $h_{M}\left(\widetilde{b_{q}}\right)$ where $u_{q}$ is the last but one vertex on $P$ and $b_{q}$ is the arc $\left(u_{q}, \mu(v)\right)$ of $N$. Note that $u_{q}$ must exist since $\mu(v) \neq \mu(w)$. In this case, we put $\kappa_{\mu, f}(v)=h_{M}\left(\widetilde{b_{q}^{u_{q}}}\right)$.

Putting $v=v_{1}$, we can continue with all other children $v_{2}, \ldots, v_{k}$ of $w$. Since we want $\kappa_{\mu, f}$ to be a MUL-reconciliation, we need to ensure that $\kappa_{\mu, f}$ satisfies (M2.i).

Since $\mu$ satisfies Property (R2.i), there must exist children $w_{i}$ and $w_{j}$ of $w$ such that $\mu(w) \in$ $Q_{N}\left(\mu\left(w_{i}\right), \mu\left(w_{j}\right)\right)$. For $l \in\{i, j\}$ put $P_{l}:=P\left(\mu_{w}, z_{l}\right)$ in case $z_{l}$ is a vertex of $N$ and $P_{l}:=$ $P\left(\mu_{w}, h_{N}\left(z_{l}\right)\right)$ in case $z_{l}$ is an arc of $N$. Then since $\mu(w)$ separates $\mu\left(w_{i}\right)$ and $\mu\left(w_{j}\right)$ it follows that regardless of whether or not $\mu\left(w_{i}\right)$ and $\mu\left(w_{j}\right)$ are comparable, we may always choose directed paths $P_{i}:=P\left(\mu_{w}, z_{i}\right)$ and $P_{j}:=P\left(\mu_{w}, \mu\left(z_{j}\right)\right)$ in such a way that the first arc on $P_{i}$ is distinct from the first arc on $P_{j}$.

For each $l \in\{2, \ldots, k\}$, let $P_{l}$ denote a path that is chosen this way. Assuming that $\kappa_{\mu, f}\left(v_{i}\right)$ has already been defined for some $1 \leq i<k$, we then put $\kappa_{\mu, f}\left(v_{i+1}\right)=h_{M}\left(\widetilde{b_{q}}\right)$ where $u_{q}$ is the last vertex on $P_{i+1}$ and $b_{q}$ is the $\operatorname{arc}\left(u_{q}, \mu\left(v_{i+1}\right)\right)$ of $N$.

Case (b): If $t(v)=\mathfrak{s}$ and $t(w)=\mathfrak{d}$, then $\mu(v)$ is a vertex of $N$ and $\mu(w)$ is an arc of $N$. If $h_{N}(\mu(w))=\mu(v)$ then we put $\kappa_{\mu, f}(v)=h_{M}\left(\kappa_{\mu, f}(w)\right)$. So assume that $h_{N}(\mu(w)) \neq \mu(v)$. Consider the directed path $P$ obtained by extending a directed path $P\left(\rho_{N}, h_{N}(\mu(w))\right)$ of $N$ by a directed path $P\left(h_{N}(\mu(w)), \mu(v)\right)$. Then the choice of $v$ combined with the fact that $f$ is a folding of $M$ into $N$ implies that there exists a path from $\rho_{M}$ via $h_{M}\left(\kappa_{\mu, f}(w)\right)$ to $h_{M}\left(\widetilde{b_{q}}\right)$ where $u_{q}$ and $b_{q}$ are as in Case (a). Then we put $\kappa_{\mu, f}(v)=h_{M}\left(\widetilde{b_{q}^{u_{q}}}\right)$.

Case (c): If $t(v)=\mathfrak{d}$ and $t(w)=\mathfrak{s}$, then $\mu(v)$ is an arc of $N$ and $\mu(w)$ is a vertex of $N$. Again, we assume first that none of the children of $w$ have been assigned a value under $\kappa_{\mu, f}$. If $t_{N}(\mu(v))=\mu(w)$ then we define $\kappa_{\mu, f}(v)=\widetilde{\mu(v)^{\mu(w)}}$. So assume $t_{N}(\mu(v)) \neq \mu(w)$. Consider the directed path $P$ obtained by extending a directed directed $P\left(\rho_{N}, \mu(w)\right)$ of $N$ by a directed path $P\left(\mu(w), h_{N}(\mu(v))\right)$. Then the choice of $v$ combined with the fact that $f$ is a folding from $M$ to $N$ implies that there exists a directed path from $\rho_{M}$ via $\kappa_{\mu, f}(w)$ to $h_{M}\left(\widetilde{b_{q}^{u_{q}}}\right)$ where $u_{q}$ is the last but one vertex of $P$ and $b_{q}$ is the $\operatorname{arc}\left(u_{q}, h_{N}(\mu(v))\right)$ of $N$. Then we put $\kappa_{\mu, f}(v)=\widetilde{b_{q}^{u_{q}}}$. Putting again $v=v_{1}$, we can continue with all other children $v_{2}, \ldots, v_{k}$ of $w$ in the same way as in Case (a). Employing analogous arguments, it follows that $\kappa_{\mu, f}$ satisfies (M2.i) for Case (c).

Case (d): If $t(v)=t(w)=\mathfrak{d}$, then both $\mu(v)$ and $\mu(w)$ are arcs of $N$. Consider the directed path $P$ obtained by extending a directed path $P\left(\rho_{N}, h_{N}(\mu(w))\right)$ of $N$ by a directed path $P\left(h_{N}(\mu(w)), h_{N}(\mu(v))\right)$. Then the choice of $v$ combined with the fact that $f$ is a folding from $M$ into $N$ implies that there exists a directed path from $\rho_{M}$ via $t_{N}\left(\kappa_{\mu, f}(w)\right), h_{N}\left(\kappa_{\mu, f}(w)\right)$ and $t_{M}\left(\widetilde{b_{q}^{u_{q}}}\right)$ (note that $t_{M}\left(\widetilde{b_{q}^{u_{q}}}\right)$ and $h_{N}\left(\kappa_{\mu, f}(w)\right)$ might coincide) to $h_{M}\left(\widetilde{b_{q}^{u_{q}}}\right)$ where $u_{q}$ and $b_{q}$ are as in Case (c). Then we put $\kappa_{\mu, f}(v)=\widetilde{b_{q}^{u_{q}}}$.

This completes the definition of $\kappa_{\mu, f}$. Note that $\kappa_{\mu, f}$ can be regarded as a "lifting" of the map $\mu$ along $f$ in the sense discussed in [30, Section 5]. We now show that $\kappa_{\mu, f}$ is indeed a MUL-reconciliation map from $(T ; t, \sigma)$ to $(M, \chi)$.

Proposition 6. Let $(T ; t, \sigma)$ be an event-labeled gene tree, let $N$ be a species network on $\mathbb{S}$, and let $\mu$ be a TreeNet-reconciliation map from $(T ; t, \sigma)$ to $N$. Moreover, let $(M, \chi)$ be a pseudo MULtree that can be folded via a folding map $f=\left(f_{V}, f_{E}\right)$ to $N$. Then, $\kappa_{\mu, f}$ is a MUL-reconciliation map from $(T ; t, \sigma)$ to $(M, \chi)$.

Proof. Let $T=(V, E)$, let $N=(W, F)$ and let $M=(D, U)$. Note that in view of Proposition 2, we may assume w.l.o.g. that $(M, \chi)=\left(U^{*}(N), \chi^{*}\right)$. Thus, $\rho_{N}=\rho_{M}$. 


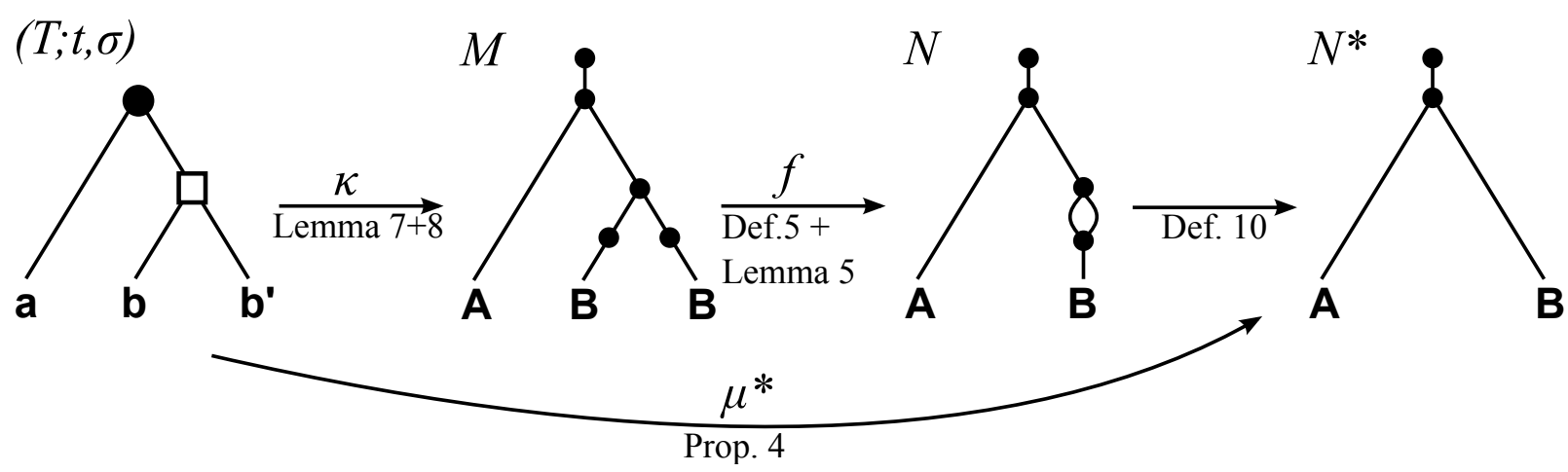

Figure 8: An event-labeled gene tree $(T ; t, \sigma)$ with speciation events $\bullet$ and duplication events $\square$, the simple subdivision $M$ of the MUL-tree $M(T ; t, \sigma)$, the network $N$ constructed in Definition 5 , and the network $N^{*}$ associated to $N$ as specified in Definition 10. Lemma 7 and 8 imply that there is a MUL-tree reconciliation map $\kappa$ from from $(T ; t, \sigma)$ to $M$. As outlined in the proof of Lemma 5 , there is a folding map $f$ from $M$ to $N$. In addition, Proposition 4 implies that there is is a TreeNet-reconciliation map $\mu^{*}$ from $(T ; t, \sigma)$ to $N^{*}$. However, there does not exist a folding map from $M$ to $N^{*}$.

Clearly the map $\kappa_{\mu, f}$ is well-defined. Also, Property (M1) holds in view of $\mu$ satisfying Property (R1).

To see that $\kappa_{\mu, f}$ satisfies Property (M2) assume that $v \in V \backslash L(T)$. If $t(v)=\mathfrak{d}$ then $\mu(v)$ is an arc of $N$. Hence, $\kappa_{\mu, f}(v) \in U$, by the definition of $\kappa_{\mu, f}(v)$. So Property (M2.ii) must hold. If $t(v)=\mathfrak{s}$, then Cases (a) and (c) already imply that Property (M2.i) is satisfied.

To see that $\kappa_{\mu, f}$ satisfies Property (M3), assume that $x, y \in V$ such that $x \prec_{T} y$. We start with establishing Property (M3.i). Assume that $t(x)=t(y)=\mathfrak{d}$. Then $\mu(x)$ and $\mu(y)$ are $\operatorname{arcs}$ of $N$. Since $\mu(x) \preceq_{N} \mu(y)$ as $\mu$ satisfies Property (R3.i) it follows that there exists a directed path $P$ in $N$ from $\rho_{N}$ to $\mu(x)$ that contains $\mu(y)$. Since $M=U^{*}(N)$, the definition of $\kappa_{\mu, f}$ implies that $\kappa_{\mu, f}(x) \preceq_{M} \kappa_{\mu, f}(y)$. Thus, Property (M3.i) must hold.

To see that Property (M3.ii) holds assume that at least one of $t(x)$ and $t(y)$ equals $\mathfrak{s}$. Then $\mu(x) \prec_{N} \mu(y)$ as $\mu$ satisfies Property (R3.ii). We first consider the case that $t(x)=\mathfrak{s}$. Then $\mu(x)$ is a vertex of $N$. Hence, $\mu(x) \preceq_{N} h_{N}(\mu(y))$ in case $\mu(y) \in F$ and $\mu(x) \prec_{N} \mu(y)$ in case $\mu(y) \in W$. In either case, there exists a directed path in $N$ from $\rho_{N}$ to $\mu(x)$ that contains $\mu(y)$. Since $M=U^{*}(N)$, the definition of $\kappa_{\mu, f}$ implies that $\kappa_{\mu, f}(x) \prec \kappa_{\mu, f}(y)$. Thus, Property (M3.ii) holds.

Finally, assume that $t(x)=\mathfrak{d}$. Then $\mu(x)$ is an arc of $N$ and $t(y)=\mathfrak{s}$. Hence, $\mu(y) \in W$. Consequently, there exists a directed path in $N$ from $\rho_{N}$ to $\mu(x)$ that crosses $\mu(y)$. Since $M=$ $U^{*}(N)$, the definition of $\kappa_{\mu, f}$ implies that $\kappa_{\mu, f}(x) \prec \kappa_{\mu, f}(y)$. Thus, Property (M3.ii) must hold in this final case too.

As immediate consequences of Theorems 4, 5 and Proposition 6 we obtain the result that we promised above.

Theorem 7. Suppose $(T ; t, \sigma)$ is an event-labeled gene tree and $N$ is a multi-arc free network. Then $N$ is a species network for $(T ; t, \sigma)$ if and only if $U^{*}(N)$ is a pseudo-MUL-tree for $(T ; t, \sigma)$.

Note that the existence of reconciliations from an event-labeled gene tree to both a MUL-tree $M$ and a network $N^{*}$, does not necessarily imply that there exists a folding map from $M$ to $N^{*}-$ see Fig. 8. This issue is related to the fact that Theorem 7 is stated in terms of $U^{*}(N)$ and not $U(N)$, and that a network $N$ is not always a folding of $U(N)$ (cf. Theorem 4).

\section{Outlook}

In this paper, we have introduced the concept of a reconciliation map between an arbitrary eventlabeled gene tree and a species network, called a TreeNet-reconciliation map. In particular, we 
have shown that for every well-behaved event-labeled gene tree $(T ; t, \sigma)$ there is always a (multiarc free) species network $N$ together with a TreeNet-reconciliation map $\mu$ from $(T ; t, \sigma)$ to $N$ such that $N$ displays all informative triples in $\mathcal{S}(T ; t, \sigma)$. Both the network and the reconciliation map can be constructed in polynomial-time.

These results open up several interesting new avenues for research. For example, although we know that for every event-labeled gene tree $(T ; t, \sigma)$ there is always a species network $N$ for that tree, we do not know much about the computational complexity of determining whether $(T ; t, \sigma)$ can be reconciled with an arbitrary given species network $N$. Clearly, if the unfolded network $U^{*}(N)$ is isomorphic to some subdivision of the MUL-tree $(M(T ; t, \sigma), \chi)$, then we can re-use the latter results based on the MUL-reconciliation map $\kappa$ and the folding $f$ from $(M(T ; t, \sigma), \chi)$ to $N$ to conclude that $N$ is a network for $(T ; t, \sigma)$. However, not all TreeNet-reconciliation maps $\mu$ can be expressed in terms of $\kappa$ and $f$; see Fig. 8. The results and proof techniques in [30, Section 7] may offer an avenue to solving these problems. In this regards it could also be interesting to understand whether the notion of a folding map could be adapted so that it more directly captures the relation between MUL-trees and networks.

In another direction, the structure of the species network obtained from an event-labeled gene tree is heavily dependent on our construction via MUL-trees and foldings, and so it is not clear which properties these networks will enjoy. By way of example, the bi-connected component in the network in Fig. 6 contains two hybrid vertices and it is not clear whether or not a less complex network for the given gene tree may exist (e.g. with fewer hybrid vertices). It would be interesting to investigate properties of the species network, especially how far they are from being minimal under various criteria.

Finally, we have only considered speciation and duplication events. It would be interesting to develop a theory to cope with other events such as horizontal gene transfer. Note that several results have been derived for accommodating gene transfer in reconciliation models between gene trees and species trees, both for unlabeled [22] and event-labeled gene trees [26, 43].

\section{Acknowledgements}

MH would like to thank the School of Computing Sciences, University of East Anglia, and KH and VM would like to thank the Institute of Mathematics and Computer Science, University of Greifswald, for helping to make two visits possible during which this work was conceived and developed.

\section{References}

[1] A M Altenhoff and C. Dessimoz. Phylogenetic and functional assessment of orthologs inference projects and methods. PLoS Comput Biol., 5:e1000262, 2009.

[2] A M Altenhoff, M Gil, G H Gonnet, and C Dessimoz. Inferring hierarchical orthologous groups from orthologous gene pairs. PLoS ONE, 8(1):e53786, 2013.

[3] A M Altenhoff et al. The OMA orthology database in 2015: function predictions, better plant support, synteny view and other improvements. Nucleic Acids Res, 43(D1):D240D249, 2015.

[4] Eric Bapteste, Leo van Iersel, Axel Janke, Scot Kelchner, Steven Kelk, James O McInerney, David A Morrison, Luay Nakhleh, Mike Steel, Leen Stougie, et al. Networks: expanding evolutionary thinking. Trends in Genetics, 29(8):439-441, 2013. 
[5] F Chen, A J Mackey, C J Stoeckert, and D S Roos. OrthoMCL-db: querying a comprehensive multi-species collection of ortholog groups. Nucleic Acids Res, 34(S1):D363-D368, 2006.

[6] Yun Cui, Jesper Jansson, and Wing-Kin Sung. Polynomial-time algorithms for building a consensus mul-tree. Journal of Computational Biology, 19(9):1073-1088, 2012.

[7] Éva Czabarka, PL Erdős, V Johnson, and V Moulton. Generating functions for multi-labeled trees. Discrete Applied Mathematics, 161(1-2):107-117, 2013.

[8] Riccardo Dondi, Nadia El-Mabrouk, and Manuel Lafond. Correction of weighted orthology and paralogy relations-complexity and algorithmic results. In International Workshop on Algorithms in Bioinformatics, pages 121-136. Springer, 2016.

[9] Riccardo Dondi, Nadia El-Mabrouk, and Krister M. Swenson. Gene tree correction for reconciliation and species tree inference: Complexity and algorithms. Journal of Discrete Algorithms, 25:51 - 65, 2014. 23rd Annual Symposium on Combinatorial Pattern Matching.

[10] Riccardo Dondi, Manuel Lafond, and Nadia El-Mabrouk. Approximating the correction of weighted and unweighted orthology and paralogy relations. Algorithms for Molecular Biology, 12(1):4, 2017.

[11] Riccardo Dondi, Giancarlo Mauri, and Italo Zoppis. Orthology correction for gene tree reconstruction: Theoretical and experimental results. Procedia Computer Science, 108:1115 - 1124, 2017. International Conference on Computational Science, ICCS 2017, 12-14 June 2017, Zurich, Switzerland.

[12] Jean-Philippe Doyon, Cedric Chauve, and Sylvie Hamel. Space of gene/species trees reconciliations and parsimonious models. Journal of Computational Biology, 16(10):1399-1418, 2009.

[13] Jean-Philippe Doyon, Vincent Ranwez, Vincent Daubin, and Vincent Berry. Models, algorithms and programs for phylogeny reconciliation. Briefings in bioinformatics, 12(5):392400, 2011.

[14] Jean-Philippe Doyon, Celine Scornavacca, K. Yu. Gorbunov, Gergely J. Szöllősi, Vincent Ranwez, and Vincent Berry. An Efficient Algorithm for Gene/Species Trees Parsimonious Reconciliation with Losses, Duplications and Transfers, pages 93-108. Springer Berlin Heidelberg, Berlin, Heidelberg, 2010.

[15] Oliver Eulenstein, Snehalata Huzurbazar, and David A Liberles. Reconciling phylogenetic trees. Evolution after gene duplication, pages 185-206, 2010.

[16] W M Fitch. Distinguishing homologous from analogous proteins. Syst Zool, 19:99-113, 1970.

[17] Walter M. Fitch. Homology: a personal view on some of the problems. Trends Genet., 16:227-231, 2000.

[18] Nathalie Gontier. Reticulate evolution everywhere. In Reticulate evolution, pages 1-40. Springer, 2015.

[19] Morris Goodman, John Czelusniak, G. William Moore, A. E. Romero-Herrera, and Genji Matsuda. Fitting the gene lineage into its species lineage, a parsimony strategy illustrated by cladograms constructed from globin sequences. Systematic Biology, 28(2):132-163, 1979. 
[20] P. Gorecki and J. Tiuryn. DLS-trees: A model of evolutionary scenarios. Theoretical Computer Science, 359(1):378 - 399, 2006.

[21] WC Thomas Gregg, S Hussain Ather, and Matthew W Hahn. Gene-tree reconciliation with mul-trees to resolve polyploidy events. Systematic biology, 66(6):1007-1018, 2017.

[22] Michael T Hallett and Jens Lagergren. Efficient algorithms for lateral gene transfer problems. In Proceedings of the fifth annual international conference on Computational biology, pages 149-156. ACM, 2001.

[23] Reza Hassanzadeh, Changiz Eslahchi, and Wing-Kin Sung. Do triplets have enough information to construct the multi-labeled phylogenetic tree? PLOS ONE, 9(7):1-10, 072014.

[24] M. Hellmuth, M. Hernandez-Rosales, K. T. Huber, V. Moulton, P. F. Stadler, and N. Wieseke. Orthology relations, symbolic ultrametrics, and cographs. J. Math. Biology, 66(1-2):399420, 2013.

[25] M. Hellmuth and N. Wieseke. From sequence data including orthologs, paralogs, and xenologs to gene and species trees. In P. Pontarotti, editor, Evolutionary Biology: Convergent Evolution, Evolution of Complex Traits, Concepts and Methods, pages 373-392, Cham, 2016. Springer.

[26] Marc Hellmuth. Biologically feasible gene trees, reconciliation maps and informative triples. Algorithms for Molecular Biology, 12(1):23, 2017.

[27] Marc Hellmuth, Peter F Stadler, and Nicolas Wieseke. The mathematics of xenology: Dicographs, symbolic ultrametrics, 2-structures and tree-representable systems of binary relations. Journal of Mathematical Biology, 75(1):199-237, 2017.

[28] M. Hernandez-Rosales, M. Hellmuth, N. Wieseke, K. T. Huber, V. Moulton, and P. F. Stadler. From event-labeled gene trees to species trees. BMC Bioinformatics, 13(Suppl 19):S6, 2012.

[29] K T Huber and V. Moulton. Phylogenetic networks from multi-labelled trees. Journal of Mathematical Biology, 52(5):613-632, 2006.

[30] Katharina T Huber, Vincent Moulton, Mike Steel, and Taoyang Wu. Folding and unfolding phylogenetic trees and networks. Journal of Mathematical Biology, 73(6-7):1761-1780, 2016.

[31] Katharina T Huber, Bengt Oxelman, Martin Lott, and Vincent Moulton. Reconstructing the evolutionary history of polyploids from multilabeled trees. Molecular Biology and Evolution, 23(9):1784-1791, 2006.

[32] Daniel H. Huson, Regula Rupp, and Celine Scornavacca. Phylogenetic Networks: Concepts, Algorithms and Applications. Cambridge University Press, 2010.

[33] Daniel H Huson and Celine Scornavacca. A survey of combinatorial methods for phylogenetic networks. Genome biology and evolution, 3:23-35, 2011.

[34] Manuel Lafond, Riccardo Dondi, and Nadia El-Mabrouk. The link between orthology relations and gene trees: a correction perspective. Algorithms for Molecular Biology, 11(1):1, 2016.

[35] Manuel Lafond and Nadia El-Mabrouk. Orthology and paralogy constraints: satisfiability and consistency. BMC Genomics, 15(6):S12, 2014. 
[36] Manuel Lafond and Nadia El-Mabrouk. Orthology relation and gene tree correction: complexity results. In International Workshop on Algorithms in Bioinformatics, pages 66-79. Springer, 2015.

[37] Manuel Lafond, Krister M Swenson, and Nadia El-Mabrouk. An optimal reconciliation algorithm for gene trees with polytomies. In International Workshop on Algorithms in Bioinformatics, pages 106-122. Springer, 2012.

[38] M Lechner, S Findeiß, L Steiner, M Marz, P F Stadler, and S J Prohaska. Proteinortho: detection of (co-)orthologs in large-scale analysis. BMC Bioinformatics, 12:124, 2011.

[39] Marcus Lechner, Maribel Hernandez-Rosales, D. Doerr, N. Wiesecke, A. Thevenin, J. Stoye, Roland K. Hartmann, Sonja J. Prohaska, and Peter F. Stadler. Orthology detection combining clustering and synteny for very large datasets. PLOS ONE, 9(8):e105015, 082014.

[40] Martin Lott, Andreas Spillner, Katharina T Huber, Anna Petri, Bengt Oxelman, and Vincent Moulton. Inferring polyploid phylogenies from multiply-labeled gene trees. BMC evolutionary biology, 9(1):216, 2009.

[41] B. Ma, M. Li, and L. Zhang. From gene trees to species trees. SIAM Journal on Computing, 30(3):729-752, 2000.

[42] Bin Ma, Ming Li, and Louxin Zhang. From gene trees to species trees. SIAM Journal on Computing, 30(3):729-752, 2000.

[43] Nikolai Nøjgaard, Manuela Geiß, Daniel Merkle, Peter F. Stadler, Nicolas Wieseke, and Marc Hellmuth. Time-consistent reconciliation maps and forbidden time travel. Algorithms for Molecular Biology, 13(1):2, 2018.

[44] RD Page. Genetree: comparing gene and species phylogenies using reconciled trees. Bioinformatics, 14(9):819-820, 1998.

[45] David Posada. Phylogenomics for systematic biology. Systematic biology, 65(3):353-356, 2016.

[46] L Yu Rusin, EV Lyubetskaya, K Yu Gorbunov, and VA Lyubetsky. Reconciliation of gene and species trees. BioMed research international, 2014, 2014.

[47] Celine Scornavacca, Vincent Berry, and Vincent Ranwez. From gene trees to species trees through a supertree approach. In International Conference on Language and Automata Theory and Applications, pages 702-714. Springer, 2009.

[48] Celine Scornavacca, Joan Carles Pons Mayol, and Gabriel Cardona. Fast algorithm for the reconciliation of gene trees and LGT networks. Journal of Theoretical Biology, 418:129 137, 2017.

[49] E.L.L. Sonnhammer and G. Östlund. Inparanoid 8: orthology analysis between 273 proteomes, mostly eukaryotic. Nucleic Acids Research, 43(D1):D234-D239, 2015.

[50] Mike Steel. Phylogeny: discrete and random processes in evolution. SIAM, 2016.

[51] Maureen Stolzer, Han Lai, Minli Xu, Deepa Sathaye, Benjamin Vernot, and Dannie Durand. Inferring duplications, losses, transfers and incomplete lineage sorting with nonbinary species trees. Bioinformatics, 28(18):i409, 2012. 
[52] Gergely J. Szöllősi and Vincent Daubin. Modeling gene family evolution and reconciling phylogenetic discord. In Maria Anisimova, editor, Evolutionary Genomics: Statistical and Computational Methods, Volume 2, pages 29-51, Totowa, NJ, 2012. Humana Press.

[53] Gergely J. Szöllősi, Eric Tannier, Vincent Daubin, and Bastien Boussau. The inference of gene trees with species trees. Systematic Biology, 64(1):e42, 2015.

[54] R L Tatusov, M Y Galperin, D A Natale, and E V Koonin. The COG database: a tool for genome-scale analysis of protein functions and evolution. Nucleic Acids Research, 28(1):33-36, 2000.

[55] Thu-Hien To and Celine Scornavacca. Efficient algorithms for reconciling gene trees and species networks via duplication and loss events. BMC Genomics, 16(10):S6, Oct 2015.

[56] A. Tofigh, M. Hallett, and J. Lagergren. Simultaneous identification of duplications and lateral gene transfers. IEEE/ACM Trans Comput Biol Bioinf, 8, 2011.

[57] K Trachana, T A Larsson, S Powell, W-H Chen, T Doerks, J Muller, and P Bork. Orthology prediction methods: A quality assessment using curated protein families. BioEssays, 33(10):769-780, 2011.

[58] Benjamin Vernot, Maureen Stolzer, Aiton Goldman, and Dannie Durand. Reconciliation with non-binary species trees. Journal of Computational Biology, 15(8):981-1006, 2008. 